\title{
Review
}

\section{Towards ultimate high-power scaling: coherent beam combing of fiber lasers}

\author{
Hossein Fathi ${ }^{1,}{ }^{*}$, Mikko Närhi ${ }^{1}$ and Regina Gumenyuk ${ }^{1}$ \\ ${ }^{1}$ Laboratory of Photonics, Tampere University, Korkeakoulunkatu 3, Tampere 33720, Finland \\ , Finland \\ ${ }^{*}$ Correspondence: h.fathi2500@gmail.com
}

\begin{abstract}
Fiber laser technology has been demonstrated as a versatile and reliable approach for laser source manufacturing with a wide range of applicability in various fields ranging from science to industry. The power/energy scaling of single fiber laser systems has faced several fundamental limitations. To overcome them and to boost the power/energy level even further, combining the output powers of multiple lasers has become the primary approach. Among various combining techniques, the coherent beam combining of fiber amplification channels is the most promising approach, instrumenting ultra-high power/energy lasers with near-diffraction-limited beam quality. This paper provides a comprehensive review of the progress of coherent beam combining for both continuouswave and ultrafast fiber lasers. The concept of coherent beam combining from basic notions to specific details of methods, requirements, and challenges are discussed, along with reporting some practical architectures for both continuous and ultrafast fiber lasers.
\end{abstract}

Keywords: Fiber lasers and amplifiers; pulsed lasers, continuous wave, power scaling; coherent beam combining.

\section{Introduction}

Fiber laser technology has been a topic of extensive research due to its outstanding properties, which are attractive for an enormous number of light-induced applications ranging from science to industry and consequently has advanced rapidly in late years [18]. Fiber laser systems are an appealing alternative to other existing laser sources, owing to their intrinsic merits, including power scaling, excellent beam quality and stability, high quantum efficiency, wide gain bandwidth, superior thermal management, and turn-key operation. These outstanding characteristics lead fiber lasers to superior overall performance and have established fiber lasers as the first choice for versatile laser sources in both continuous wave $(\mathrm{CW})$ and pulsed regimes.

\subsection{Power scaling limitations of fiber lasers and amplifiers:}

Many applications such as advanced material processing and laser particle accelerators are demanding ultra-high power/energy lasers, which accelerate the research on the power scaling of fiber lasers. However, pumping through single clad fibers slowed the progress of power scaling and limited the output power to a few watts. Introducing high brightness laser diodes and the concept of double-cladding fibers have made a revolutionary step in fiber laser technology which resulted in unprecedented output powers/energies $[9,10]$. In the $C W$ regime, these developments led to the realization of a $6 \mathrm{~kW}$ singlemode fiber laser in 2021 [11], and $100 \mathrm{~kW}$ multimode fiber laser systems in 2013, both via diode pumping configuration [12]. In addition, a $20 \mathrm{~kW}$ single-mode fiber laser operating in CW mode via tandem pumping configuration was reported in 2013 [13]. 
Simultaneously with the development of laser systems in the CW regime, a significant step forward in the power/energy of ultrashort fiber lasers has been achieved. This was largely progressed thanks to the invention of chirped pulse amplification (CPA) technique in 1985, which has launched a new leap of the power/energy scaling up for ultrafast pulses [14]. In this regard, records of $1.05 \mathrm{~kW}$ average power in 2013 [15], pulse energy of $1.6 \mathrm{~mJ}$ in 2014 [16], and peak power of 6.2 GW in 2014 [16] for femtosecond pulses have been achieved in a single-amplification channel. However, after these development steps, power scaling of single-mode fiber lasers has faced severe physical limitations, which can be classified into four distinct categories: nonlinear effects (NLE), thermal issues, optical damage, and pump power limitations [17-19]. These four limitations will be discussed in detail in the following sections. Theoretical analysis of the most deleterious thermal and nonlinear power limiting effects of CW fiber lasers, allowed prediction of 28-38 kW for diode pumping and 35-52 kW for tandem pumping with allowance for bend-induced mechanical reliability and modal deformation. The maximum value strongly depends on the amplifier gain [20].

\subsubsection{Nonlinear effects (NLEs):}

Power scaling limitations due to detrimental NLEs are constrained mainly by six effects, including stimulated Brillouin scattering (SBS), stimulated Raman scattering (SRS), self-phase modulation (SPM), self-focusing (SF), four-wave mixing (FWM), and transverse mode instability (TMI) [21-23]. These phenomena are mostly intensity and fiber length dependent and typically become more critical when the laser works in a pulsed regime with high peak power. Decreasing the fiber length and peak intensity are the very first general measures to mitigate the NLEs. As a case in point, pulse stretching in the time domain can reduce the peak power, which is the basis of the CPA technique. Fundamentally, NLEs lead to energy transfer to unwanted wavelengths or fiber modes, which can result in unstable operation. Nonlinear effects arise from the nonlinear response of bound electrons of material to the imposed electromagnetic field. In optical fibers, this response is modeled by a dependence of the electron displacement on the third power of the electric field strength and a third-order nonlinear susceptibility tensor. Second-order nonlinear effects disappear for optical fibers due to centrosymmetricity and higher-order effects are typically negligibly weak. Out of the above NLEs, all but TMI can be interpreted as manifestations of the third-order nonlinearity [21,22]. TMI has its basis on the change of the material refractive index under changes in temperature, i.e. the thermo-optic effect [23]. For high-power fiber lasers and amplifiers in continuous wave $(\mathrm{CW})$ operation, the most prominent limitations arise from SRS, SBS, and TMI. However, in the pulsed regime, the pulse duration and spectral linewidth specify which NLE limits the power/energy scaling.

\section{- $\quad$ Stimulated Raman Scattering effect:}

SRS is a nonlinear scattering process, arising from the interaction of light with laserinduced vibrations of the Raman-active medium, associated with intramolecular bonds. SRS can occur inside the rare-earth-doped fiber laser/amplifiers itself or even in passive delivery fibers, resulting in energy transfer to a frequency-downshifted Stokes wave, typically shifted from the laser wavelength by $13 \mathrm{THz}$ in silica fibers. SRS generates forward and backward light, in which the power level is lower for the latter. The backward light can damage the whole system, especially the seed laser in amplifiers. Moreover, SRS can induce TMI in high power master oscillator power amplifiers (MOPAs) [24,25], which emphasises the importance of suppressing SRS. The critical parameters to evaluate the threshold output power of SRS are the effective length of the optical fiber and the effective area of the mode. Various techniques for suppressing SRS have been investigated, among which decreasing the fiber length and increasing the fiber core size are the main ones [2633]. Raman gain coefficient in fibers is typically on the order of $10^{-13} \mathrm{~m} / \mathrm{W}$, which is nearly three orders of magnitude smaller than the Brillouin gain coefficient discussed next. Therefore, SRS is more prominent in pulsed systems, however, can also occur in kW-level CW systems. 
- Stimulated Brillouin Scattering effect:

SBS is a nonlinear process that arises from the interaction between optical photons with acoustic phonons and occurs essentially in propagating/amplifying of narrow bandwidth (CW) signals through a nonlinear medium. This detrimental effect leads to an energy transfer from signal to frequency-downshifted light in the backward direction that is typically shifted by $10-20 \mathrm{GHz}$ in silica fibers. SBS gain coefficient in silica fibers is on the order of $5^{-11} \mathrm{~m} / \mathrm{W}$ and it has the lowest power threshold among the NLEs in single-frequency CW fiber amplifiers. The main approach to mitigating the SBS is broadening the spectral linewidth via external phase modulation [34,35]. To realize the SBS suppression, the change in phase modulation should be less than the phonon lifetime. This is also why SBS does not typically occur for pulsed systems with pulse durations below the $\sim 10$ ns phonon lifetime. Numerous theoretical and experimental research on mitigating the SBS effect in fiber lasers and amplifiers has been conducted to prevent instability and physical damage to the laser system [36-53]. As a case in point, in 2020, Wang et al. demonstrated a $2.5 \mathrm{~kW}$ narrow linewidth linearly polarized MOPA by efficient suppressing of SBS-induced self-pulsing (SP) via implementing cascade phase modulation [54]. In the same year, they presented the first $3 \mathrm{~kW}$ all-fiber narrow linewidth linearly polarized MOPA via a bi-directional pumping scheme [55]. In the case of non-polarized MOPA, in 2019, a $3.7 \mathrm{~kW}$ monolithic narrow-linewidth single-mode fiber amplifier was reported by suppressing the NLEs [56].

- $\quad$ Self-phase modulation effect:

SPM, which is mainly acute for pulsed lasers, refers to a self-induced nonlinear phase shifting process, which arises from the intensity dependence of the refractive index $[21,57,58]$. This effect causes spectral broadening, which depends on the optical pulse shape [57,59]. SPM is a severe limiting factor for the power/energy scaling of ultrashort pulses by the coherent beam combining (CBC) configuration, since the phase-locking loop may not correct the phase shift induced by SPM, leading to degradation of the temporal coherence and combining efficiency.

- Self-focusing effect:

Among the NLEs, SF is responsible for the ultimate peak-power limitation in the ultrafast fiber amplifiers $[8,60]$. SF is caused by the nonlinear refractive index (Kerr effect) resulting in a radially varying refractive index following laser transverse mode intensity profile. This radially changing refractive index works as an effective lens leading to power concentration in a tiny spot and damaging the fiber core. In contrast to SPM, which is the temporal dependence of the intensity-induced phase shift, SF is the transverse dependence of the phase shift. For fiber laser operating in the fundamental transverse mode, SF limits the achievable peak power from fiber lasers to $4-6 \mathrm{MW}$ at around $1 \mu \mathrm{m}$, which corresponds to a pulse energy of $\approx 10 \mathrm{~mJ}$ for 2 ns pulses [60-62]. The SF threshold not only depends on the pulse peak power, but also the polarization state of the laser beam [6163]. In 2009, Schimpf et al. experimentally demonstrated that circular polarization reduces the detrimental impact of the Kerr-nonlinearity by a factor of 2/3 relative to linear polarization, therefore increasing the SP threshold [62].

- Four-wave mixing effect:

FWM refers to a process involving four photons in total: two or three photons are annihilated to create two or one photons at new frequencies. The frequencies are defined by the energy conservation principle. It is a phase-sensitive process which arises only if the phase-matching condition is satisfied. Compared to single-mode fibers at normal dispersion regime wavelengths below $1.3 \mu \mathrm{m}$, multi-mode (MM) fibers are more sensitive to the FWM. Indeed, the phase-matching condition in MM fibers can easily be satisfied, because different frequencies can propagate in different modes with the appropriate group velocity. So, in high power fiber lasers which are based on LMA fibers, FWM can arise when the phase-matching condition is satisfied between the fundamental and high-order 
transverse modes [64]. It is worth mentioning that the phase-matching condition can also be met in anomalous dispersion regime fibers, which is to be considered when working with Erbium and Thulium doped fiber amplifiers.

- $\quad$ Transverse mode instability effect:

TMI is the most prominent power-scaling limitation responsible for beam quality degradation which is the first representative result of thermally-induced non-linear effects in optical fibers $[23,65-71]$. This threshold-like behavior refers to the energy transfer between the fundamental transverse mode and one or more high-order transverse modes, leading to temporal fluctuations of the output power on the $\mathrm{kHz}$ frequency scale. The reason is that high power single-mode fiber lasers and amplifiers, which need the LMA fibers in their gain medium, are designed based on imposing loss for high order modes. Therefore, any energy transferred to the high order modes, converts to loss, leading to the output power fluctuations. Although this effect was reported in 2011, rapid development has been achieved in this field. Consequently, several studies have been reported on the passive and active methods to mitigate the TMI [23]. For example, in 2021, by effective mitigation of the TMI, a $6 \mathrm{~kW}$ single-mode monolithic fiber laser was demonstrated by Yang et al. [11]. By analyzing the thermally induced mode distortion, Ke et al. reported that the highest achievable output power with near-diffraction-limited beam quality for Ytterbium-doped fiber lasers (YDFLs) could be 67-97 kW, depending on the power distribution in the fundamental mode and the V-parameter of the fibers [72]. However, in 2019, by applying the TMI effect on analyzing the power scaling limitations, Zervas corrected the upper limits to $28-38 \mathrm{~kW}$ for diode pumping and $35-52 \mathrm{~kW}$ for tandem pumping schemes [20].

In conclusion of NLEs, it should be highlighted that for broadband fiber laser and amplifiers, SRS and TMI define typically the upper limit for power scaling. In contrast, for high power narrow bandwidth signals, SRS, SBS, and TMI restrict the power scaling [73].

\subsubsection{Thermal issues:}

The most prominent thermal effects which hinder the power/energy scaling are thermal fracture, melting of the core, and thermal lensing, which are discussed in detail in $[17,74,75]$. Quantum defect (QD) is responsible for the localized heat generation in active fibers, which leads to thermal degradation of both the fiber core and the protective polymer layer. In [74], a comprehensive study of thermal limits to power scaling of doubleclad silica fiber laser was carried out, in which the maximum extractable power per unit length was calculated for each thermal effect [74]. Minimizing the heat deposition in the doped core was determined as the most critical point to overcome these limits. To superior power handling and thermal management, they suggested using the large fiber cladding diameters or holey core fiber for better heat dissipation and avoidance of using low thermal conductivity polymers as cladding layers at high powers.

\subsubsection{Optical damage:}

It refers to the damage of optical components induced by high-intensity lasers. In optical fibers, laser-induced damage is most likely to happen to the end facets by highintensity pulses [76-78]. The laser damage threshold (LTD) for the optical surface is lower compared to the bulk materials. For optical fibers, LTD determines the laser power/energy which an optical fiber can handle, and above which damage is more likely to occur. In this regard, for fiber lasers, utilizing components such as mode field adapters, combiners, and end-caps (at the end of fiber output) can decrease the peak intensity, and consequently increase the surface damage threshold.

\subsubsection{Pumping limitations:}

The amount of injected pump power and consequently absorbed pump power determines an important upper limit for power scaling of single-mode lasers, characterised by pump brightness and fiber specifications. The brightness of laser diodes, fiber cladding aperture $\left(N A_{c l}\right)$, and cladding diameter $\left(r_{c l}\right)$ define how much power can be injected into 
the fiber. Moreover, the pump absorption relies on the core diameter $\left(r_{\text {core }}\right)$ and doping concentration of active ions. Therefore, the larger $r_{\text {core }}$, the higher pump absorption can be reached. However, maintaining the single-mode operation in large $r_{\text {core }}$ would only be possible if the core numerical aperture $\left(N A_{\text {core }}\right)$ decreases. Realizing high brightness pump along with the low NA fibers have been faced fabrication challenges [9,79-81]. Furthermore, due to the photodarkening effect [82], the amount of doping concentration is limited, affecting the pump absorption level. Nevertheless, with technology development, there has been a significant improvement to overcome this limitation, especially with emerging high brightness fiber-coupled laser diodes and progress in fabricating low NA fibers $[80,81,83,84]$.

In conclusion of power scaling limitations, the highest achieved output powers for YDFLs have been reported as $6 \mathrm{~kW}$ with a beam quality $\mathrm{M}^{2}$ factor of $\sim 1.3$ [11], and 10.6 $\mathrm{kW}$ with the beam quality $\mathrm{M}^{2}$ factor of $\sim 2$ [85]. Even if the technical challenges for fabricating high power YDFLs could be overcome, the highest theoretical achievable output power through diode pumping is calculated as $28-38 \mathrm{~kW}$ via a diode pumping scheme [20]. Therefore the need for new power scaling approaches beyond the fundamental limitation has been recognized and is an active field of research.

\subsection{Methods for power scaling:}

There are two main approaches to boost the achievable output power of fiber lasers, including tandem pumping (TP) and beam combining, which will be discussed in the following sections.

\subsubsection{Tandem pumping}

Tandem pumping refers to a technique in which high brightness lasers are implemented as pumping sources. Owing to its unique potential for power scaling, it has been achieved great attention in the last decade [19,86-95]. In 2010, IPG photonics corp. reported the first $10 \mathrm{~kW}$ level single-mode fiber laser via TP scheme by utilizing forty-seven $270 \mathrm{~W}$ YDFLs operating at $1018 \mathrm{~nm}$ as pumping sources [96]. Following that, in 2012, they reported the fabrication of $17 \mathrm{~kW}$ using the same sources at $1018 \mathrm{~nm}$ [97], which then improved to $20 \mathrm{~kW}$ in 2013 [13].

This technique provides several outstanding advantages, mitigating the power scaling limitations such as thermal issues, TMI, and pump power limitations $[86,98,99]$. In the $\mathrm{TP}$ technique, lasers at the wavelength of 1010-1030 $\mathrm{nm}$ are typically utilized as pumping sources, among which $1018 \mathrm{~nm}$ is introduced as an appropriate candidate in this range. By closing the pump wavelength to the laser wavelength, the amount of quantum defect responsible for the thermal load can be decreased by almost half. Furthermore, more pump power can be injected into the main gain fiber due to implementing the high brightness pump sources. However, the most prominent advantage of TP is that this method can control the undesired excess gain for high-order modes in large-core fibers [86]. This leads to the mitigation of unwanted effects, caused by the building-up of power in high order modes. As a case in point, TP scheme reduces the risk of self-pulsing induced by high order modes [86,100]. Last but not least, TP has an enormous potential against photodarkening (PD); for instance, PD in pumping with $1045 \mathrm{~nm}$ is 1 million times smaller than pumping with $910 \mathrm{~nm}$ [86]. Nevertheless, the most prominent drawback of TP configuration is its need for a long length of the gain fiber, typically $30-40 \mathrm{~m}$, due to the lower pump absorption via pumping with short wavelengths (such as $1018 \mathrm{~nm}$ ). Herein arise the severe limitations for power scaling owing to the nonlinear scattering processes such as SRS. Recently, Lim et al. have presented a high absorption LMA fiber, $27 \mathrm{~dB} / \mathrm{m}$ at $976 \mathrm{~nm}$, simultaneously with a low NA of 0.04 [81]. This has provided the possibility for reducing the gain fiber length from $30-40 \mathrm{~m}$ to below $15 \mathrm{~m}$.

However, generating stable high-power laser sources at $1018 \mathrm{~nm}$ is demanding due to the small net gain and high amplified spontaneous emission (ASE) at the range of 1030$1035 \mathrm{~nm}$. Indeed, in alumosilicate fibers, laser operation at $1018 \mathrm{~nm}$ needs higher 
population inversion than laser operation at 1060-1080 nm, leading to emerging ASE and spurious oscillations. Herein, unwanted fierce gain competition occurs between ASE and laser at $1018 \mathrm{~nm}$. As a result of ASE, parasitic lasing and self-pulsing can cause physical damage to the systems. To suppress these deleterious effects, shortening the fiber length and using the high core/clad diameter ratio fibers are utilized. There has been ongoing research on producing high power $1018 \mathrm{~nm}$ fiber laser with diffraction-limited beam quality, to date, the highest output power of the YDFLs operating at $1018 \mathrm{~nm}$ fiber laser has been reported by Yan et al., as $1150 \mathrm{~W}$ [89,91-95]. Although the highest record for achievable output power via TP scheme is predicted to be $52 \mathrm{~kW}[18]$, to date it has reached 20 $\mathrm{kW}[13]$.

\subsubsection{Beam combining}

Based on the above discussion, power/energy scaling in single fiber lasers has faced severe physical limitations, which hinder obtaining the high-power lasers with high beam quality and stability. Hence, beam combining of multiple lasers have always been the first measure to increase the power/energy of laser systems beyond the achievable output power from a single laser. Laser beam combining provides an impactful platform for the realization of a single high-power beam from multiple low-power lasers. A combination of individual lasers can be achieved both coherently and incoherently, which will be discussed in detail in the following sections.

This paper will review the beam combination methods and focus on the coherent beam combining (CBC) of fiber lasers in detail. There are excellent separate reviews on CBC of fiber lasers, for both continuous and pulsed lasers [6,101-110]. However, this paper gives a deep insight into the $\mathrm{CBC}$ concept from basic notions to more specific details of methods, requirements, and challenges for both continuous and ultrafast fiber lasers. Additionally, the review highlights the various state-of-the-art CBC architectures and provides with the guideline for some practical aspects of developing new systems. Figure 1 provides a topic map of the laser beam combining, with the main focus on the coherent beam combining approach.

The paper is organized as follows Chapter 2 reviews the incoherent beam combining technique. Chapter 3 describes various techniques in the coherent beam combining approach. In Chapter 4 we focus on the coherent beam combining for ultrafast fiber lasers. Chapter 5 discusses the methods for power scaling of CW lasers by means of beam combining. And the Conclusions chapter summarizes the main aspects of the article.

\section{Incoherent beam combining}

Incoherent beam combining can be classified into three distinct groups, including side-by-side beam combining, combining via passive components and spectral beam combining (SBC).

\subsection{Side-by-side beam combining}

In this scheme, multiple lasers are positioned next to each other, in an array (Figure 1 ), in which there is no control on the phase and spectral relation of the individual lasers, even lasers can work in a different wavelength. The most prominent example of this method is the combination of diode laser arrays, in one or two dimensions. Since this method does not require the narrow laser linewidths or phase/polarization control, it is much easier to implement compared with CBC or SBC. Indeed, it is achieved by overlapping multiple laser beams using beam directors. With some considerations such as expanding the initial beam size and pointing of each beam (controlling the beams overlap with steering mirror, adaptive tip-tilt control, to minimize the turbulence effects), this method can be applied in directed-energy applications [111,112]. In 2015, by utilizing beam directors consisting of individually controlled steering mirrors, four fiber lasers with a total power of $5 \mathrm{~kW}$ were directed to a target $3.2 \mathrm{~km}$ away [112]. Moreover, in 2014, an incoherent combined $30 \mathrm{~kW}$ fiber laser system, consisting of six $5.5 \mathrm{~kW}$ fiber lasers 
directed with different mirrors through a single telescope was demonstrated as a laser weapon system [113].

\subsection{Passive components}

In this approach, by utilizing passive components, multiple lasers will be combined without any control on the phase or polarization of the beams. The most conventional elements implemented in this technique are polarization beam splitters (PBS) and fiberbased combiners (Figure 1). In combination with PBS, two laser beams with orthogonal polarization are combined, resulting in a combined beam with a random polarization state due to the phase fluctuations. This technique is inherently limited to two laser beams. If the phase of initial beams is locked, the polarization of the combined beam can be fixed, and consequently, further combination in cascade scheme would be possible $[108,114]$.

There has been a great development in the passive fiber-based component during the last decade. In 2013, IPG corp. reported a $100 \mathrm{~kW}$ fiber laser by utilizing fused fiber combiners, with 35.4\% wall-plug power consumption efficiency [12]. They implemented 90 fiber lasers with an output power of $1.2 \mathrm{~kW}$, coupled to six $19 \times 1$ combiners. Then, using the main $7 \times 1$ combiner, they achieved $100 \mathrm{~kW}$ laser from fiber with $300 \mu \mathrm{m}$ core diameter with the beam parameter product of $16 \mathrm{~mm} \times$ mrad. In 2018, Lie et al. demonstrated a 14 $\mathrm{kW}$ combined laser beam via an all-fiber structure signal combiner with the beam quality of 5.37 and $98.5 \%$ combining efficiency [115].

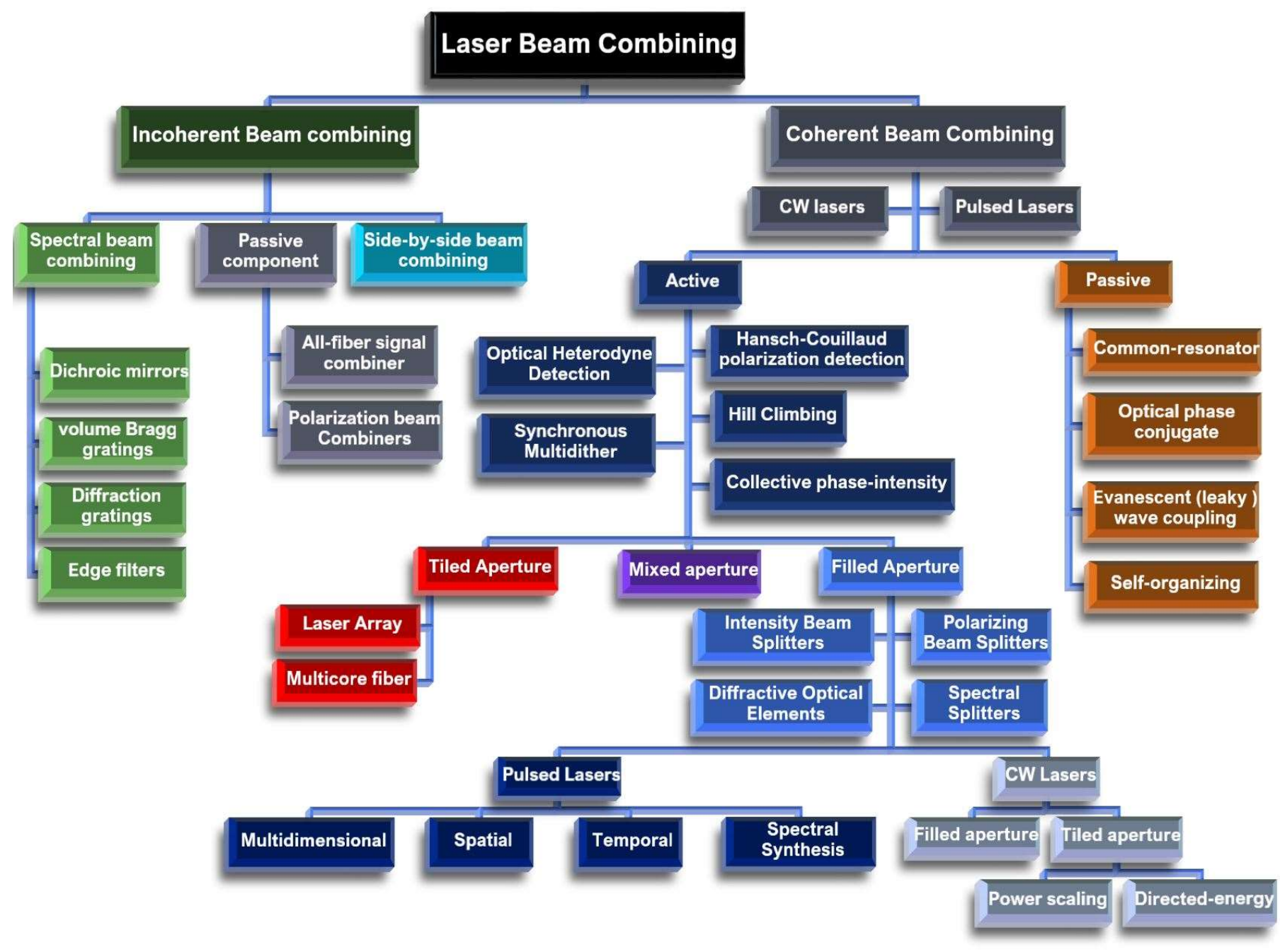

Figure 1. Topic map of the laser beam combining. 


\subsection{Spectral beam combining ( $S B C$ )}

This approach refers to a combining technique in which multiple narrow linewidth laser beams with slightly different wavelengths are combined into a single beam spot with spatially bright diffraction-limited beam quality [116-120]. In this method, the combination will be obtained by utilizing two approaches: (1) dispersive optical elements such as diffractive grating and prisms and (2) with wavelength-dependent transmission elements such as volume Bragg gratings or dichroic mirrors and filters (see Figure 2). SBC has an inherent potential to increase power and brightness at the same time. In the SBC technique, the brightness of the combined beam scales with the number of laser beams. In 2011, Wirth et al. demonstrated an $8.2 \mathrm{~kW}$ laser through spectrally combining four narrowlinewidth fiber amplifiers [121]. In this regard, narrow-band laser sources greatly impact the quality of the combined laser beam.

However, high power narrowband fiber lasers have faced SBS limitation. Implementing all-fiber narrowband ASE sources is an excellent approach to surpass the SBS limitation due to the lack of coherent longitudinal modes of the ASE source. In this regard, in 2016, a $10.8 \mathrm{~kW}$ spectrally combined laser system was demonstrated by utilizing eight SBS-free sources with a combining efficiency of $94 \%$ [122-124]. The combining element implemented in this work was reflective diffraction grating, requiring more space for alignment compared to the transmitting diffraction gratings. In this regard, in 2019, Zheng et al. demonstrated a $10.6 \mathrm{~kW}$ spectrally combined system by utilizing two transmission gratings as a combining system [125]. Moreover, using an edge filter, in a filter-based SBC system, Chen et al. managed to achieve $10.12 \mathrm{~kW}$ with the combination of two $5 \mathrm{~kW}$-level broad-linewidth fiber lasers [126]. There have been great developments in the field of SBC, in which laser systems at the level of few $\mathrm{kW}$ to tens of $\mathrm{kW}$ have been realized $[121,124,126-131]$. As a case in point, in 2016, Honea et al. reported a $30 \mathrm{~kW}$ single laser beam with the beam quality of $M^{2}=1.6 \times 1.8$, realized by SBC of 96 individual fiber lasers, which later improved to $60 \mathrm{~kW}$ in 2017 [132,133].

\section{Coherent beam combining (CBC)}

CBC is referred to as the technique of power scaling by combining multiple laser amplifiers, which are seeded by a common source, into a single high-power beam with maintaining the beam quality [134]. In addition to spatial properties, CBC preserves the spectral properties of the lasers. In this technique, as its name implies, there is a phase relation between laser amplifiers, so the parallel amplifiers are operating effectively as a single laser. The primary notion is that a seed laser split up into several replicas ( $\mathrm{N}$ channels) and amplifying them to the highest possible power/energy through the amplifier sections, and then combined them to a high brightness single beam. To realize coherent beam combining, the phase of each channel should be locked via either passive or active mechanisms.

In 1970, the first demonstration of CBC was realized by combining two semiconductor oscillators [135]. The first implementation of all-fiber CBC of CW fiber lasers was reported by Kozlov et al. in 1999 [136], and nine years later, in 2008, Cheung et al. realized $\mathrm{CBC}$ of pulsed fiber laser array [137]. Due to the enormous potential of the CBC technique for power scaling, it has been extensively developed in recent years and the record of 100 $\mathrm{kW}$ using seven 15-kW Slab MOPAs is already achieved [138]. This paper is focused on fiber lasers combining as one of the most perspective alternatives to produce state-of-theart ultra-high power lasers with excellent laser properties. 


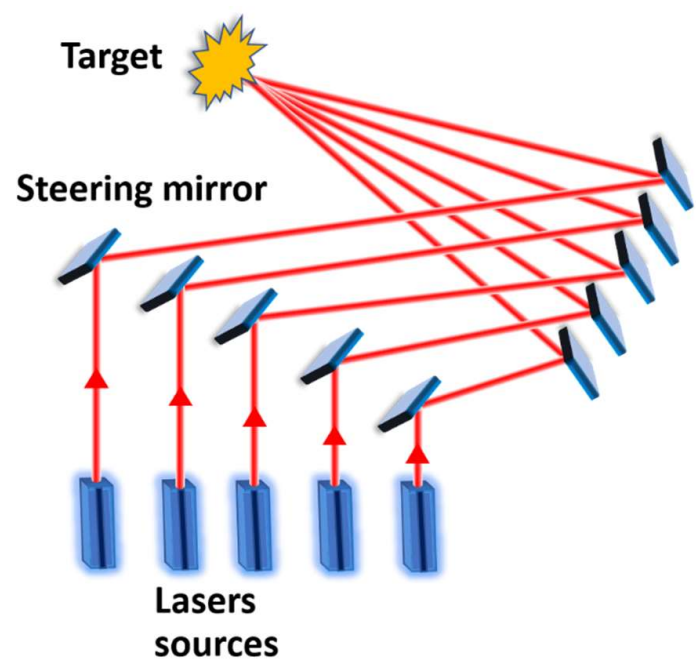

(a)

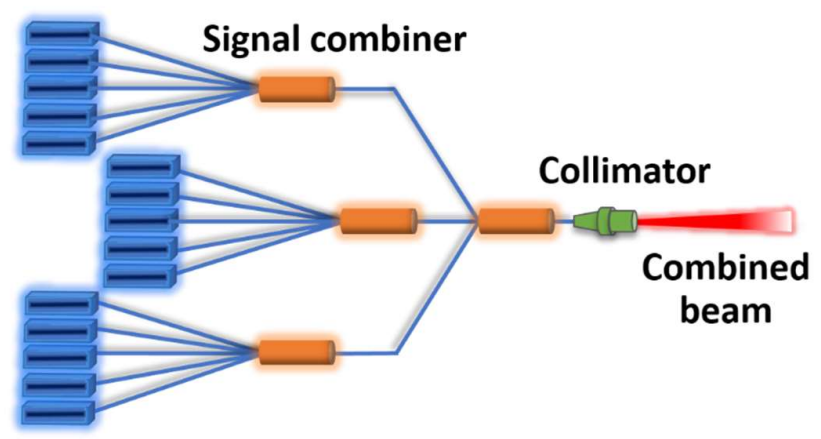

Laser sources

(b)

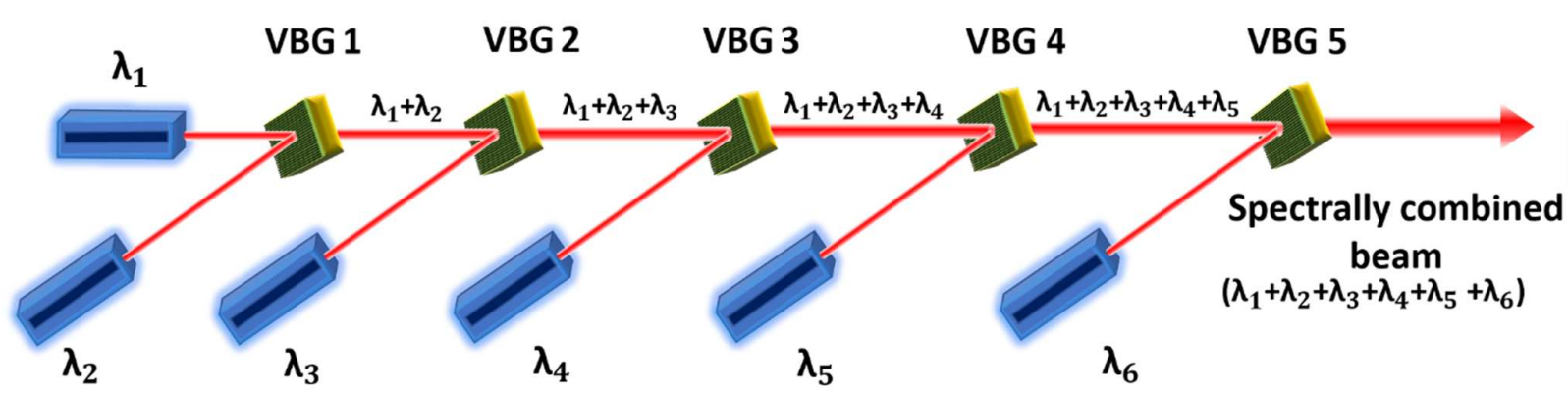

(c)

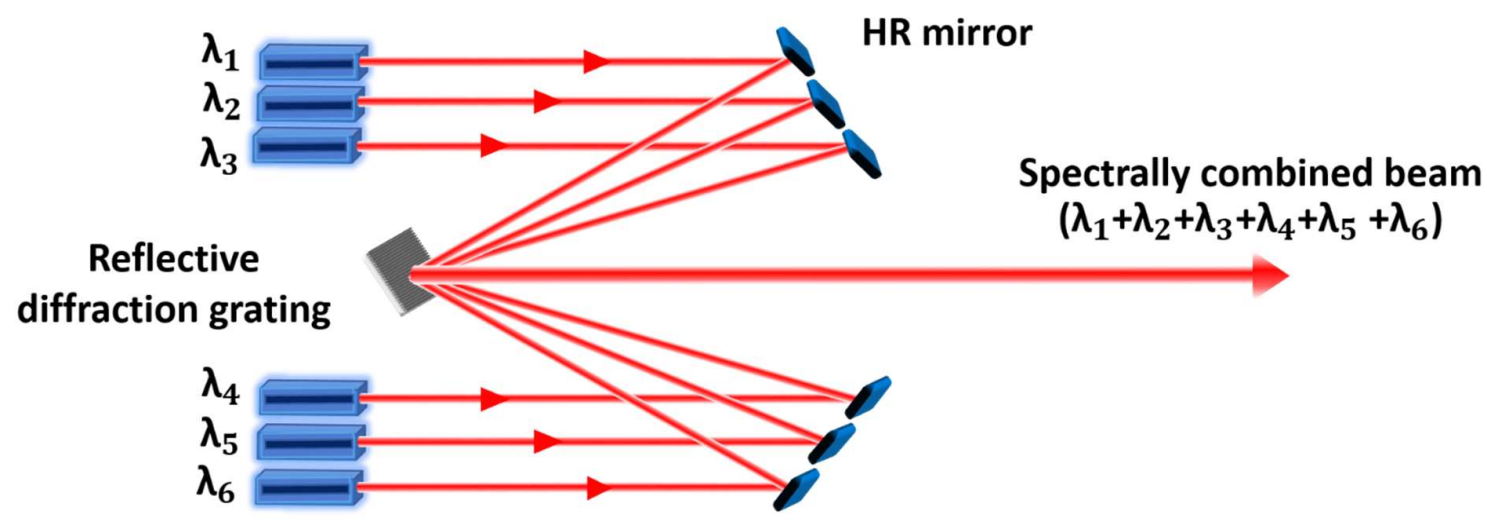

(d)

Figure 2. Schematic representation of four main methods of incoherent beam combining; a) side-by-side beam combining, b) beam combining using all-fiber passive signal components, c) SBC with volume Bragg gratings (VBG) as a wavelength-dependent transmission element, and d) SBC with reflection diffraction grating as a dispersive optical element.

There are two criteria for evaluating the performance of a laser combining system, which are combining efficiency $(\eta)$ and the beam quality $\left(\mathrm{M}^{2}\right)$ of the output laser beam. Combining efficiency is typically calculated by dividing the power of the combined beam by the sum of the output power of amplified laser beams. However, for CBC systems, two key metrics are employed for assessing the function of $\mathrm{CBC}$, which are brightness (B) and Strehl ratio (S). The brightness of each optical beam, which considers the output power and $\mathrm{BQ}$ of the beam $\left(M^{2}\right.$ factor), can be defined as: 


$$
B=\frac{C P}{\lambda^{2}\left(M^{2}\right)^{2}}
$$

where $\mathrm{P}$ is the output power, $\mathrm{C}$ is a constant defined by the beam shape (e.g., $\mathrm{C}=1$ for the Gaussian beam) and $\lambda$ is the laser wavelength. In CBC of $N$ laser amplifiers, the brightness of the combined beam scales by a factor of $\eta \mathrm{N}$, compared to the brightness of a single laser beam. In an ideal case, in which $\eta=1$, the brightness of a coherently combined beam is $N$ times larger than the brightness of an incoherently combined beam in the same combining structure. Indeed, the brightness of the incoherently combined laser amplifiers at the best state is equal to the brightness of a single laser beam [105]. Herein lies the confusion about the brightness enhancement in the CBC systems. In some texts, it is stated that the brightness in ideal CBC will increase by the factor of $\mathrm{N}^{2}$ compared to the brightness of a single laser beam. However, in reality, it increases by a factor of $N$ compared to the brightness of a single laser beam (before implementing them in the combining system). Indeed, in an ideal case, the on-axis far-field intensity increases as $\mathrm{N}^{2}$. Various definitions of comparative parameters, such as the output power of single lasers and emitting aperture, cause confusion regarding the brightness enhancement in CBC systems. For example, two single lasers (with a diameter of $\mathrm{D}$, power of $\mathrm{P}$ and brightness of B) is combined by CBC using a 50:50 intensity beam splitter (IBS). In this combination, there is no change in aperture sizes, but the power of the combined beam will increase to $2 \mathrm{P}$ (in a full working array). Consequently, the brightness will increase to $2 \mathrm{~B}$ at the output port of the IBS, indicating $N$ times enhancement of B. However, if one laser is turned off and only the power of another single laser (after passing the IBS) is considered, the final power of the system will be $\mathrm{P} / 2$ in the output port. Now, if this power is compared to the power of the coherently combined beam, the $\mathrm{B}$ value is increased by a factor of $\mathrm{N}^{2}$.

The next metric for assessing the function of $\mathrm{CBC}$ systems is the Strehl ratio parameter, which has a value between 0 and 1 . It is calculated by dividing the peak intensity in the central lobe of the combined beam by the peak intensity of an ideal phase-locked combined beam. The Strehl ratio is not only sensitive to the phase-locking operation, but also to the laser array aperture fill factor (FF) of combining geometry. There are two wellknown definitions for $\mathrm{FF}$, the first is defined as the ratio of emitting apertures area to the total area occupied by lasers cell, and the second is defined as the ratio of the beam diameter to the central distance of the adjacent beams. In this paper, the former definition is used.

There are several important requirements for realizing an ideal $\mathrm{CBC}$, including the phase correlation between laser beams (phase locking), equal optical path, equal laser power in each channel, same polarization, equal beam size, and spatial co-aligning [139]. Indeed, the individual amplifier channels need to be identical for realizing the maximum combining efficiency.

Figure 3 provides a general schematic illustration of $\mathrm{CBC}$. The $\mathrm{CBC}$ systems typically involve five principal parts: i) the geometry of splitting/combining, ii) laser sources and amplifying section, iii) phase-locking system, iv) optical path difference (OPD) control, and v) number of channels. All of them will be discussed in detail in this section with the emphasis both on the concept and operation principles.

\subsection{The geometry of combining/splitting in space and time}

By 2020, the CBC geometry was classified into two broad categories: tiled aperture and filled aperture geometries [140]. However, Prossotowicz et al. has recently introduced a new concept of mixed-aperture geometry [141].

\subsubsection{Tiled aperture (TA)}

In this scheme, amplified laser beams are positioned next to each other, side-by-side in an array, with the largest possible array filling factor (the minimum gap between individual laser beams) [142]. The laser beams are co-aligned and consequently, the beams 


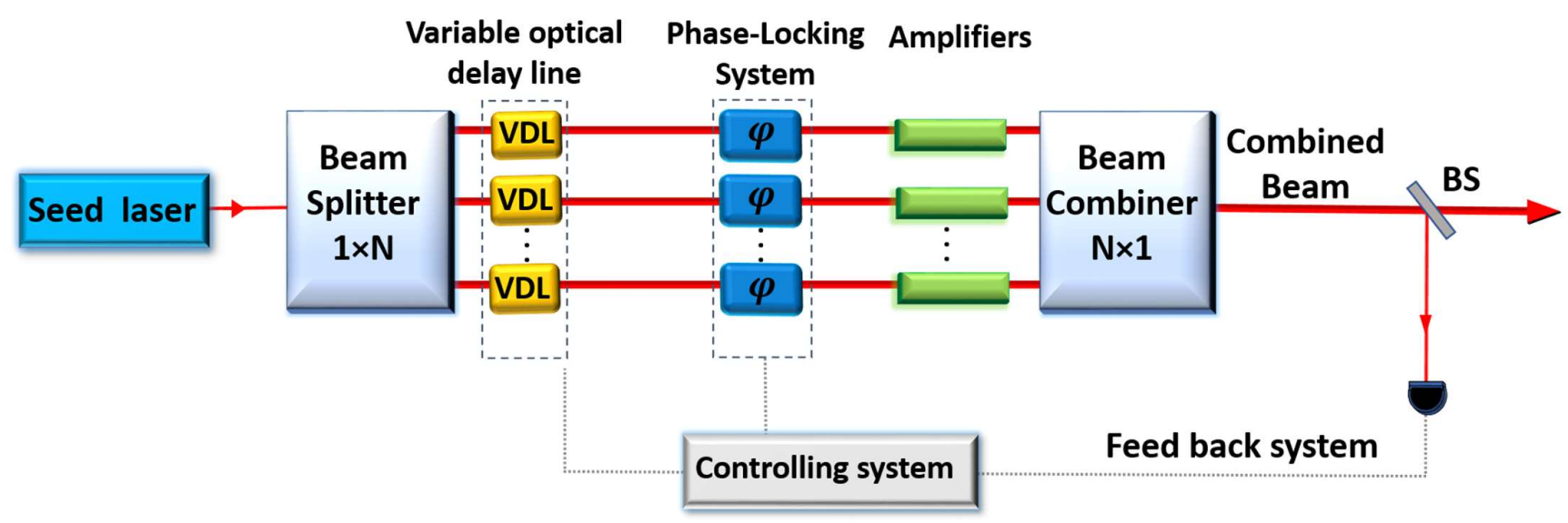

Figure 3. Schematic of coherent beam combining system.

interfere only in the far-field, indicating no beam combining element is required in this approach. Figure 4 shows the far-field intensity patterns of the coherent combination of seven beams in tiled-aperture geometry at different distances, simulated in MATLAB. The laser beams are positioned in a hexagonal pattern with a sub-aperture fill factor (ratio of the beam diameter to the collimator diameter) of $91.6 \%$, and the laser array aperture fill factor of $\mathrm{FF}=76 \%$. It is worth mentioning that in $\mathrm{CBC}$ through tiled-aperture geometry the diameter of the main lobe in the far-field intensity pattern would be smaller than the diameter of a single beam after propagation in the far-field.

This architecture was first utilized in 1982 by Scifres et al. for combining semiconductor lasers [143]. The maximum reported power of coherently beam combined lasers has been demonstrated by a combination of seven $15 \mathrm{~kW}$ slab lasers via laser array architecture, realizing the first $100 \mathrm{~kW}$ coherently combined beam. This scheme is simple to operate and is suitable for a large array of laser beams since it is not limited by power handling issues of combining elements.

The approach is highly sensitive to the physical arrangement of the laser beams in the near-field. It affects the combining efficiency through the fill factor parameter, combining beam symmetry and periodicity of the energy distribution in the far-field. Laser beams can be positioned in different patterns such as square, hexagonal and ring, however, the hexagonal pattern provides the closest possible packing (maximum fill factor), leading to the highest combining efficiency. Moreover, the filling factor affects the phase

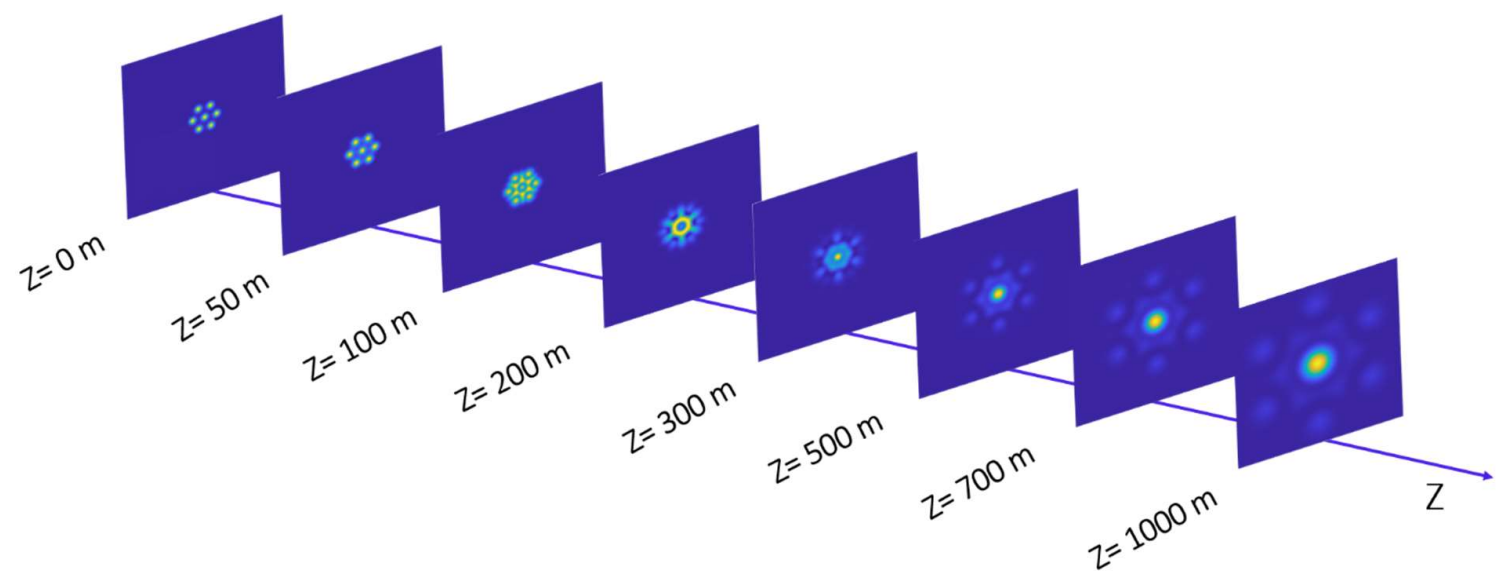

Figure 4. Intensity patterns of the coherent combination of seven beams in tiled-apperture geometry at different distances. The beam profiles are shown in the same frame scale. 
root-mean-square (RMS), which is more acute when the number of combined beams is small [144]. In an ideal case, with the maximum filling factor of $\approx 1$, if the phase of each laser array is locked to each other in the far-field, the effective coherent combination will realize as a single spatial lobe. However, in the case of $\mathrm{FF}<1$, which indicates the nonuniformity in the intensity of the emitting aperture in the near field (due to the gap between the laser beams), a fraction of total power appears in the sidelobes. So, the combining efficiency, which corresponds to how much of the total power is concentrated in the central lobe, will decrease. Indeed, in the tiled aperture combining geometry, the combining efficiency is inherently limited due to the existence of sidelobes, resulting in beam quality degradation. This is known as a significant drawback of tiled aperture combining geometry. Figure 5 clearly shows the impact of the fill factor on the combining efficiency by comparing the far-field intensity of 3 setups with different fill factors ( $\mathrm{FF}=76 \%, 52 \%$ and $39 \%$ ). As can be seen in this figure, by decreasing the FF, the energy distribution is remarkably changed, and energy is mostly transformed from the main lobe to the side lobes. It is reported that the combining efficiency of multiple Gaussian laser beams via tiled aperture geometry at the ideal case is theoretically limited to 76\% [145]. The highest achieved efficiency for CBC of pulsed lasers has been reported as $50 \%$ by a coherent combination of 19 femtosecond lasers with residual phase errors lower than N60 RMS [146]. In the CW regime, the efficiency of $58 \%$ has been achieved by CBC of eight $C W$ fiber amplifiers with a total output power of $4 \mathrm{~kW}$ [147].

The implementation of tiled aperture geometry for the CBC systems are mainly realized through four configurations: collimator arrays, micro-lens arrays, fiber bundle, and multicore fibers/photonic crystal fibers (MCFs and MC-PCFs). There are excellent demonstrations of CBC of multiple fiber lasers in an array scheme [148-154]. This architecture is a promising approach to power scaling by increasing the number of lasers. Figure 6 provides two main schematics illustrations of tiled aperture configuration, laser array and multicore fiber/crystal fiber.

- $\quad$ Multicore fibers and photonic crystal fibers (MCFs and MC-PCFs);

Combining $\mathrm{N}$ individual lasers in an array scheme needs $\mathrm{N}$ amplifier and $\mathrm{N}-1$ phase modulators, causing serious complexity in the operation. In contrast, MCFs and MC-PCFs have provided enormous potential for CBC such as high fill factor along with a common pump and phase modulation [155,156]. In this scheme, multiple cores are set in a single fiber/crystal fiber. Evanescent-coupling of the optical beam between cores leads to a coherent combination of the multiple beams [157]. Phase-locking in the MCFs/MC-PCFsbased systems can be obtained by active or passive methods such as the Talbot effect and 1-to-N-way phase-locking techniques [158-160]. In the passive operation, the beams are coupled to each other and the phase-locking happens by supermode selection [161,162].

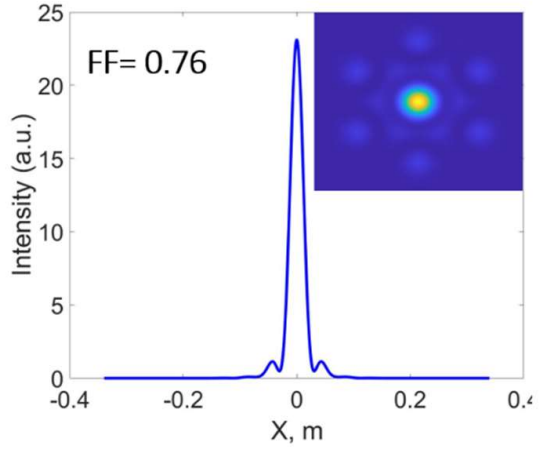

(a)

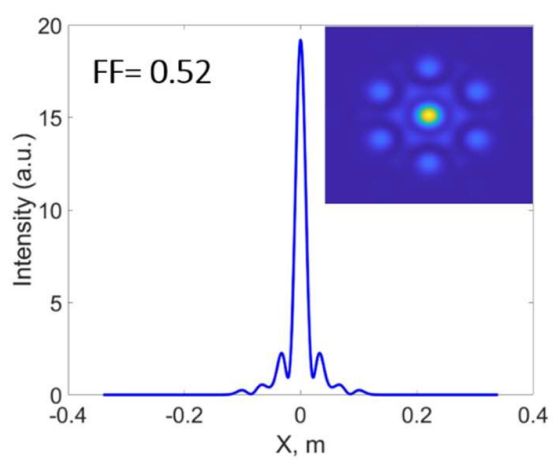

(b)

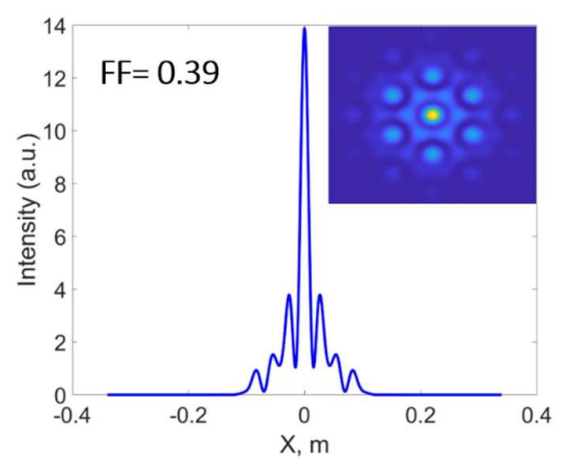

(c)

Figure 5. Impact of fill factor on the far-field intensity patterns of the coherent combination of seven beams in tiled-apperture geometry. The $2 \mathrm{D}$ beam profiles shown in the insets are illustrated in the same frame scale. 
However, in the passive mode, the power scaling is limited to the pump power coupling to the MCFs from a single diode. This problem was solved with active phase locking via digital holography [163]. In an investigation of power/energy scaling of coherently-combined multicore fiber amplifiers, the maximum achievable average power and pulse energy were calculated as $26 \mathrm{~kW}$ and $450 \mathrm{~mJ}$, respectively [164]. Moreover, MCF has great potential for the generation of structured light, for example, in 2020, Lin et al. in a proofof-principle experiment demonstrated the generation of structured light via MCF based on the CBC concept [165].

In conclusion of tiled aperture geometry, this configuration has been demonstrated as a promising approach for many applications such as high-power laser material processing and directed-energy systems. There has been a remarkable progressive development in CBC by implementing TA geometry. In 2016, Weyrauch et al. managed to combine 21 fiber lasers with adaptive mitigation of atmospheric turbulence effects over $7 \mathrm{~km}$ [166]. In 2020, by the coherent combination of 32 fiber laser at $1064 \mathrm{~nm}$, a $16 \mathrm{~kW}$ dynamic laser beam was obtained which opened new doors for advanced materials processing applications [167]. Recently, a $7.1 \mathrm{~kW}$ coherent combined beam was demonstrated via seven narrow-linewidth, linear-polarized fiber lasers with the filling factor of $95 \%$ and $18.8 \%$ energy proportion of the central lobe [168]. They reported that the peak intensity of the coherent combined beam was $\sim 22$ times higher than that of a single beam and $\sim 4$ times higher than that of an incoherent combined beam.

\subsubsection{Filled aperture (FA)}

This technique is based on the near-field beam combining of laser beams and is not affected by fill-factor limitation. This indicates that by implementing the filled-aperture CBC technique, nonuniformity in the near field intensity is eliminated and high combining efficiency (above $90 \%$ ) is promised. As a case in point, in 2020, Muller et al. reported a $10.4 \mathrm{~kW}$ coherently fiber laser system with $96 \%$ combining efficiency [169]. In general, laser beams can be combined in free space or in a guiding wave medium (such as a fiber coupler). However, owing to the strict power scaling limitation for guiding wave mediums, the free space CBC of fiber lasers is reviewed in this study. Implementation of the filled-aperture CBC scheme in free space can be classified in binary ports or multi-ports optical elements. Moreover, these combining techniques can be classified as polarization/intensity dependence combinations. In the filled aperture scheme, the most prominent optical elements for $\mathrm{CBC}$ are intensity beam combiners, polarization beam combiners, thin-film polarizers, diffractive optical elements, and segment mirrors (see Figure 7).

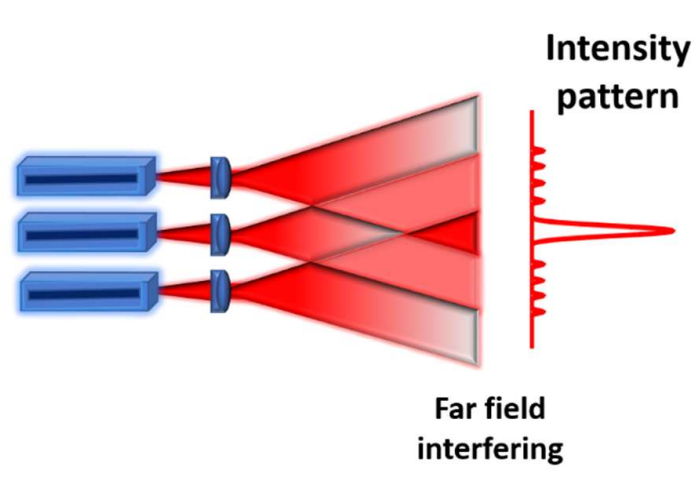

(a)

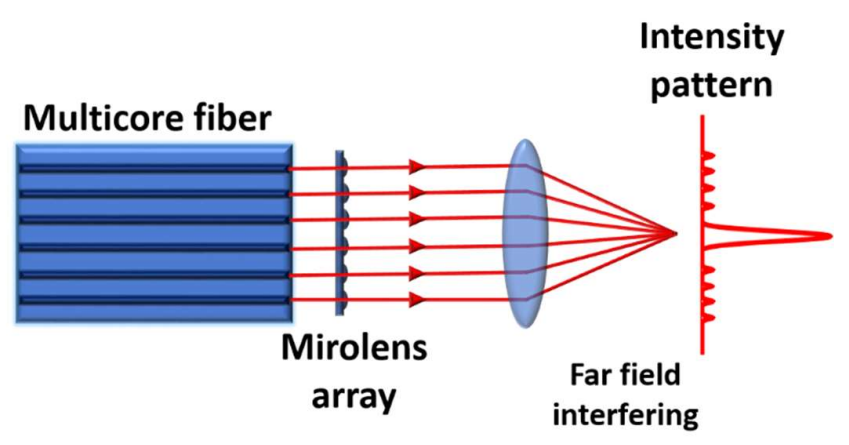

(b)

Figure 6. Schematic of tiled aperture coherent beam combination; a) CBC of multiple laser array in far field due to propagation and b) $\mathrm{CBC}$ of multiple laser beam emitted from multicore fiber in far field by implementing an optical lens. 
- Polarization beam combiners and thin-film polarizer

In polarization-dependent coherent beam combiners, two orthogonal linear polarized beams with fixed phase difference are combined in each step. The combined beam is yielded as a linear polarized beam which is rotated 45 degrees compared to the initial ones. With a rotation of 45 degrees of polarization direction by implementing a half-wave plate (HWP), the combined beam can be used in a cascading combination scheme. It is worth mentioning that in lack of phase control, the polarization of the combined beam is undetermined, limiting the channel combination number to two. A combination of polarized beams can be done via polarization beam combiners or thin-film polarizers. Several representatives on power scaling of both $\mathrm{CW}$ and pulsed laser via polarization beam combiners have been led to the realization of tens of millijoules energy and $\mathrm{kW}$ level average powers $[16,104,108,170-176]$. In 2010, Seise et al. in a proof-of-principle experiment demonstrated the combination of two femtosecond fiber lasers with the combining efficiency of $97 \%$ [171].

- Intensity beam combiners

The intensity beam splitters can be utilized as intensity beam combiners in the $\mathrm{CBC}$ scheme. IBSs are partially reflective and transmissive, for instance, in a 50:50 IBS, 50\% of each incident beam reflects and 50\% transmits. As a splitter, 50:50 IBS divides a beam into two entirely identical beams with the same properties. By controlling the phase of two entirely identical incident beams into a 50:50 IBS, theoretically, it is possible to achieve any arbitrary ratio from the output ports, from $0-100 \%$. It indicates the possibility of realizing an entirely destructive interference in one port (idler port), and consequently a fully constructive interference in another port (main port). To realize the maximum combining efficiency, the incident beams should have the same characteristics such as polarization and optical power. In combination of two completely identical laser beams except in output power, IBS with unequal ratios can be utilized. As a case in point, Muller et. al has reported a $10.4 \mathrm{~kW}$ coherently combined femtosecond fiber laser by utilizing 50:50 and 66:34

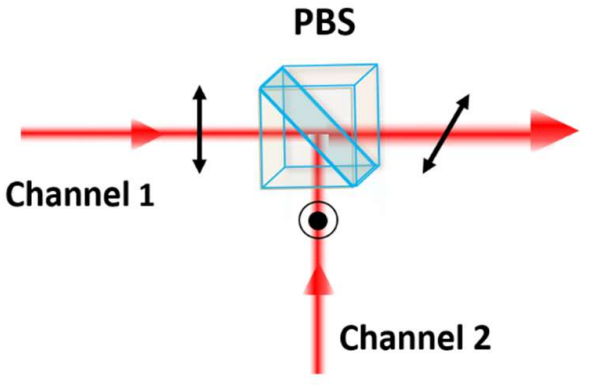

(a)

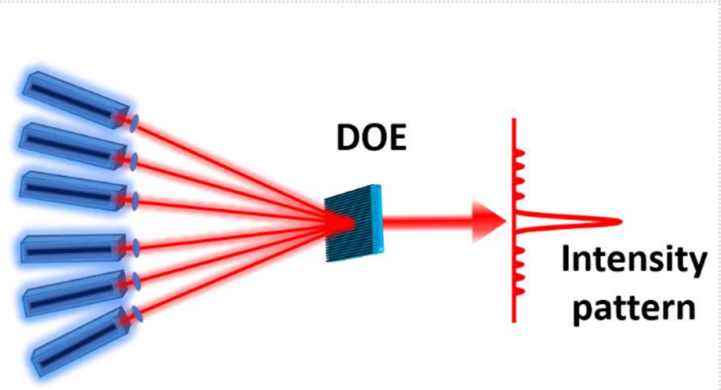

(c)

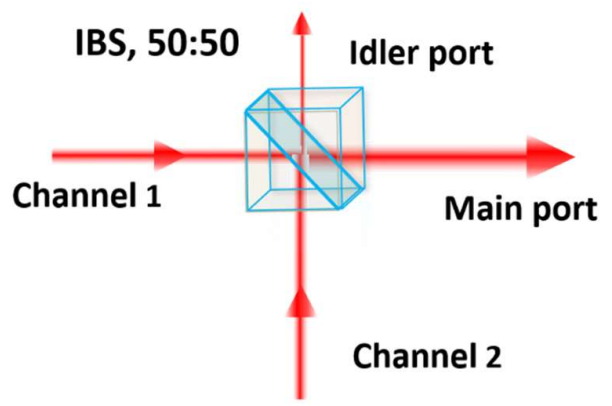

(b)

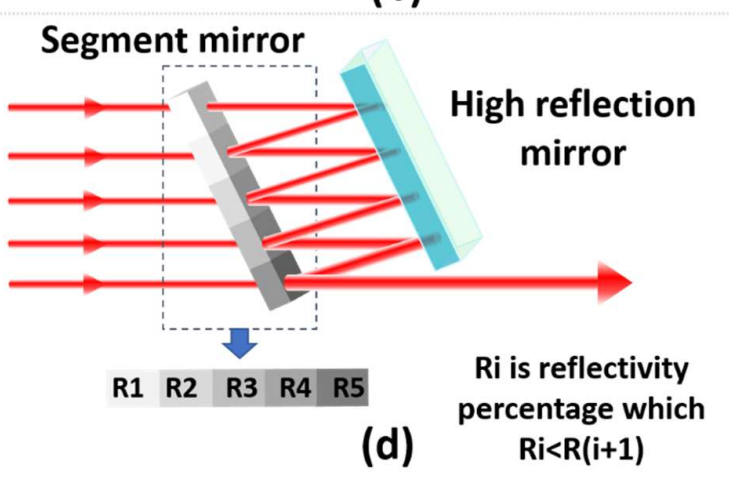

Figure 7. Schematic of filled aperture CBC systems by the four most popular optical elements; a) CBC with polarization beam splitter (PBS), b) CBC with intensity beam splitter (IBS), c) CBC with dif-fractive optical element (DOE), and $\mathrm{d}$ ) and $\mathrm{CBC}$ with segment mirror. 
IBSs in a tree-type combining/splitting configuration [169]. Compared to polarization beam combiners, IBSs have lower coating absorption, making them a viable option for high power $\mathrm{CBC}$.

In 2021, Muller et al. analysed the scaling potential of the beam splitters-based CBC, indicating the potential of realizing $100 \mathrm{~kW}$ average power via IBSs in binary-tree arrangement with maintained near-diffraction-limited beam quality (M2 $\leq 1.1$ ) and only $2 \%$ loss of combining efficiency [177]. However, this amount was reported as $33 \mathrm{~kW}$ for CBC via TFPs in binary-tree arrangement, since the effective absorption coefficient of TFPs is about 3 times larger than the IBSs. Moreover, a step forward in the case of IBSs, they realized that with the cost of 0.1 degradation in beam quality, a record of $300 \mathrm{~kW}$ average power at $90 \%$ combining efficiency with a beam radius of $1 \mathrm{~cm}$ will be achievable. In addition, with consideration of Sapphire and diamond as alternate substrate materials instead of fused silica, it was reported that no significant advantage is expected in changing the substrate materials.

- Diffractive optical elements

Diffractive optical elements (DOEs) are one of the most prominent optical elements which have extensive application in beam splitting, beam combining, beam shaping, and pattern projection. A splitting DOE, 1: $N$, can split an input laser beam into multiple output beams $(N)$ with precise angular spacing at angles that represent the diffractive orders of the DOE. In combining DOEs, $N$ : 1, multiple phase-locked incident beams (in specific angles corresponding to the diffractive orders $m$ ) can coherently combine into a single beam. The first demonstration of using DOEs for CBC of multiple lasers was reported in 1986 by Veldkamp et al. [178]. Combining multiple laser beams can be obtained in both one or two dimensions (1D and 2D). Theoretically, combining efficiency of $99.3 \%$ and 98.6\% can be achieved for CBC of multiple lasers using 1D and 2D DOEs, respectively $[179,180]$. By considering the thermal issues, the upper limit of achievable average power via DOE-based CBC systems is estimated to be at megawatt-level, with a combined beam diameter of $<10 \mathrm{~cm}$ [133, chap. 1.4]. As a case in point, a $5 \mathrm{~kW}$ laser was achieved using CBC of 5 fiber amplifier via 1D DOE $(1 \times 5)$, with the combining efficiency of $82 \%$ and the beam quality of $M^{2}<1.1$ [181].

However, realizing high combining efficiency for CBC of ultrafast pulsed laser is limited due to dissimilar pulse front tilt (PFT), which DOEs introduce for each input beam [182]. PFT, also called angular dispersion, leads the combined beam to have a spatial frequency chirp due to the diffraction, and consequently causes a loss in combining efficiency. In 2017, Zhou et. al demonstrated a technique to correct the PFT via a pair of DOEs, in which the combination of DOEs cancels the PFT and simultaneously minimizes the uncorrected dispersions [183]. By utilizing pair of DOEs, they experimentally combined four 120 fs pulses, without degradation in combining efficiency and pulse width. They theoretically showed that by implementing two DOEs, combining pulses with widths down to $30 \mathrm{fs}$ can be achieved without the significant combining loss, while $300 \mathrm{fs}$ is the onset of remarkable combining loss for combining with a single DOE. There have been significant advancements in CBC of high-power fiber lasers via DOEs in both pulsed and $\mathrm{CW}$ fiber lasers in recent years, leading to multi $\mathrm{kW}$ output power in the $\mathrm{CW}$ regime and CBC of eight femtosecond laser in 2D [181,183-190].

The combining efficiency of DOE-based CBC architectures is extremely sensitive to the beam alignment. In this regard, Liu et al. developed a theoretical model for investigating the losses of combining efficiency regarding beam deviation (induced by the DOEmount-tilt error, emitter-incident angular error, and DOE-groove-tilt error), beam size, and the DOE period [191]. They found that combining efficiency is the most sensitive to the beam deviation induced by the DOE-groove-tilt error.

- $\quad$ Segment mirrors (SM)

In 2018, Klenke et al. introduced a new concept of sequential phase locking for CBC systems [192]. In this intensity-based beam combination method, beams are coherently added one by one via a segmented mirror and can be scaled up to a large number of 
channels. Segmented mirrors have several zones with different reflectivity perventages ( $\mathrm{Ri}$, which $\mathrm{Ri}<\mathrm{R}(\mathrm{i}+1)$ ) and can be designed for operation in one or two dimensions. The combination of a segmented mirror parallel to a high reflective (HR) mirror promises a technique to CBC of several parallel laser beams in a compact system. This technique requires a detector after each combining step to collect data for the phase-locking system from the non-combining beam parts (rejection parts). It is also possible to collect the data from the combined beams in each step (leakage of the HR mirror). However, scaling up the number of lasers is limited due to the manufacturing complexity of unique thin-film coating sections for each laser beam. In 2020, Aleshire et al. by numerical analysis demonstrated a simplified design that enables SM to combine a higher number of channels compared to the standard SM [193]. They have realized that amplitude mismatch has a minor effect on combining efficiency, and just by two or three unique coatings it would be viable to combine several tens of lasers.

\subsubsection{Mixed aperture (MA)}

In 2020, Prossotowicz et.al presented a new CBC geometry concept based on a pair of micro-lens arrays (MLAs) as a central combining element [141]. MLAs are usually utilized as a high-quality beam homogenizer. CBC system with MLAs is based on the theory of imaging homogenizer [194-196]. This approach is analogous to the filled-aperture geometry but has two major differences. The first is that the fill factor does not constrain the MA scheme, and the second is that there is a combining element in the MA scheme, making it resemble the filled aperture geometry. The basic notion of this approach was taken from the efficient beam splitting concept introduced in 1991 by Streibl et. al, in which a lenslet array was applied as phase grating, providing diffraction orders with equal intensity [197]. Indeed, Prossotowicz et.al utilized MLAs-base splitters in reverse as combiners, and then by using a spatial light modulator (SLM) as a phase shifter, they managed to combine the laser arrays coherently. In a proof-of-principle experiment, in the first step, they demonstrated a highly efficient coherent beam splitting of an input beam into 25 beams $(1: 5 \times 5)$. Then, these were coherently combined into a single beam $(5 \times 5: 1)$ with a combining efficiency of $90 \%$. Both the splitting and combining procedures were realized with MLAs. Figure 8 provides a graphical representation of the concept of $C B C$ via mixed aperture geometry.

Recently, a new method for dynamic focus shaping based on highly efficient CBC within mixed-aperture geometry has been demonstrated [198,199]. In this technique, by changing the absolute phases of each input beam, the power weights of diffraction orders

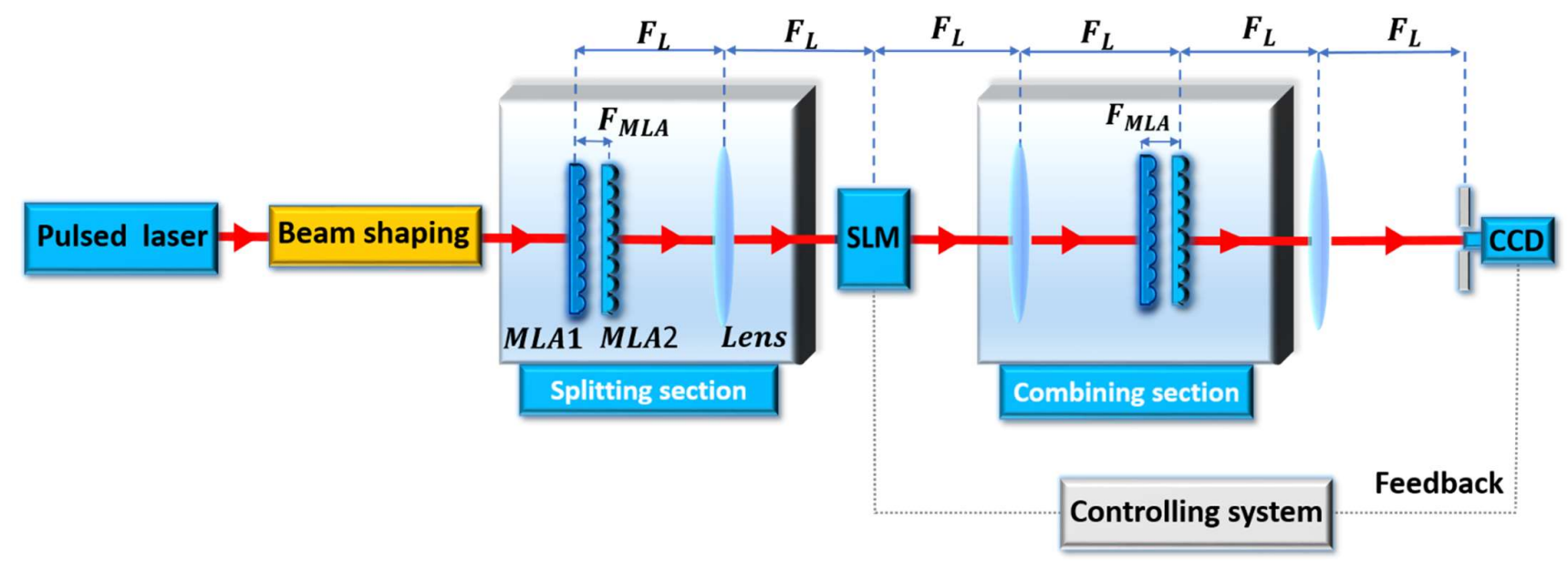

Figure 8. Schematic concept of mixed aperture CBC based on MLAs as a splitting and combining sections along with spatial light modulator as a phase shifter. 
are controlled. As a result, the number of combined beams along with their corresponding positions can be adjusted. In a proof-of-principle experiment supported by simulations, they conducted a highly dynamic beam switching as well as beam splitting and shaping in both $1 \mathrm{D}(1 \times 5)$ and $2 \mathrm{D}(5 \times 5)$. This approach has an enormous potential to scale up the power/energy performance with a speed of up to $10^{6} \mathrm{rad} / \mathrm{s}$.

\subsection{Laser sources and amplifiers}

\subsubsection{Seed lasers}

CBC of multiple lasers can only be achieved if all the laser beams are mutually coherent, which requires the same optical properties such as polarization state and spatial mode structure for all the laser beams. This mutual coherence can be realized within various techniques, in which splitting a single laser beam (seed laser), common resonators, and synchronising the multiple lasers via optical coupling are the most prominent ones. The latter can be achieved via multicore fibers. Splitting the laser signal can be realized by splitting optical elements such as IBS/PBS, or via all fiber couplers $(1 \times N)$. The common resonator technique will be discussed in detail in the section on passive phase locking. This section focuses on the seed lasers which are split in multiple identical laser beams via optical splitters.

To realize the highest combining efficiency, the OPD in each amplifier channel must be less than a fraction of the coherence length. Due to the inverse relationship between the coherence length and the laser linewidths, the narrow linewidth lasers provide a longer coherence length. However, it should be noted that (as discussed in the power scaling limitations due to the NLEs) SBS is the major limitation for power scaling of narrow-linewidth laser amplifiers. In the CW regime, the coherence length is usually longer than the induced OPD between channels. However, in pulsed lasers the coherence length is roughly equal to the pulse duration, highlighting the importance of optical delay path compensation for CBC of ultrafast lasers [103].

In $\mathrm{CBC}$ of $\mathrm{CW}$ lasers, narrow linewidth seed lasers are mainly fabricated based on fiber lasers or semiconductor lasers technology. Distributed-feedback (DFB) lasers have been demonstrated as a promising candidate for low phase noise, single frequency, singlepolarization seed laser for utilizing in high power MOPA systems [200,201]. In the case of pulsed operation, the seed lasers are typically mode-locked, gain-switched or semiconductor lasers.

\subsubsection{Laser amplifiers}

The amplification stage is one of the challenging parts of the $\mathrm{CBC}$ system due to high requirements to the target power level and threshold for nonlinearities. Based on the experimental reports, there are three main fiber-based solutions for amplifying the laser beams in CBC systems, including large mode area (LMA) fibers with a uniform longitudinal profile, photonic crystal fibers (PCFs), and taper double clade fibers (T-DCF).

- $\quad$ Large mode area (LMA) fibers

The development of LMA double-clad fibers rapidly boosts the output power/energy of fiber lasers in the last two decades, both in CW and pulsed modes $[1,3,7]$. It provides a higher pump injection to the fibers, which consequently has realized multi kW-level in average power and multi millijoules in energy within fiber laser and amplifiers. By analysing the thermal effect, nonlinear effects (including TMI), the upper limit for power scaling of fiber lasers have been predicted $28 \mathrm{~kW}$ and $52 \mathrm{~kW}$ for diode pumping and Tandem pumping technique, respectively [18]. The most common utilized active fibers in high power fiber laser systems have the $20-30 \mu \mathrm{m}$ and $250-600 \mu \mathrm{m}$ core and clad size, respectively. For example, LMA fibers are the gain medium for the world record of average power in ultrafast fiber laser systems, $10.4 \mathrm{~kW}$, in which 12 channels with ytterbiumdoped step-index fibers with $20 \mu \mathrm{m}$ core and $400 \mu \mathrm{m}$ cladding diameter were utilized in the main amplification stages [169]. 


\section{- $\quad$ Photonic crystal fibers (PCFs)}

Based on the above explanation on power scaling limitations, the amplification of laser signals has faced severe limitations due to NLEs, which are especially acute for amplification of ultrafast laser pulses with high peak powers. Therefore, an increase in the core size is a mitigation approach for the NLEs, providing more pump injection capability. However, increasing the core size has a major drawback due to maintaining single-mode operation and TMI. There has been much research on optimizing the fiber structure to keep the single-mode operation in the largest core size, mainly by decreasing the core NA of the fibers. Some technical challenges have limited the core NA to 0.06 in the commercial ones, although the ultra-low NA of 0.032 is reported by Midilli et al. in 2019 [80].

However, photonic crystal fiber (PCF) technology, which is also called air-silica microstructure or holey fibers, has opened new horizons in power/energy scaling in laser technology [202]. PCFs allows tailoring the NA via precise control of the index contrast, which resulted in a single-mode fiber within a large mode field diameter (MFD). In this case, MFD of a $35 \mu \mathrm{m}$, corresponding with mode field diameter (MFA) of $\sim 1000 \mu \mathrm{m}^{2}$ has been achieved for a low-nonlinearity single-transverse-mode in a PCF structure [203]. Moreover, PCFs has demonstrated as an ideal substrate for multi-core structures in CBC systems $[161,162,204]$. In an improvement in the PCFs technology, the large-pitch photonic-crystal fibers (LP-PCFs) concept was introduced for effective single-mode operation in PCF technology for power/energy scaling up [205]. Use of LP-PCFs have resulted in 22 GW peak power [16], $23 \mathrm{~mJ}$ single pulse [175], and $\mathrm{kW}$ level average power within CBC systems $[174,176]$. However, the major drawback of PCFs is high bending loss leading to the inconvenient large size of the system.

- $\quad$ Taper double-clad fibers (T-DCF)

In 2008, Filippov et al. introduced a new concept of double-clad fiber, called taper double clade fiber (T-DCF), which offered a new horizon to amplification of laser signals to high power/energy levels with maintaining the excellent beam quality [206-211]. In TDCFs, both core and clad layers vary in diameters along the fiber length. T-DCFs provide a varying large core diameter up to $200 \mu \mathrm{m}$, resulting in the minimum non-linear phase shift and the high pump absorption capability. Since the size of the core gradually increases along the fiber length, the mode content does not change through amplification, which resulted in excellent beam quality $\left(M^{2}<1.1\right)$. Indeed, T-DCFs have an inherent capacity to raise the NLEs thresholds [212]. However, for a stable high power/energy amplification, in addition to large MFD, polarization-maintaining is required. In 2018, Fedotov et al. presented the first ultra-large core $\mathrm{Yb}$-doped birefringent active T-DCF, with a core size of $96 \mu \mathrm{m}$, effective MFA of $2000 \mu \mathrm{m}^{2}$ and the beam quality of $M^{2}<1.09$ [213].

T-DCFs have been extensively advanced in recent years due to enomorous potentiality to deliver outstanding characteristics, resulting in multi $\mathrm{kW}$ in average power and millijoules level pulse energy. As a case in point, soon after the demonstration of T-DCF in 2008, a $600 \mathrm{~W}$ single-mode $\left(M^{2}<1.08\right)$ laser amplifier was reported by Filippov $e t$ al. in 2009 with an efficiency of $63 \%$ [214]. One year later, in 2010, they improved their work to a highly efficient $750 \mathrm{~W}$ laser amplifier with an efficiency of $80 \%$ [215]. Recently, a $4 \mathrm{~kW}$ CW single-mode laser via double-tapered double-clad fiber (DT-DCF) with the beam quality of $M^{2}<1.33$ has been reported [216]. Later, it has been increased to a $5 \mathrm{~kW}$ monolithic fiber amplifier, called spindle-shaped YDF, with an optical efficiency of $66.6 \%$ and beam quality of $M^{2}<1.9$ [217].

Regarding the production of single-frequency lasers, in 2020, a $550 \mathrm{~W}$ single-frequency fiber amplifier was demonstrated based on the polarization-maintaining T-DCF [218]. Moreover, $100 \mathrm{~ns}$ single-frequency laser pulses with $0.5 \mathrm{~mJ}$ energy and beam quality of $M^{2}=1.08$ were obtained [219]. In case of energy scaling, $1.6 \mathrm{~mJ}$ and $0.24 \mathrm{~mJ}$ energy pulses were achieved via actively and passively high-power Q-switched lasers, respectively $[220,221]$. The latter was the first demonstration of a Q-switched fiber ring laser 
which was based on the active polarization-maintaining tapered double-clad fibers (APMT-DCF).

There are several reports on utilizing T-DCFs to simultaneously achieve the high peak and average power pulses in picosecond ranges with excellent beam quality, which have resulted in a few hundred watts in average power and megawatts level in peak power via T-DCF [222]. For example, in 2020, a laser with the $1.26 \mathrm{MW}$ peak power and $200 \mathrm{~W}$ average output power was obtained via implementing the T-DCF, without the need for additional stretching techniques [223]. These representatives indicate that $\mathrm{T}$ DCFs have great potential for high power amplification of the laser beams with maintaining the beam quality, which made them an ideal gain medium for powerful CBC systems [224].

\subsection{Phase locking systems}

The key requirement for $\mathrm{CBC}$ is that the laser arrays must be mutually coherent in both space and time to realize the maximum combining efficiency. Hence, controlling the phase of each laser beam is strictly necessary for a highly efficient CBC system. The unwanted variations in the optical phase, called phase noises, lead to a decrease in the combining efficiency. These phase noises can be caused by thermal effects, external environmental disturbance and so on. Figure 9 illustrated the far-field intensity patterns of the coherent beam combination of seven laser beams when the phase-locked loop is open and closed. As can be seen (Fig. 8(a)), when the phase control system is on (phase of all beams are controlled), the energy will be mainly concentrated in the main lob. However, without phase control operation, the energy will be distributed in random patterns. Phase control techniques are classed into two distinct categories based on the physical mechanism employed: passive and active phase control.

\subsubsection{Passive phase control}

In the passive phase-locking approach, called self-phase locking, cophasing is obtained based on a coupling mechanism that can be realized by energy coupling (self-organization of the laser emissions) or nonlinear interactions effects [160,225-237]. This approach is simple to design and operate. However, several physical limitations degrade the

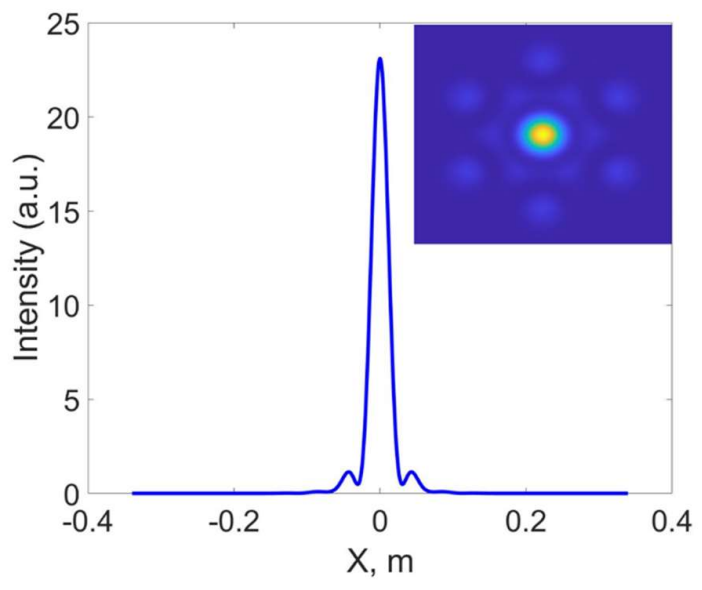

(a)

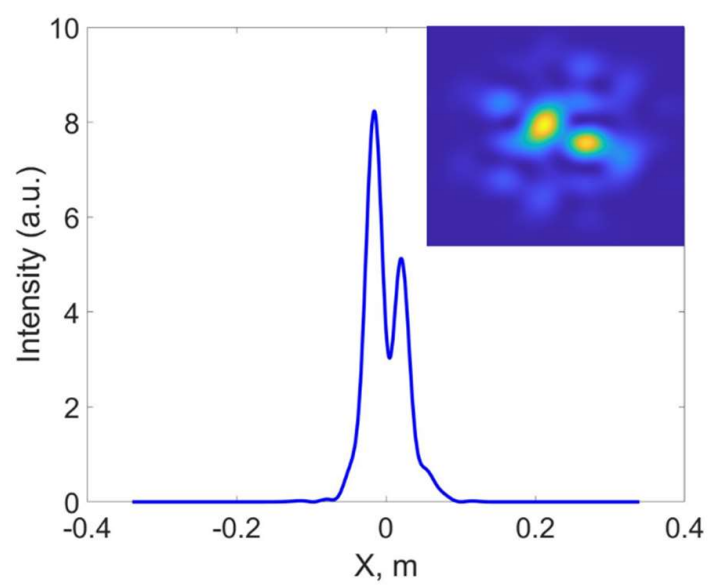

(b)

Figure 9. Far-field intensity patterns of coherent beam combination of seven laser beams, a) when the phase-locking system is on and b) when the phase-locking system is off. The 2D beam profiles shown in the insets are illustrated in the same frame scale. 
optical efficiency as the output power and the number of laser channels scale up [238,239]. Consequently, passive phase-locking methods are not suitable for implementation in high power/energy CBC systems. Hence, this paper mainly focuses on active phase-looking techniques and just reviews the most conventional passive methods in brief. To date, various research has been conducted on passive phase-locking CBC of fiber lasers [229,236,239-257]. The phase control in passive architecture can be classified into four distinct categories: a common resonator, optical phase conjugate (PC), evanescent (leaky) wave coupling, and self-organized technique.

- Common resonator

This technique works based on the supermode selection, which can be realized via spatial filters or the Talbot effect. The term "supermodes" refers to the phase-locked states, also called collective mode structures or array modes, and according to the coupled-mode theory (CMT), the array of $\mathrm{N}$ laser beams will have $\mathrm{N}$ supermodes. The in-phase supermode, where all the laser beams operating in the same phase, has the best beam quality and is the desired operation mode. In this method, all laser beams are amplified in individual gain mediums located inside a common optical resonator, and by provided feedback from the resonator, the beams are coupled to their neighbors through the supermode selection process. The main advantage of this method is that the optical power is distributed between several gain mediums. On the contrary, the major problem in this approach is the variation in the optical path, piston error, which hinders the power/energy scaling. Dammann grating cavity [258], self-Fourier cavity [233,259], Michelson cavity [260,261], and Talbot cavity [262] are the most well-known methods of common resonators passive phase-locking technique. In 2008, a $710 \mathrm{~W}$ passively phase-locked coherently combined laser system was achieved through a common ring oscillator, in which the common feedback was obtained from a spatially filtered sample [241].

- Optical phase conjugate

Optical phase conjugation, also known as time reversal, refers to a third-order nonlinear process that reverses the propagation direction and piston phase variation of an incident beam. By implementing phase conjugate mirrors (PCMs), distortion of the beam wavefront (piston phase) can be compensated, which are widely utilized in high power/energy laser systems. Optical phase conjugation is mainly realized by nonlinear optical effects such as degenerated four-wave mixing (DFWM) and SBS, of which the latter is the most commonly used approach. Since the first demonstration of coherent coupling of laser gain media using SBS-based PCM in 1986, stimulated Brillouin scattering phase conjugate mirrors (SBS-PCMs) have been greatly implemented in passive coherent beam combining systems [263-266].

- $\quad$ Evanescent (leaky) wave coupling

This technique is extensively utilized for CBC of semiconductor laser beams [267]. In this approach, the laser beams are positioned close to each other, so that their optical fields overlap and couple to each other through the evanescent wave coupling process. Consequently, the energy can transfer between the single gain mediums. Regarding the number of gain medium (N), N supermodes (collective mode structures) are allowed to excite in the system. Through the suppermode selection process, the in-phase supermode dominates the laser operation. The most prominent disadvantage of this technique is the limitation to scaling up the laser numbers. MCFs/MC-PCFs are the best representatives of the evanescent wave coupling technique [161,162,241,268,269]. In this regard, in 2000, Cheo presented the concept of clad pump multi-core phase-locked fiber laser for brightness scaling of the fiber lasers [270]. One year later, Cheo et al. reported a high brightness laser beam from a phase-locked multicore $\mathrm{Yb}$-doped fiber laser array under strong evanescent wave coupling with equal strength among all cores, which was the first in-phase supermode achieved via a 3D laser array [268,269].

- $\quad$ Self-organized 
A self-organized technique refers to a passive coherent combining technique that does not require active length, polarization, or amplitude control. The physical mechanism of this technique is based on the longitudinal mode selection in a Michelson interferometer resonator, which is applied for more than two channels. Indeed, this technique slightly deviates from the definition of the $\mathrm{CBC}$ concept, in which all the laser parameters must be identical. In fiber laser systems, this method was first applied for a coherent combination of multiple neodymium-doped fiber lasers in 2004, in which self-cophasing was achieved spontaneously via all-fiber $2 \times 2$ coupler trees [225,271]. In 2005, a 200 W CBC fiber laser system was demonstrated through the self-organized phase-locking technique using nonfiber couplers [240]. In 2007, Zhou et al. provided a model for the phase-locking evolution of each laser in a fiber laser array, which was analyzed theoretically and numerically [272]. One year later, Cao et al. researched the self-organized coherent fiber laser arrays with circulating field theory, in which the influences of the OPD and the number of fiber lasers on the performance of the whole system were discussed in detail [238]. In 2009, Chen et al. demonstrated a new self-organized coherent combining configuration of an array of fiber lasers, which was based on mutual injection locking [273,274]. However, the most prominent drawback of this technique is that the combining efficiency is reducing with the increase of channel numbers.

\subsubsection{Active phase control}

Active phase-locking systems consist of two sections, phase detection and active phase noise compensation. Phase corrections are mainly implemented by electro-optic modulators, liquid crystal modulators, and piezo-electric actuators. The main techniques for actively phase-locking systems can be classified into five categories, including HanschCouillaud (HC) polarization detection, optical heterodyne detection (OHD), frequency dithering, hill climbing, and Phase-intensity mapping (PIM).

- Hansch-Couillaud (HC) polarization detection

For polarization beam combination, HC polarization detection is the most widely implemented technique, in which the phase difference between two orthogonal beams is measured [275]. In this technique, a small fraction of the combined laser beam is directed to the setup called HC detectors, which consist of one PBS, one quarter-wave plate (QWP), and two photodiodes, which is shown schematically in Figure 10 . There is $45^{\circ}$ difference between the axis of QWP and the axis of PBS, leading to split up into two laser beams with orthogonal polarization. Two photodiodes located in each output port of the PBS measure the optical beam powers and create the electrical signals, proceeding to calculate their difference, called the detection signal. If the phase between the two optical beams is zero,

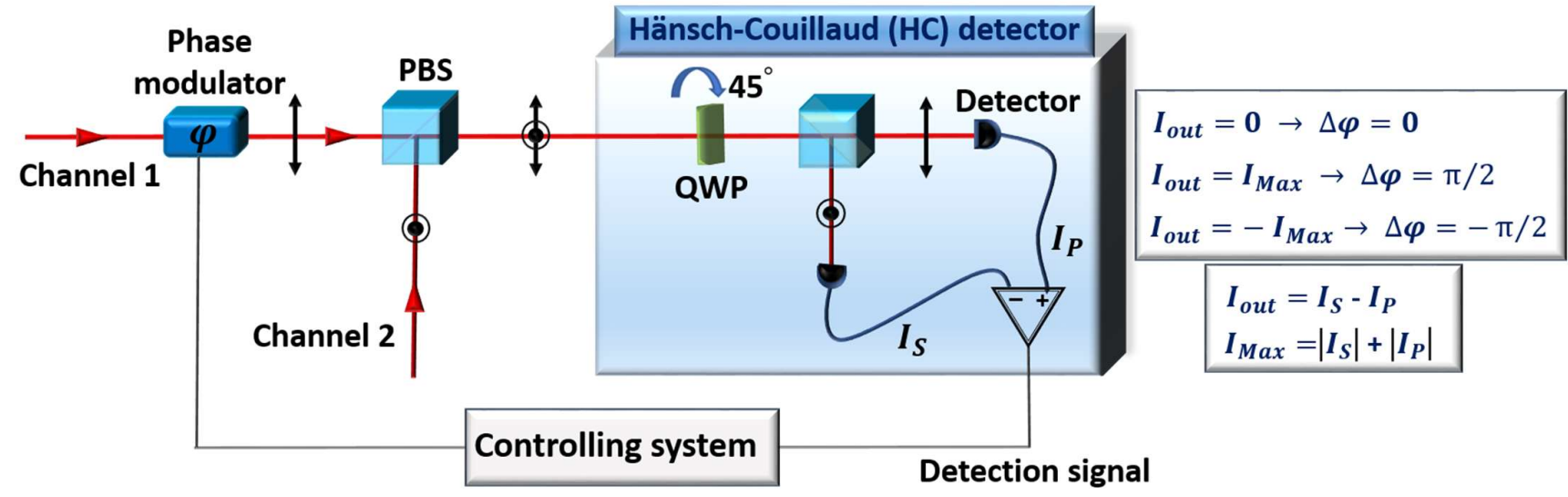

Figure 10. Schematic of Hansch-Couillaud (HC) polarization detection for PCBC of two channels. 
the detection signal would be zero. So, by this approach, the phase difference corresponds to the detection signal.

In an ideal PCBC of two orthogonal polarized beams with the same power and phase, the combined beam is completely linear polarized, rotated by $45^{\circ}$ compare to the initial input beams. If a sample of the combined beam is directed towards the HC-detectors, the QWP which is rotated by $45^{\circ}$ relative to the PBS axis creates a circular polarization beam. The PBS divided it into two orthogonal polarized beams with the same optical power. Then, the detection signal will be zero at this state, indicating the perfect co-phasing between the channels. As a drawback of this technique, one HC-detector for each two-channel combination step is required. This technique has been mainly applied for CBC of pulsed lasers, and through this phase-locking technique, the records of few tens of $\mathrm{mJ}$ energy, and $\mathrm{kW}$ level average power in CBC of ultrafast fiber lasers have been obtained $[175,176]$.

- Hill Climbing

Hill climbing is a mathematical optimization algorithm, intending to optimize the target parameter, which is the laser output power in CBC systems. This technique is the simplest method for active phase-locking of multiple laser beams, in which the cophasing is achieved by maximizing the combined laser power in the far-field central lobe. Indeed, no phase detection is needed in the hill-climbing optimization algorithm, and the phase of each laser amplifier is controlled concerning other laser amplifiers. The stochastic parallel gradient descent (SPGD) optimization algorithm is the most utilized in this technique [235]. In the SPGD algorithm, by applying the initial random set of slight shifts to phase modulators, along with getting feedback from the combined beam, the effect of the phase changing on combined power is detected. Based on this feedback, the new set of slight shifts is applied to maximizing the combined power. This procedure continues until the power (efficiency) is maximized. SPGD technique requires a single detector to get the feedback, leading to simplicity in operation. For many years, the slow convergence rate of the SPGD algorithm was considered as its major drawback. However, with great advancement in optimization of this algorithm, this technique has been achieved significant progress to scale up the number of laser channels. Up to now, the maximum number of coherently combined laser beams of 107 channels has been achieved by the SPGD algorithm [154]. Moreover, recently $7.1 \mathrm{~kW} \mathrm{CBC} \mathrm{CW} \mathrm{laser} \mathrm{system} \mathrm{has} \mathrm{been} \mathrm{demonstrated} \mathrm{by} \mathrm{imple-}$ menting the SPGD algorithm [168].

- $\quad$ Optical Heterodyne Detection (OHD)

OHD method refers to an active phase-locking technique that involves a frequencyshifted reference beam, typically shifted by an acousto-optic frequency shifter or similar, along with a sample of laser beam array to produce an electrical signal (heterodyne signal) through an interferometric combination. This heterodyne signal provides the correlation phase information between the reference beam and the individual lasers. So, by implementing one phase modulator in each amplifier channel, the phase of the channels will control.

This method, also called coherent detection, requires one detector per laser channel. Despite the Hill climbing method, in OHD the phase-locking process of each laser channel is independent of other channels, indicating all individual lasers are locked directly to a reference beam. Therefore, no physical limitation is imposed upon the scaling of the number of channels. OHD is applied in both tilled and filled aperture geometry, although utilizing it in the context of the tiled aperture is more convenient. Due to feasibility in the application, the OHD technique was utilized in various CBC systems [116,184,276]. As an example, OHD was implemented as an active phase-locking technique for the first 100kW CBC laser system [138]. However, the requirement for the detector array arrangement along with the necessity of the reference beam constrains the performance of the OHD technique. Although, the most prominent drawback of the OHD technique is the lack of a direct way to confirm all the channels are phase-locked to each other. 
- Frequency dithering

Frequency dithering refers to an algorithm in which sinusoidal phase modulations with unique frequency/frequencies are implemented to derive the phase errors by radio frequency $(\mathrm{RF})$ demodulation. This technique can be implemented through a single or multi-frequency dithering context.

In the multiple dithering technique, called synchronised multidither, tagging a small phase dither to all the laser beams along with the sampling from the combined beam via a single detector provides a superimposition of beats due to interference between the laser beams. By implementing the radio RF demodulation, an optical phase relation between each pair of lasers will be obtained. Consequently, after analyzing these phase relations, phase-locking will be achieved through adjusting the optical phase via implementing one phase modulator in each amplifier channel. Unique frequencies are provided by controlling the voltage of electro-optic modulators (EOM). This technique was first utilized in adaptive optics, called coherent optical adaptive techniques (COAT), for mitigating the phase distortion induced by atmospheric turbulence [277]. This technique is completely independent of the need for a reference beam. Requiring only one detector for sensing the phase shift between the laser array leads to scalability in implementing this technique for multiple channels. Furthermore, synchronous multidither approaches are free of phase set point ambiguity, which is intrinsic to the OHD method. The most prominent disadvantages of this method are the electronic complexity and cost.

This technique has been significantly developed to improve accuracy and reduce complexity in the last decades. In this regard, in 2010, the concept of the single-frequency dithering technique was introduced, in which one modulation frequency and consequently one phase control module are required [278]. This technique boosts the function of CBC for larger number of channels $[279,280]$. Furthermore, in 2014, an active phaselocking using a square wave dithering algorithm was demonstrated, which no longer need the RF demodulation circuit and is eminently suitable for large scale CBC systems [281].

Among synchronous multidither phase-locking techniques, locking of optical coherence via single-detector electronic-frequency tagging (LOCSET) is a commonly used algorithm. LOCSET technique can be operated in the context of self-referenced or self-synchronous [282,283]. Various research has been conducted on CBC laser systems based on the LOCSET technique $[169,175,181,186,187,192,278,284-286]$.

- Collective phase-intensity technique

1. Collective phase measurement technique

For scaling the number of laser channels, direct measurement of the phase information is needed. This imposes complexity on the systems, which implement single-detector-based phase-locking techniques. In this regard, the idea of implementing a camera as an array of detectors for measuring the intensity of interferometric pattern resulted in a new phase-locking technique with great potential for boosting the control speed and scaling the channels number. The phase difference between channels can be calculated from the comparison of the ideal interferometric pattern with the interferometric pattern formed by amplified beams. In 2011, the record of the 64-channel CBC system was reported by Bourderionnet et al., in which the phase of each fiber was individually controlled [148]. The phase information for each channel was obtained by implementing a quadriwave lateral shearing interferometer (QWLSI) as a self-referenced wavefront analyzer.

2. Phase-intensity mapping (PIM)

In 2015, Kabeya et al. presented a new method for active phase-locking, in which the phase differences are converted to the intensity pattern through an optimization algorithm [287]. In this method, there is no phase measurement and consequently, it does not require adding phase modulations or phase perturbations on the phase pattern during the iteration steps. Therefore, this method cannot be considered among the four previous phase-locking techniques. In a proof of principle experiment, they managed to actively 
control the phase of the $4 \times 4$ laser beam array. Then, in an improvement, they demonstrated a CBC of 37 fiber amplifiers with the combining efficiency of $94 \%$, by implementing the PIM technique in an optimization loop [151].

In 2019, Du et al. demonstrated a new version of the PIM-based phase-locking algorithm based on intensity pattern recognition of the uncombined beams [190]. One year later, they managed to produce 81 independently controllable beams and then coherently combined them via DOE [153]. This technique has had great potential for boosting feedback control by implementing machine learning, which recently has resulted in the stabilization of the 81- channel CBC system with the control speed of tens of times faster than the SPGD algorithm [288]. Moreover, they have developed a new approach for boosting the feedback phase control of high channel-count CBC by implementing a programmable array generator [289].

\subsection{Optical path difference control}

The OPD between the lasers in the CBC scheme has a detrimental impact on the combining efficiency. The OPD induces a phase shift and reduces the temporal overlap of the optical pulses, which in turn leads to a decrease in the combining efficiency of the CBC system. The effect of OPD in pulsed lasers is more perilous rather than in CW lasers, which may lead to the entirely losing temporal overlap of the pulses [103,290,291]. In this regard, the shorter pulses (broader bandwidths) are more sensitive to OPD, resulting in more loss in combining efficiency. For instance, to realize a combining efficiency of $90 \%$ for the pulse with a bandwidth of $5 \mathrm{~nm}$ (corresponding to the transform-limited pulse duration $\approx 310 \mathrm{fs}$ at $1030 \mathrm{~nm}$ ), the OPD must be less than $25 \mu \mathrm{m}$. In comparison for a pulse with double the bandwidth at $10 \mathrm{~nm}$, this OPD difference requirement decreases to $12 \mu \mathrm{m}$. Furthermore, path length differences in CBC of pulsed lasers can induce extra phase difference between different pulses owing to the SPM and group velocity dispersion (GVD consequently decreasing the combining efficiency[103,290,291]. Hence, compensating the OPD in the laser channel is crucial in the CBC systems.

The primary technique for compensating the OPD is to add a delay line by splicing the extra fiber to the short channels, which might be highly suitable for the CW and nanosecond laser pulses with GHz linewidth [290]. However, for fine adjustment which is needed for the CBC of ultrafast lasers, the variable optical delay line (VODL) with a resolution of less than $1 \mu \mathrm{m}$ is desired [146,152,172,292]. Due to the limited range of the VODLs, first, the OPD must be adjusted manually (for example by splicing the extra passive fiber), so that VODLs realize the fine adjustment only. There are commercially available electronically controlled delay lines (ODL) capable of compensating for up to $4000 \mathrm{ps}$ delay range, with the resolution of $<1 \mathrm{fs}$. These motorized ODLs consist of an input and output fiber collimator and a movable reflector. The input light propagates in free space and is reflected from the movable mirror and coupled to the output fiber collimator. The OPD compensates in free space through separation between the input and output ports by moving the reflector (see Figure 11).

It is worth noting that from a fundamental perspective, the OPD and phase control act on the same quantity: time delay between the combined electric fields. Indeed, the OPD control system performs the coarse and fine adjustment of optical path differences between the laser beams. However, OPD adjustment on the wavelength scale is performed by the phase control system. In some systems, optical phases are controlled by electro-optical phase modulators [284], which create ultrashort OPDs by changing the refractive index of the propagating medium. In some other cases, ultrashort OPDs (phase control) are controlled by implementing the piezo-actuated mirror (PA) [169].

\subsection{Channel scaling}

To boost the power/energy of the CBC systems, there are two main strategies: scaling the power/energy of the individual laser channels and increasing the number of laser channels. The first one has been hindered by several physical limitations, such as NLEs, 


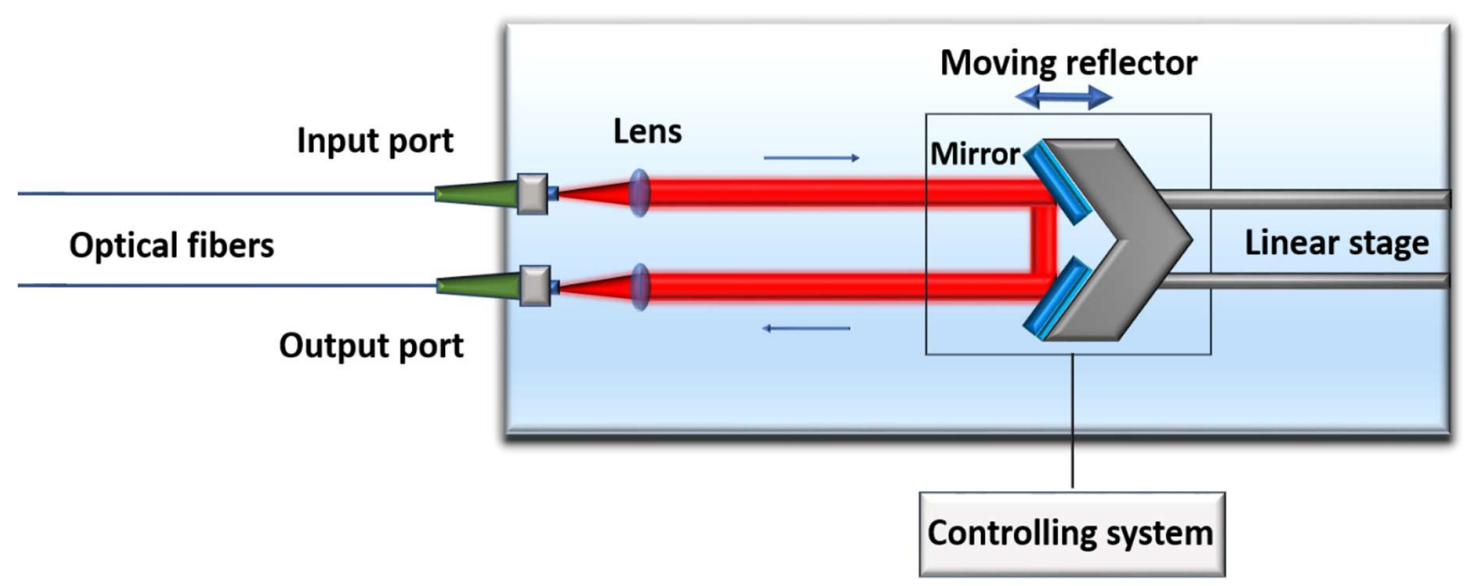

Figure 11. Schematic of compensating OPD via a variable optical delay line (VODL).

optical damage, and thermal issues. Therefore, increasing the number of lasers has consistently been in demand for scientists. However, increasing the laser numbers has faced several limitations, owing to the signal error in the system for the phase-locking algorithm. Indeed, by increasing the number of channels, the combining efficiency will decrease due to the increase of system errors and simultaneously decreases the bandwidth of the phase-locking algorithm.

There has been an ongoing effort to increase the number of combining channels, summarized in table 1. In 2006, Yu et al., combined 48 CW Polarization-maintaining (PM) fiber lasers [293], and the number was increased to 64 lasers in 2011 with a residual phase error of $<\lambda / 10$ RMS [148]. This improvement has also been demonstrated for the pulsed lasers, resulting in a combination of 61 femtosecond lasers with the residual phase error of $\lambda / 90$ RMS. Then, the number of channels reached 81 within RMS error of $<0.1 \%$ [152]. However, the first demonstration of CBC of more than 100 lasers was achieved by combining $107 \mathrm{CW}$ fiber lasers with the phase residue error of $\sim N 22$ RMS and $22.5 \%$ ratio of power in the central lobe.

Table 1. Representative results of the channel scaling in the $\mathrm{CBC}$ of fiber lasers.

\begin{tabular}{|c|c|c|c|c|c|c|c|}
\hline Year & Channel & geometry & $\begin{array}{l}\text { Operation } \\
\text { mode }\end{array}$ & $\begin{array}{l}\text { Combining } \\
\text { efficiency }\end{array}$ & $\begin{array}{l}\text { Controlling } \\
\text { system }\end{array}$ & Institution & Ref. \\
\hline 2006 & 48 & Tiled & $\mathrm{CW}$ & $\begin{array}{c}\text { RMS erorr }< \\
\lambda / 30\end{array}$ & SPGD & MIT & [293] \\
\hline 2011 & 64 & Tiled & $\mathrm{CW}$ & $\begin{array}{c}\text { RMS erorr }< \\
\quad \lambda / 10\end{array}$ & PIM & TRT & [148] \\
\hline 2017 & 37 & Tiled & $\mathrm{CW}$ & $96 \%$ & PIM & UNILIM & [151] \\
\hline 2020 & 61 & Tiled & Pulsed & $\begin{array}{c}50 \%, \text { RMS erorr } \\
<\lambda / 10\end{array}$ & SPGD & IPP & [152] \\
\hline 2020 & 81 & Filled & Pulsed & RMS error $<1 \%$ & PIM & LBNL & [153] \\
\hline 2020 & 107 & Tiled & $\mathrm{CW}$ & $96 \%$ * & SPGD & NUDT & [154] \\
\hline
\end{tabular}

\footnotetext{
* The fringe contrast of the far-field intensity pattern.
} 


\section{Coherent beam combining of ultrafast fiber lasers}

As discussed earlier, amplification of ultrafast fiber lasers faces severe limitations such as NLE and optical damage, which hinders scaling up the peak and average power of the ultrafast pulses. CBC concept provides the possibility of distributing the laser power in multiple channels in space and multiple pulses in the time domain. However, due to the fast phase variation inside the pulses, $\mathrm{CBC}$ of ultrafast pulses are more challenging than the $\mathrm{CBC}$ of $\mathrm{CW}$ lasers. This is due to fact that not only the phase of the pulses must be locked to each other, but also the time delay between the pulses must be compensated to obtain the maximum combining efficiency. By utilizing VODLs, perfect pulse synchronization can be obtained. Another issue is that pulse operation induces intensity fluctuations in the feedback signals, which must be eliminated by the phase control system to prevent mixing with actual phase signal errors among each laser channel in the phaselocking process. For this purpose, low pass filters are implemented, eliminating the highfrequency intensity feedback induced by the high repetition rate pulsed lasers in the feedback loop.

The first reports of implementing $\mathrm{CBC}$ in pulsed lasers were in nanosecond pulses demonstrated by Cheung et al. in 2008 via utilizing a CW laser as a reference beam [137]. Then, in 2011 [294], Lombard et al. reported a pulsed laser system, in which 100 ns pulses were amplified in two separate channels and then coherently combined. However, the first demonstrations for implementing CBC in ultrafast pulses were reported by Seiz et al. in 2010 [171] and by Daniault et al. in 2011 [284], for the CBC of two femtosecond laser pulses. Chirped pulse amplification (CPA) is the most prominent strategy to realize the high power/energy pulses through the $\mathrm{CBC}$ systems.

The CPA technique was first demonstrated by Strickland and Mourou in 1985 [14]. It is based on a pulse stretching in time, which decreases its peak power and enables an amplification to the highest achievable level (see Figure 12). After the amplification stage, the stretched pulse is compressed down in time to its initial duration. There are several techniques for stretching and compressing pulses, classifying based on the utilized optical

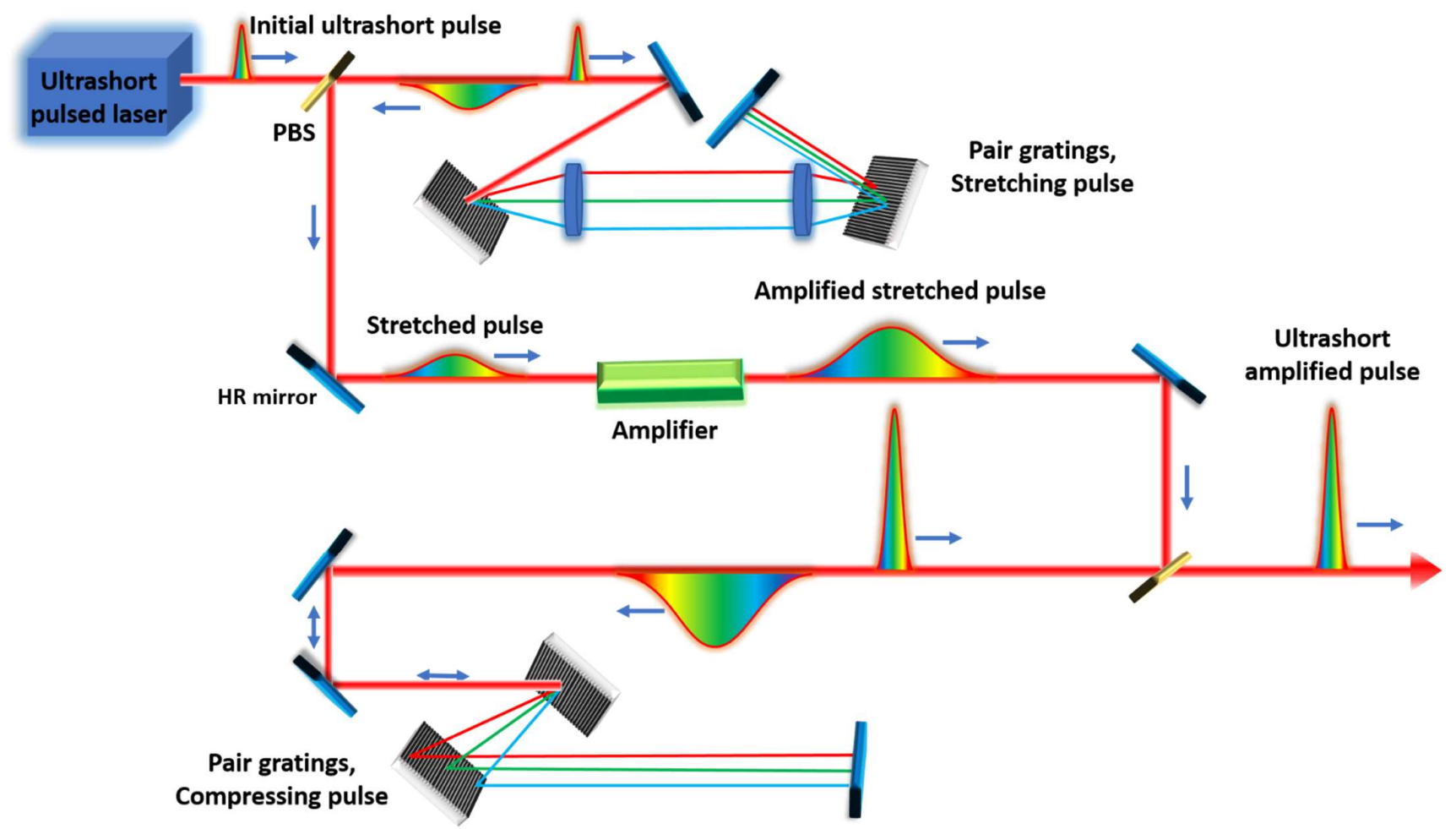

Figure 12. Schemes of chirped pulse amplification concept based on the pairs of gratings. 
element such as prisms, gratings, and optical fibers. Among them, bulk gratings have been established as a great option, due to the capability of strong stretching and compression. This technique has boosted the amplifying of the ultrafast pulses and has opened new horizons in laser technology, leading to the Nobel prize in physics in the year 2018. The CPA technique allows the achievement of high-power levels, and it is widely utilized in laser systems based on the CBC approach [175,176].

CBC of ultrafast fiber lasers can fall into one of the following architectures, including spatial, temporal, multidimensional, and spectral synthesis, based on the physics of the laser combination.

\subsection{Spatial $C B C$}

Spatial CBC refers to splitting the amplification to physically (and spatially) separate amplifier channels that are then combined. In this configuration, which represents the fundamental feature of the CBC systems, the initial laser pulse split (in space) into multiple replicas (N pulses) via splitting elements such as IBS, PBS, and fiber couplers. After amplification in the $\mathrm{N}$ parallel amplifier, pulses are combined to realize a high peak/average power single pulse (Figure 13). Several great demonstrations of the CBC of ultrafast

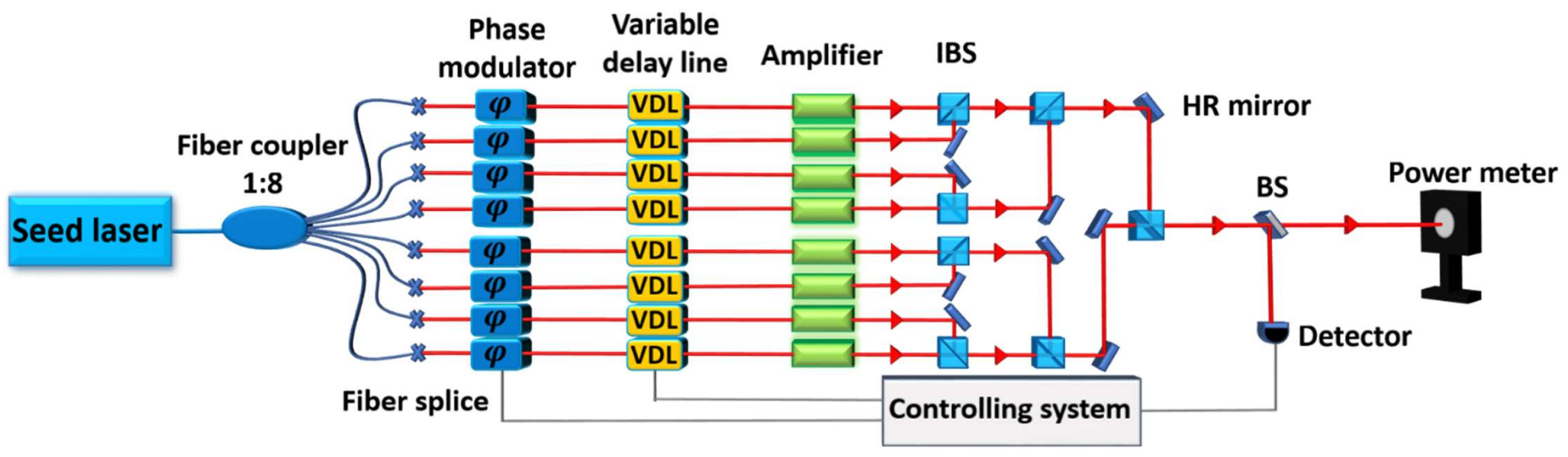

(a)

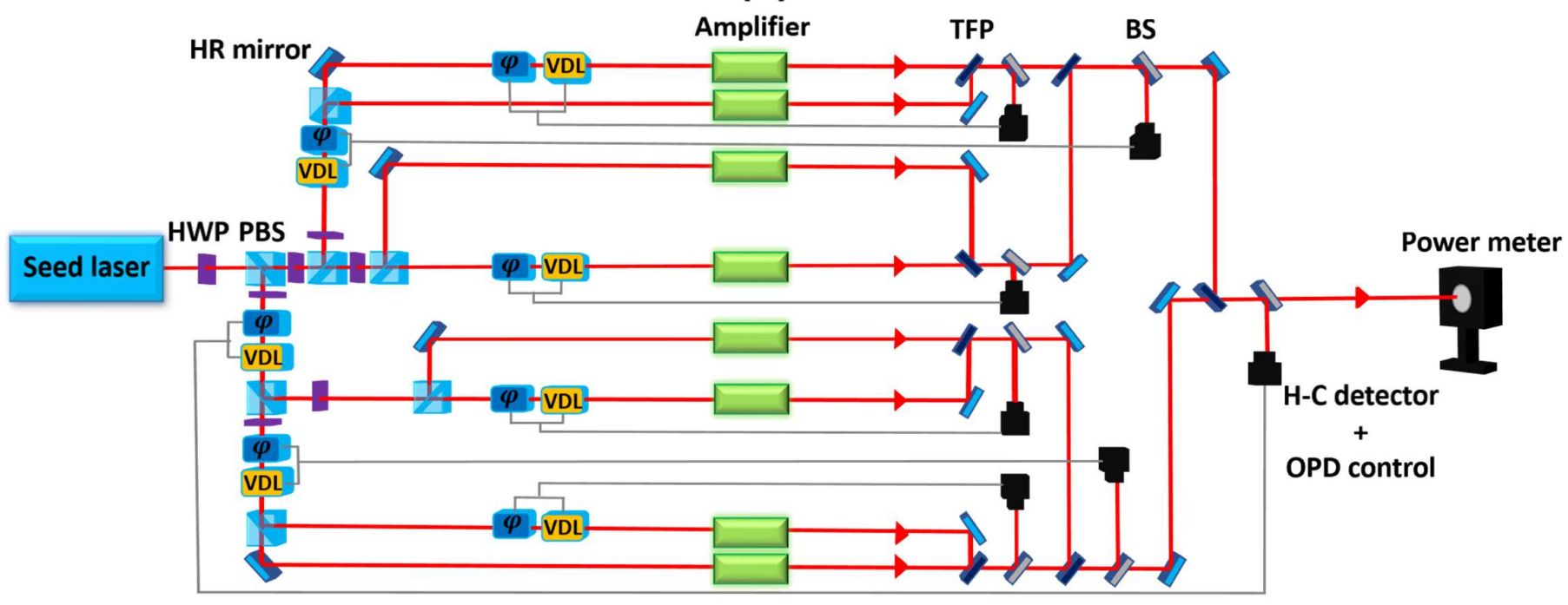

(b)

Figure 13. Schematic representation of spatial coherent beam combining; a) PCBC with PBSs as splitters, TFPs as combiners and phase-locking system by implementing Hansch-Couillaud detectors, b) CBC using fiber coupler as a splitter, IBSs as combining elements and phase-locking systems by utilizing a single detector. 
fiber lasers with an unprecedented record of high power/energy pulses have been reported since the first representative in 2010, via both active and passive phase-locking mechanisms [16,156,171,172,245,284,295,296]. For instance, in 2014, a coherently combined 22 GW peak-power fiber laser based on the CPA system was demonstrated [16]. The highest average output power of a coherently beam combined ultrafast fiber laser system, 10.4 $\mathrm{kW}$, with $254 \mathrm{fs}$ pulses at an $80 \mathrm{MHz}$ repetition rate (0.5 GW peak power) was demonstrated in 2020 through the spatial CBC configuration [169]. In addition, recently $1 \mathrm{~kW}, 10$ $\mathrm{mJ}, 120 \mathrm{fs}$ ultrafast fiber laser system has been reported through the spatial CBC of fiber CPA-systems [176].

\subsection{Temporal $C B C$}

In contrast to the $\mathrm{CW}$ regime where the power can only be distributed in space (i.e., splitting it to multiple laser amplifiers), in the pulsed regime, the replica pulses can be distributed both in space and time. Temporal CBC refers to splitting the pulse into several replicas in the time domain and amplifying these individual pulses in a single amplifier before combining, which is called divided pulse amplification (DPA), see Figure 14. Theoretically, by implementing the DPA technique, the peak power and the pulse energy can be scaled up by the factor of $\mathrm{N}$. This approach is a promising method for the energy scaling of ultrafast pulsed lasers, which was first demonstrated to mitigate the NLEs in amplification of ultrafast pulses by Zhou et al. in 2007 [297]. As can be seen in Figure 14, the initial pulse is divided into multiple replicas of the pulse with lower peak power and different polarization state compared to the initial ones. The purpose of pulse splitting is to reduce the nonlinear effects. Then, each pulse is amplified separately and finally combined coherently together. There has been various theoretical/experimental research on implementing the DPA technique, demonstrating numerous high power/energy ultrafast fiber lasers based on the DPA concept [173,175,298-302].

DPA technique can be realized in both active and passive mode. In a passive mode, the splitting and combining stages are implemented in the medium, relying on the double

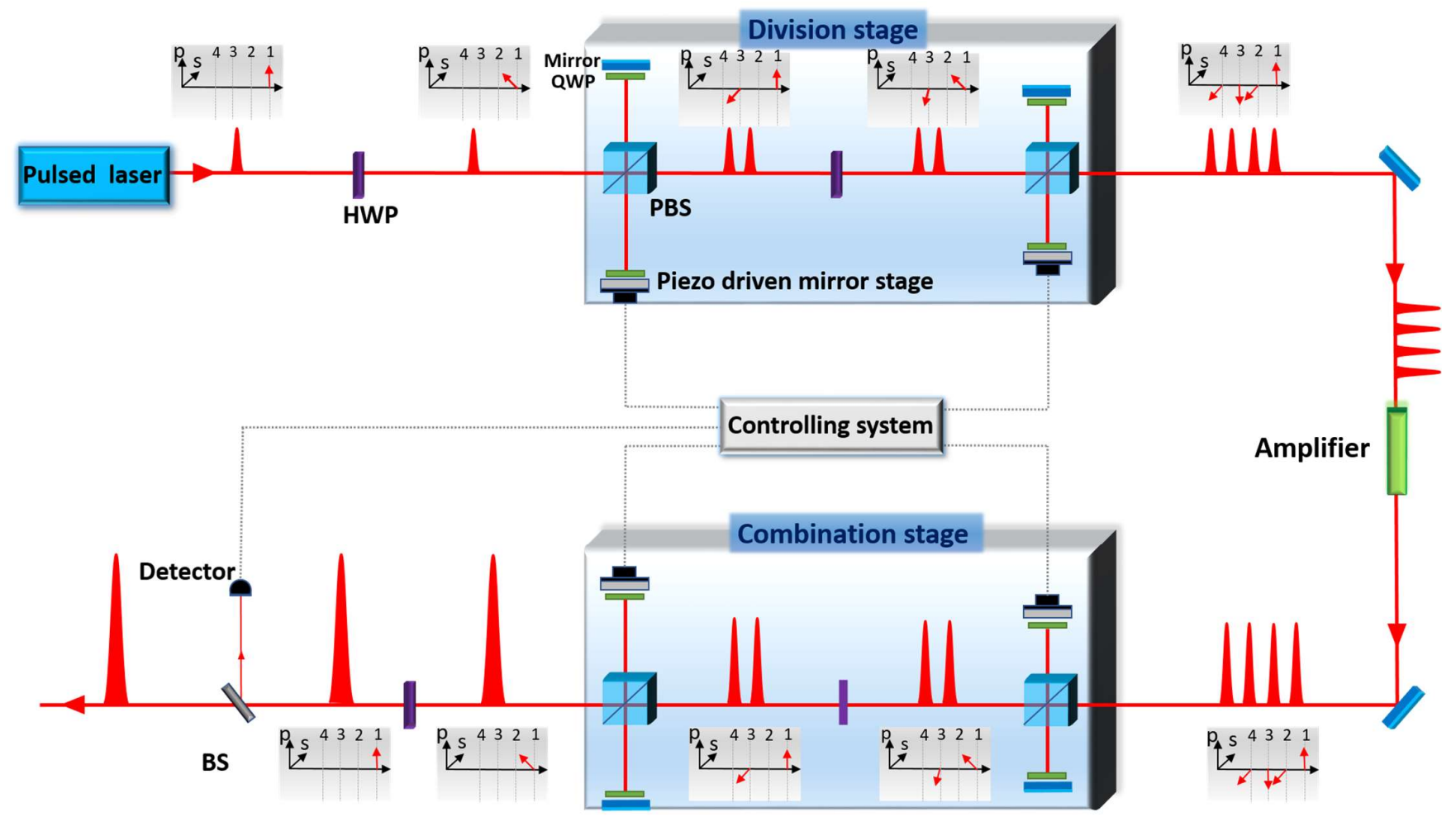

Figure 14. Schematic representation of divided pulse amplification (DPA) technique. 
passed configuration [300]. However, in a double-passed gain medium, the achievable pulse energy is limited to the gain saturation due to the asymmetric amplification. In 2014, Kienel et al. introduced the first experimentally demonstration of an actively controlled DPA system in which the splitting and combining stages were separate [301].

\subsection{Multidimensional (spatial + temporal) $C B C$}

This approach simultaneously involves both benefits of temporal and spatial CBC techniques to scale up the power/energy of the ultrafast fiber lasers. This configuration consists of $\mathrm{N}$ spatially separated amplifier modules (spatial $\mathrm{CBC}$ ), in which there are $\mathrm{M}$ pulse replicas in each amplifier module (temporal CBC). Figure 15 provides the schematic representation of this method. In 2012, the first passive CBC of 8 temporally and spatially separated ultrashort pulses with the pulse energy of $3.1 \mu \mathrm{J}$ was reported by Danialt et al. [303]. Later in 2015, Guichard et al. reported $1.1 \mathrm{~mJ}$ ultrafast pulses via the combination of $\mathrm{CPA}+\mathrm{DPA}$ in a passive CBC context [302].

In the case of active multidimensional configuration, Kienel et al. in a proof-of-principle experiment demonstrated the active multidimensional CBC of ultrashort fiber lasers with the pulse energy of $37 \mu \mathrm{J}$ (without using the CPA technique) [304]. However, during the last years, the multidimensional CBC configuration has experienced rapid development, resulting in a demonstration of a $23 \mathrm{~mJ}$ high-power fiber CPA system in 2019 $[173,175]$.

\subsection{Spectral CBC (spectral pulse synthesis)}

Spectral coherent beam combination (SCBC) refers to the coherent combination of the ultrafast optical pulses with different optical spectrums, in which the combined beam is broader than individual pulses. In this approach, the initial broadband pulse is spectrally split into $\mathrm{N}$ narrow-band pulses. After amplification of each narrowband pulse in

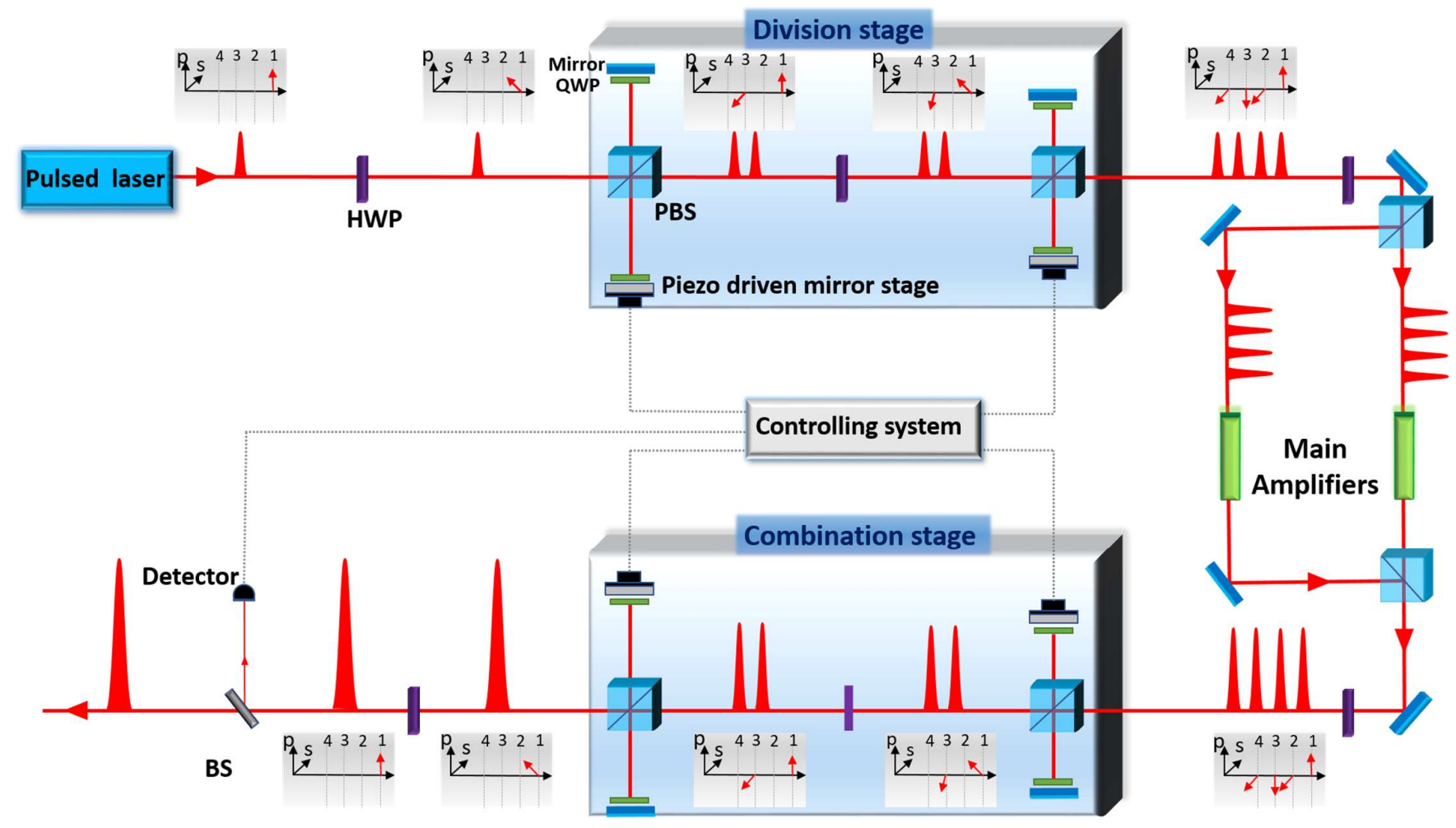

Figure 15. Schematic representation of multidimentional coherent beam combination of ultrafast pulsed laser. 
individual amplifiers, they are combined via spectral combiners (Figure 16). This type of $\mathrm{CBC}$ is a bit deviated in terms of the definition of the CBC concept, in which in CBC of ultrashort pulses it is supposed that all the individual pulses are the same (except polarization state), while in SCBC, pulses have different optical spectra and the combined beam is broader than the individual pulses. Splitting the initial broadband pulse to the N narrowband pulses is performed by the spectral filters, operating as a wavelength-dependent beam splitter. For the spectral combination of the optical pulses, various elements can be utilized, including diffraction grating [305], volume brag grating [306], and multi-layered dielectric filters [307]. The latter is more feasible in the application due to its spatial-dispersion free function, which requires no spatial chirp compensation [307].

The first experimental proof of principle was demonstrated in 2013 by Chang et al., in which three laser channels were combined coherently via a modified LOCSET phase-locking algorithm [308]. In this experiment, the phase errors between spectrally non-overlapping combined signals were measured via two-photon absorption (TPA) detector.

In the same year, Guichard et al. utilized SCBC to overcome the gain narrowing in femtosecond laser pulses through combining two-channel pulse synthesis [309]. In this regard, Rigud et al. proposed a new version of SCBC that was based on amplification on a single multicore fiber [310]. In the work, they designed and utilized a special linear multicore fiber with 12 ytterbium-doped cores. The initial pulse was divided into 12 bands spectral component via a blazed grating and coupled to the linear multi-core fiber via a microlens array for amplification. The microlens array collimates the output beams in the output of the multicore fiber. In the final, combination stage, by implementing a set of a lens and blazed grating, 12 spectrally divided pulses were coherently combined.

However, in contrast to other $\mathrm{CBC}$ techniques for ultrafast pulsed lasers reviewed in this article, the SCBC method has not been well developed yet. Therefore, it requires deep investigation to be suitable for implementation in the high output power/energy pulsed lasers [311].

In conclusion of CBC of ultrafast fiber lasers, the spatial CBC method currently is the most convenient approach for realizing the high average power ultrafast laser systems [169]. On the other hand, multidimensional CBC is more popular for realizing high-energy pulses [175]. It should be highlighted that in realizing the high average power CBC systems, the IBSs are mainly used as combining elements $[169,296]$, while in conducting high energy pulsed lasers, PBSs and thin-film polarizers (TFPs) are commonly utilized [173176]. Table 2 provides the best representatives results of the CBC of ultrafast fiber lasers.

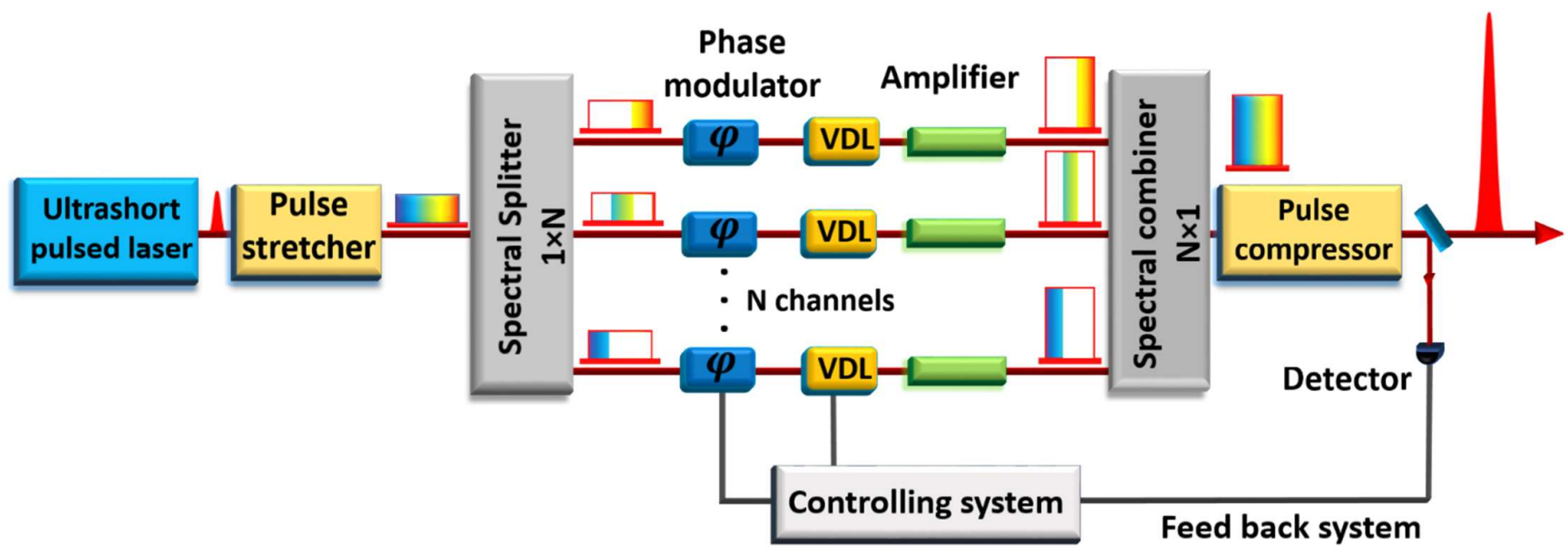

Figure 16. Schematic representation of Spectral coherent beam combination of ultrafast pulsed laser. 


\section{Coherent beam combining of $\mathrm{CW}$ fiber lasers}

In this section, the most prominent representatives of the approaches for realizing a $\mathrm{CBC}$ of $\mathrm{CW}$ fiber lasers will be reviewed in two distinct groups, corresponding to their combining geometry.

\subsection{Tiled aperture}

As discussed before, the tiled aperture is the most feasible geometry for $\mathrm{CBC}$ of fiber lasers, in which the laser beams interfere only in the far-field. This approach has enormous potentials for output power/channel scaling and implementation in directed-energy applications.

- $\quad$ Directed- energy applications

Tiled aperture geometry has considerable potential for direct energy applications, notably for compensating the wavefront distortion induced by atmospheric turbulence [109]. Indeed, by implementing a target-in-the loop CBC system along with adaptive control of piston and tip/tilt phases, efficient laser beam projection in $\mathrm{km}$ range can be achieved. As a case in point, in 2011 the first experimental demonstration of $C B C$ over $7 \mathrm{~km}$ atmospheric horizontal propagation path was reported by Weyrauch et al. [312]. In this experiment, they implemented seven adaptive fiber collimators, with the capability of electronic control of wavefront phase tip and tilts. By implementing the SPGD algorithm in the targetin-the-loop setting, they managed to optimize the phase shift distribution to compensate for the optical wavefront distortion induced by atmospheric turbulence. In an improvement, they experimentally demonstrated efficient adaptive mitigation of the phase aberrations over the same path under the strong scintillation conditions, which compared to their previous work, the number of channels was increased from 7 to 21 channels [166].

- $\quad$ Power scaling

Since the tiled aperture configuration does not require the combining element, there is no power scaling limitation due to the optical damage issues. Indeed, in this method, the optical intensity can be kept below the optical damage threshold. After demonstrating the combinability of a $1.4 \mathrm{~kW}$ fiber laser in 2010, there has been an ongoing effort to realize multi (tens of) $\mathrm{kW} \mathrm{CBC}$ of CW fiber lasers [313]. In 2011, the first $\mathrm{kW}$ level CBC of fiber lasers was demonstrated by Ma et al., using the single-frequency dithering technique [279]. In the same year, a $4 \mathrm{~kW}$ output power fiber laser was achieved through the CBC of eight laser amplifiers, with a combining efficiency of $78 \%$ [147]. In 2020, a $16 \mathrm{~kW}$ single-mode fiber laser with the dynamic beam for advanced material processing was reported, based on the coherent combination of 32 fiber amplifiers in the optical phased array (OPA) technique $[167,314]$.

Recently, a $7.1 \mathrm{~kW} \mathrm{CBC}$ of seven narrow-linewidth, linear-polarized all-fiber amplifiers was achieved by Ma et al. [168]. In that work, they implemented the SPDG algorithm for the phase-locking system, and by applying the 95\% filling factor, they achieved $86 \%$ contrast of the far-field intensity pattern.

\subsection{Filled aperture}

The tiled aperture combining geometry has an inherent drawback due to the emerging of side lobes, leading to the beam quality and combining efficiency degradation. Hence, filled aperture combining techniques have received great attention. However, despite the tiled aperture combining geometry in which the beams are combined in the farfield (due to propagation), the filled aperture technique needs the optical combining elements to overlap the beams in the near field. In this regard, various optical elements have been implemented, including DOE, IBS, PBS, re-imaging waveguide (RIW), high power fiber couplers (HPFC), and all-fiber-based photonic lantern (AFPL) [315]. Among them, a $\mathrm{CBC}$ system based on the DOE has provided the output power world record for $\mathrm{CBC}$ of CW fiber lasers in filled aperture geometry [181]. Table 3 provides the best representatives results of the $\mathrm{CBC}$ of $\mathrm{CW}$ fiber lasers. 
Table 2. Representative results of CBC of ultrafast fiber lasers.

\begin{tabular}{|c|c|c|c|c|c|c|c|c|c|c|c|}
\hline $\begin{array}{l}\text { Combining } \\
\text { configura- } \\
\text { tion }\end{array}$ & 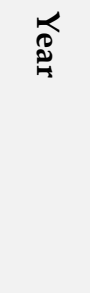 & 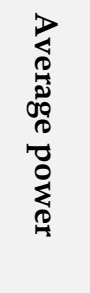 & 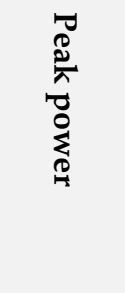 & 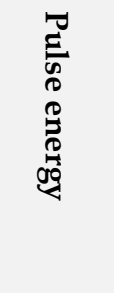 & 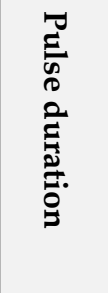 & 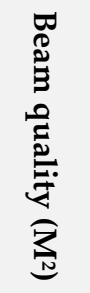 & 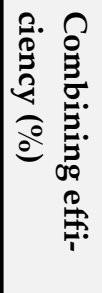 & 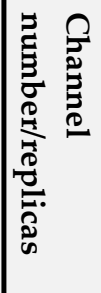 & 串 & 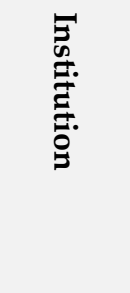 & 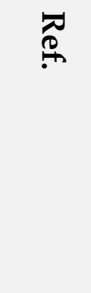 \\
\hline \multirow{3}{*}{ Spatial } & 2014 & $\begin{array}{l}230 \\
W\end{array}$ & $22 \mathrm{GW}$ & $\begin{array}{l}5.7 \\
\mathrm{~mJ}\end{array}$ & $200 \mathrm{fs}$ & $\leq 1.3$ & 88 & 4 & $\begin{array}{l}\text { CPA, HC } \\
\text { detection, } \\
\text { PBS } \\
\end{array}$ & Jena & [16] \\
\hline & 2020 & $\begin{array}{l}10.4 \\
\mathrm{~kW}\end{array}$ & $0.5 \mathrm{GW}$ & $130 \mu \mathrm{J}$ & $254 \mathrm{fs}$ & $\leq 1.2$ & 96 & 12 & $\begin{array}{l}\text { CPA, } \\
\text { LOCSET, IBS }\end{array}$ & Jena & [169] \\
\hline & 2021 & $\begin{array}{c}1 \\
\mathrm{~kW}\end{array}$ & $68 \mathrm{GW}$ & $10 \mathrm{~mJ}$ & $120 \mathrm{fs}$ & $\leq 1.2$ & 94 & 16 & $\begin{array}{l}\text { CPA, HC } \\
\text { detection, } \\
\text { PBS \& TFP }\end{array}$ & Jena & [176] \\
\hline \multirow{2}{*}{ Temporal } & 2013 & $77 \mathrm{~W}$ & $1.3 \mathrm{GW}$ & $430 \mu \mathrm{J}$ & $320 \mathrm{fs}$ & $\leq 1.3$ & 97 & 2 & $\begin{array}{l}\text { CPA+DPA, } \\
\text { TFP, } \\
\text { passive }\end{array}$ & $\begin{array}{l}\text { Ampli- } \\
\text { tude } \\
\text { \&CNRS }\end{array}$ & [300] \\
\hline & 2014 & $\begin{array}{c}37.5 \\
W\end{array}$ & $2.9 \mathrm{GW}$ & $\begin{array}{c}1.25 \\
\mathrm{~mJ}\end{array}$ & $380 \mathrm{fs}$ & - & 75 & 4 & $\begin{array}{l}\text { DPA, } \\
\text { LOCSET, } \\
\text { PBS }\end{array}$ & Jena & [301] \\
\hline \multirow{4}{*}{$\begin{array}{l}\text { Spatio- } \\
\text { temporal }\end{array}$} & 2015 & $55 \mathrm{~W}$ & $3.1 \mathrm{GW}$ & $1.1 \mathrm{~mJ}$ & $300 \mathrm{fs}$ & $\leq 1.3$ & 90 & $2 \times 2$ & $\begin{array}{l}\mathrm{CPA}+\mathrm{DPA} \\
\text { passive }\end{array}$ & $\begin{array}{l}\text { Ampli- } \\
\text { tude } \\
\text { \&CNRS }\end{array}$ & [302] \\
\hline & 2015 & - & - & $37 \mu \mathrm{J}$ & $50 \mathrm{ps}$ & - & 75 & $2 \times 4$ & $\begin{array}{l}\text { DPA, } \\
\text { LOCSET, } \\
\text { PBS } \\
\end{array}$ & Jena & [304] \\
\hline & 2016 & $\begin{array}{c}700 \\
W\end{array}$ & $45 \mathrm{GW}$ & $12 \mathrm{~mJ}$ & $262 \mathrm{fs}$ & $\leq 1.2$ & 78 & $8 \times 4$ & $\begin{array}{l}\text { CPA+DPA, } \\
\text { LOCSET, } \\
\text { PBS \&TFP }\end{array}$ & Jena & [173] \\
\hline & 2019 & $\begin{array}{c}674 \\
W\end{array}$ & $80 \mathrm{GW}$ & $23 \mathrm{~mJ}$ & $235 \mathrm{fs}$ & - & 71 & $12 \times 8$ & $\begin{array}{l}\text { CPA+DPA, } \\
\text { LOCSET, } \\
\text { PBS \& TFP }\end{array}$ & Jena & [175] \\
\hline \multirow{3}{*}{$\begin{array}{l}\text { spectral } \\
\text { synthesis }\end{array}$} & 2013 & $\begin{array}{c}273 \\
\mathrm{~mW}\end{array}$ & - & - & $403 \mathrm{fs}$ & - & 85.8 & 3 & $\begin{array}{l}\text { Spectral fil- } \\
\text { ters, } \\
\text { LOCSET }\end{array}$ & UMich & [308] \\
\hline & 2013 & $10 \mathrm{~W}$ & $2 \mathrm{MW}$ & $0.29 \mu \mathrm{J}$ & $130 \mathrm{fs}$ & $\leq 1.4$ & 86 & 3 & $\begin{array}{l}\text { LMA fiber, } \\
\text { dichroic } \\
\text { mirror }\end{array}$ & CNRS & [309] \\
\hline & 2013 & $\begin{array}{l}370 \\
\mathrm{~mW}\end{array}$ & - & - & $290 \mathrm{fs}$ & - & - & 12 & $\begin{array}{l}\text { MCF, } \\
\text { Grating } \\
\text { and MLA } \\
\text { twin pulses } \\
\text { with 1.75ps } \\
\text { separation }\end{array}$ & CNRS & [310] \\
\hline
\end{tabular}


Table 3. Representative results of CBC of CW fiber lasers.

\begin{tabular}{|c|c|c|c|c|c|c|c|c|}
\hline \multirow[t]{8}{*}{$\begin{array}{c}\text { Tiled } \\
\text { aperture }\end{array}$} & $\begin{array}{c}\text { Directed } \\
\text { energy }\end{array}$ & Year & Distance & $\begin{array}{l}\text { Channel } \\
\text { number }\end{array}$ & $\begin{array}{l}\text { Tip/tilt } \\
\text { correction }\end{array}$ & $\begin{array}{l}\text { phase control } \\
\text { method }\end{array}$ & Institution & Ref. \\
\hline & & 2011 & $7 \mathrm{~km}$ & 7 & $\sqrt{ }$ & SPGD & UD & [312] \\
\hline & & 2015 & $7 \mathrm{~km}$ & 21 & $\sqrt{ }$ & SPGD & UD & [149] \\
\hline & Power scaling & Year & $\begin{array}{l}\text { power } \\
(\mathrm{kW})\end{array}$ & $\begin{array}{l}\text { Channel } \\
\text { number }\end{array}$ & $\begin{array}{l}\text { Combining } \\
\text { efficiency } \\
(\%)\end{array}$ & $\begin{array}{l}\text { phase control } \\
\text { method }\end{array}$ & Institution & Ref. \\
\hline & & 2011 & 1.08 & 9 & $85^{*}$ & SFD & NUDT & [279] \\
\hline & & 2011 & 4 & 8 & 78 & SPGD & MIT & [147] \\
\hline & & 2020 & 16 & 32 & $>95$ & OPA & CIVAN & [316] \\
\hline & & 2021 & 7.1 & 7 & $86^{*}$ & SPGD & NUDT & [168] \\
\hline \multirow[t]{5}{*}{$\begin{array}{c}\text { Filled } \\
\text { aperture }\end{array}$} & $\begin{array}{l}\text { Combining } \\
\text { technique }\end{array}$ & Year & $\begin{array}{l}\text { Power } \\
(\mathrm{kW})\end{array}$ & $\begin{array}{l}\text { Channel } \\
\text { number }\end{array}$ & $\begin{array}{l}\text { Combining } \\
\text { efficiency } \\
(\%)\end{array}$ & $\begin{array}{l}\text { phase control } \\
\text { method }\end{array}$ & Institution & Ref. \\
\hline & DOE & 2016 & 5 & 5 & 82 & LOCSET & AFRL & [181] \\
\hline & PBS & 2017 & 2.16 & 4 & 94.5 & & NUDT & [108] \\
\hline & AFPL & 2017 & 1.27 & 3 & - & SPGD & MIT & [315] \\
\hline & RIW & 2010 & 0.1 & 4 & 80 & LOCSET & LM Corp & [317] \\
\hline
\end{tabular}

*. The fringe contrast of the far-field intensity pattern

\section{Conclusion}

In this paper, the coherent beam combining of fiber lasers from basic concepts to the recent development in both continuous wave and pulsed regimes is reviewed. CBC is a promising, versatile technique for scaling the power/energy of lasers far beyond what it is possible to obtain from a single laser, realizing the hundred $\mathrm{kW}$ level in average power and joule-class pulse energy. CBC can open up unprecedented new possibilities in science, industry, and defence. For example, this technique can provide the energy level needed in extreme ultraviolet (EUV) applications (such as second-harmonic generators), or in particle physics as laser-based particle accelerators. In industrial applications, both coherently combined CW and pulsed lasers are implemented for advanced material processing such as high-speed ultrafine cutting and welding. In defence applications, coherently combined laser systems are used as directed-energy weapons.

Coherent beam combing technology is also the key enabler in realizing ultra-intense laser pulses and high-power laser systems. For example, the extreme light infrastructure (ELI) project, aiming to investigate the light-matter interactions at high laser intensities and on ultrashort timescales is based on CBC technology [318].

While technological advancements have made it technically possible to achieve 10$100 \mathrm{~kW} \mathrm{CBC} \mathrm{systems,} \mathrm{a} \mathrm{major} \mathrm{hurdle} \mathrm{in} \mathrm{these} \mathrm{systems} \mathrm{is} \mathrm{still} \mathrm{the} \mathrm{complexity} \mathrm{and} \mathrm{increased}$ footprint required for all the combining elements. In relation to this, the system cost is not only driven by the cost of the optical power, as phase controls, delay controls and related feedback loops need to be incorporated for each channel. The cost of these additional parts can end up being several thousand to tens of thousands of USD per channel, obviously largely depending on the chosen geometry.

In the context of passive and active phase-locking systems, the latter provides more straightforward scaling-up capabilities. In the CW regime, tiled aperture geometry has enormous potential to scale up the power with the near diffraction-limited beam quality, and it is considered as the best approach for directed-energy applications. In terms of pulsed regime, the highest power/energy records of $\mathrm{CBC}$ laser systems have been 
demonstrated in the filled aperture geometry. To date, the records of few tens of mJ pulse energy, few tens of GW peak power and few tens of $\mathrm{kW}$ average output power were experimentally realized through the $\mathrm{CBC}$ of multiple fiber lasers.

Acknowledgements: The work is part of the Academy of Finland Flagship Programme, Photonics Research and Innovation (PREIN), decision 320165.

Funding: This work were supported by: i) the PULSE project, grant agreement number 824996, funded by the European Commission Horizon 2020 Program, ii) Finnish Cultural Foundation, grant number: 00210294, and iii) Finnish National Agency for Education (EDUFI) Fellowship 2020, grant number: TM-20-11453.

\section{References}

1. Zervas, M.N.; Codemard, C.A. High Power Fiber Lasers: A Review. IEEE J. Sel. Top. Quantum Electron. 2014, 20, 219-241, doi:10.1109/JSTQE.2014.2321279.

2. Moulton, P.F.; Rines, G.A.; Slobodtchikov, E. V.; Wall, K.F.; Frith, G.; Samson, B.; Carter, A.L.G. Tm-Doped Fiber Lasers: Fundamentals and Power Scaling. IEEE J. Sel. Top. Quantum Electron. 2009, 15, 85-92, doi:10.1109/JSTQE.2008.2010719.

3. Richardson, D.J.; Nilsson, J.; Clarkson, W.A. High power fiber lasers: current status and future perspectives [Invited]. J. Opt. Soc. Am. B 2010, 27, B63, doi:10.1364/JOSAB.27.000B63.

4. Jauregui, C.; Limpert, J.; Tünnermann, A. High-power fibre lasers. Nat. Photonics 2013, 7, 861-867, doi:10.1038/nphoton.2013.273.

5. $\quad$ Fang, Q.; Li, J.; Shi, W.; Qin, Y.; Xu, Y.; Meng, X.; Norwood, R.A.; Peyghambarian, N. 5 kW near-Diffraction-limited and 8 kW High-Brightness Monolithic Continuous Wave Fiber lasers Directly Pumped by laser Diodes. IEEE Photonics J. 2017, 9, doi:10.1109/JPHOT.2017.2744803.

6. Liu, Z.; Jin, X.; Su, R.; Ma, P.; Zhou, P. Development status of high power fiber lasers and their coherent beam combination. Sci. China Inf. Sci. 2019, 62, 41301, doi:10.1007/s11432-018-9742-0.

7. Chang, G.; Wei, Z. Ultrafast Fiber Lasers: An Expanding Versatile Toolbox. iScience 2020, 23, 101101, doi:10.1016/j.isci.2020.101101.

8. Fermann, M.E.; Hartl, I. Ultrafast Fiber Laser Technology. IEEE J. Sel. Top. Quantum Electron. 2009, 15, 191-206, doi:10.1109/JSTQE.2008.2010246.

9. Jun Jun Lim; Sujecki, S.; Lei Lang; Zhichao Zhang; Paboeuf, D.; Pauliat, G.; Lucas-Leclin, G.; Georges, P.; MacKenzie, R.; Bream, P.; et al. Design and Simulation of Next-Generation High-Power, High-Brightness Laser Diodes. IEEE J. Sel. Top. Quantum Electron. 2009, 15, 993-1008, doi:10.1109/JSTQE.2008.2011286.

10. Snitzer, E.; Po, H.; Hakimi, F.; Tumminelli, R.; McCollum, B.C. DOUBLE CLAD, OFFSET CORE Nd FIBER LASER. In Proceedings of the Optical Fiber Sensors; OSA: Washington, D.C., 1988; p. PD5.

11. Yang, B.; Wang, P.; Zhang, H.; Xi, X.; Shi, C.; Wang, X.; Xu, X. 6 kW single mode monolithic fiber laser enabled by effective mitigation of the transverse mode instability. Opt. Express 2021, 29, 26366, doi:10.1364/OE.433630.

12. Shcherbakov, E.A.; Fomin, V. V.; Abramov, A.A.; Ferin, A.A.; Mochalov, D. V.; Gapontsev, V.P. Industrial grade 100 kW power CW fiber laser. In Proceedings of the Advanced Solid-State Lasers Congress; OSA: Washington, D.C., 2013; Vol. 5, p. ATh4A.2.

13. Shiner, B. The Impact of Fiber Laser Technology on the World Wide Material Processing Market. In Proceedings of the CLEO: 
2013; OSA: Washington, D.C., 2013; p. AF2J.1.

14. Strickland, D.; Mourou, G. Compression of amplified chirped optical pulses. Opt. Commun. 1985, 56, 219-221, doi:10.1016/0030-4018(85)90120-8.

15. Eidam, T.; Hanf, S.; Seise, E.; Andersen, T. V.; Gabler, T.; Wirth, C.; Schreiber, T.; Limpert, J.; Tünnermann, A. Femtosecond fiber CPA system emitting $830 \mathrm{~W}$ average output power. Opt. Lett. 2010, 35, 94, doi:10.1364/OL.35.000094.

16. Klenke, A.; Hädrich, S.; Eidam, T.; Rothhardt, J.; Kienel, M.; Demmler, S.; Gottschall, T.; Limpert, J.; Tünnermann, A. 22 GW peak-power fiber chirped-pulse-amplification system. Opt. Lett. 2014, 39, 6875, doi:10.1364/OL.39.006875.

17. Dawson, J.W.; Messerly, M.J.; Beach, R.J.; Shverdin, M.Y.; Stappaerts, E.A.; Sridharan, A.K.; Pax, P.H.; Heebner, J.E.; Siders, C.W.; Barty, C.P.J. Analysis of the scalability of diffraction-limited fiber lasers and amplifiers to high average power. Opt. Express 2008, 16, 13240, doi:10.1364/OE.16.013240.

18. Zervas, M.N. Power scaling limits in high power fiber amplifiers due to transverse mode instability, thermal lensing, and fiber mechanical reliability. In Proceedings of the Fiber Lasers XV: Technology and Systems; Carter, A.L., Hartl, I., Eds.; SPIE, 2018; p. 4.

19. Zhu, J.; Zhou, P.; Ma, Y.; Xu, X.; Liu, Z. Power scaling analysis of tandem-pumped Yb-doped fiber lasers and amplifiers. Opt. Express 2011, 19, 18645, doi:10.1364/OE.19.018645.

20. Zervas, M.N. Transverse mode instability, thermal lensing and power scaling in $\mathrm{Yb} 3+$-doped high-power fiber amplifiers. Opt. Express 2019, 27, 19019, doi:10.1364/OE.27.019019.

21. Agrawal, G. Nonlinear Fiber Optics - 4th Edition; 2006; ISBN 9780123695161.

22. Boyd, R.W. Nonlinear Optics; Academic Press, 2020; ISBN 9780128110034.

23. Jauregui, C.; Stihler, C.; Limpert, J. Transverse mode instability. Adv. Opt. Photonics 2020, 12, 429, doi:10.1364/AOP.385184.

24. Hejaz, K.; Shayganmanesh, M.; Rezaei-Nasirabad, R.; Roohforouz, A.; Azizi, S.; Abedinajafi, A.; Vatani, V. Modal instability induced by stimulated Raman scattering in high-power Yb-doped fiber amplifiers. Opt. Lett. 2017, 42, 5274, doi:10.1364/OL.42.005274.

25. Li, Z.; Jing, F.; Li, C.; Liu, Y.; Luo, Q.; Lin, H.; Huang, Z.; Xu, S.; Yang, Z.; Wang, J. Impact of Stimulated Raman Scattering on the Transverse Mode Instability Threshold. IEEE Photonics J. 2018, 10, 1-9, doi:10.1109/JPHOT.2018.2842467.

26. Kim, J.; Dupriez, P.; Codemard, C.; Nilsson, J.; Sahu, J.K. Suppression of stimulated Raman scattering in a high power Ybdoped fiber amplifier using a W-type core with fundamental mode cut-off. Opt. Express 2006, 14, 5103, doi:10.1364/OE.14.005103.

27. Jansen, F.; Nodop, D.; Jauregui, C.; Limpert, J.; Tünnermann, A. Modeling the inhibition of stimulated Raman scattering in passive and active fibers by lumped spectral filters in high power fiber laser systems. Opt. Express 2009, 17, 16255, doi:10.1364/OE.17.016255.

28. Xu, H.; Jiang, M.; Shi, C.; Zhou, P.; Zhao, G.; Gu, X. Spectral shaping for suppressing stimulated-Raman-scattering in a fiber laser. Appl. Opt. 2017, 56, 3538, doi:10.1364/AO.56.003538.

29. Shi, C.; Su, R.T.; Zhang, H.W.; Yang, B.L.; Wang, X.L.; Zhou, P.; Xu, X.J.; Lu, Q.S. Experimental Study of Output Characteristics of Bi-Directional Pumping High Power Fiber Amplifier in Different Pumping Schemes. IEEE Photonics J. 2017, 9, 1-10, doi:10.1109/JPHOT.2017.2679753.

30. Zenteno, L.A.; Wang, J.; Walton, D.T.; Ruffin, B.A.; Li, M.J.; Gray, S.; Crowley, A.; Chen, X. Suppression of Raman gain in single-transverse-mode dual-hole-assisted fiber. Opt. Express 2005, 13, 8921, doi:10.1364/OPEX.13.008921.

31. Liu, A.; Chen, X.; Li, M.-J.; Wang, J.; Walton, D.T.; Zenteno, L.A. Suppressing nonlinear effects for power scaling of high power fiber lasers. Passiv. Components Fiber-based Devices IV 2007, 6781, 67810H, doi:10.1117/12.745958.

32. Li, T.; Ke, W.; Ma, Y.; Sun, Y.; Gao, Q. Suppression of stimulated Raman scattering in a high-power fiber amplifier by inserting long transmission fibers in a seed laser. J. Opt. Soc. Am. B 2019, 36, 1457, doi:10.1364/JOSAB.36.001457. 
33. Jiao, K.; Shen, H.; Guan, Z.; Yang, F.; Zhu, R. Suppressing stimulated Raman scattering in kW-level continuous-wave MOPA fiber laser based on long-period fiber gratings. Opt. Express 2020, 28, 6048, doi:10.1364/OE.384760.

34. Hadjifotiou, A.; Hill, G.A. Suppression of stimulated brillouin backscattering by psk modulation for high-power optical transmission. IEE Proc. J Optoelectron. 1986, 133, 256, doi:10.1049/ip-j.1986.0041.

35. Willems, F.W.; Muys, W.; Leong, J.S. Simultaneous suppression of stimulated Brillouin scattering and interferometric noise in externally modulated lightwave AM-SCM systems. IEEE Photonics Technol. Lett. 1994, 6, 1476-1478, doi:10.1109/68.392206.

36. Broderick, N.G.R.; Offerhaus, H.L.; Richardson, D.J.; Sammut, R.A.; Caplen, J.; Dong, L. Large Mode Area Fibers for High Power Applications. Opt. Fiber Technol. 1999, 5, 185-196, doi:10.1006/ofte.1998.0292.

37. Koyamada, Y.; Sato, S.; Nakamura, S.; Sotobayashi, H.; Chujo, W. Simulating and designing Brillouin gain spectrum in singlemode fibers. J. Light. Technol. 2004, 22, 631-639, doi:10.1109/JLT.2003.822007.

38. Lee, J.; Lee, K.H.; Jeong, H.; Park, M.; Seung, J.H.; Lee, J.H. 2.05 kW all-fiber high-beam-quality fiber amplifier with stimulated Brillouin scattering suppression incorporating a narrow-linewidth fiber-Bragg-grating-stabilized laser diode seed source. Appl. Opt. 2019, 58, 6251, doi:10.1364/AO.58.006251.

39. Tian, X.; Zhao, X.; Wang, M.; Wang, Z. Suppression of stimulated Brillouin scattering in optical fibers by tilted fiber Bragg gratings. Opt. Lett. 2020, 45, 4802, doi:10.1364/OL.401433.

40. Prakash, R.; Vikram, B.S.; Supradeepa, V.R. Polarization maintaining, narrow linewidth fiber laser with \&gt;1kW output power using a novel dual sine and noise modulation for enhanced SBS suppression. In Proceedings of the Fiber Lasers XVIII: Technology and Systems; Zervas, M.N., Jauregui-Misas, C., Eds.; SPIE, 2021; Vol. 11665, p. 3.

41. Anderson, B.M.; MacDonald, K.; Taliaferro, A.; Flores, A. SBS Suppression techniques in high-power, narrow-linewidth fiber amplifiers. In Proceedings of the Fiber Lasers XVIII: Technology and Systems; Zervas, M.N., Jauregui-Misas, C., Eds.; SPIE, 2021; Vol. 11665, p. 12.

42. Zeringue, C.; Dajani, I.; Naderi, S.; Moore, G.T.; Robin, C. A theoretical study of transient stimulated Brillouin scattering in optical fibers seeded with phase-modulated light. Opt. Express 2012, 20, 21196, doi:10.1364/OE.20.021196.

43. Flores, A.; Robin, C.; Lanari, A.; Dajani, I. Pseudo-random binary sequence phase modulation for narrow linewidth, kilowatt, monolithic fiber amplifiers. Opt. Express 2014, 22, 17735, doi:10.1364/OE.22.017735.

44. Yang, Y.; Li, B.; Liu, M.; Huang, X.; Feng, Y.; Cheng, D.; He, B.; Zhou, J.; Nilsson, J. Optimization and visualization of phase modulation with filtered and amplified maximal-length sequence for SBS suppression in a short fiber system: a theoretical treatment. Opt. Express 2021, 29, 16781, doi:10.1364/OE.426070.

45. Beier, F.; Hupel, C.; Kuhn, S.; Hein, S.; Nold, J.; Proske, F.; Sattler, B.; Liem, A.; Jauregui, C.; Limpert, J.; et al. Single mode 43 kW output power from a diode-pumped Yb-doped fiber amplifier. Opt. Express 2017, 25, 14892, doi:10.1364/OE.25.014892.

46. Kobyakov, A.; Kumar, S.; Chowdhury, D.Q.; Ruffin, A.B.; Sauer, M.; Bickham, S.R.; Mishra, R. Design concept for optical fibers with enhanced SBS threshold. Opt. Express 2005, 13, 5338, doi:10.1364/OPEX.13.005338.

47. Ruffin, A.B.; Li, M.-J.; Chen, X.; Kobyakov, A.; Annunziata, F. Brillouin gain analysis for fibers with different refractive indices. Opt. Lett. 2005, 30, 3123, doi:10.1364/OL.30.003123.

48. Fini, J.M. Bend-resistant design of conventional and microstructure fibers with very large mode area. Opt. Express 2006, 14, 69, doi:10.1364/OPEX.14.000069.

49. Liu, A. Novel SBS suppression scheme for high-power fiber amplifiers. In Proceedings of the Fiber Lasers III: Technology, Systems, and Applications; SPIE, 2006; Vol. 6102, p. 61021R.

50. Li, M.-J.; Chen, X.; Wang, J.; Gray, S.; Liu, A.; Demeritt, J.A.; Ruffin, A.B.; Crowley, A.M.; Walton, D.T.; Zenteno, L.A. Al/Ge co-doped large mode area fiber with high SBS threshold. Opt. Express 2007, 15, 8290, doi:10.1364/oe.15.008290.

51. Mermelstein, M.D.; Andrejco, M.J.; Fini, J.; Yablon, A.; Headley III, C.; DiGiovanni, D.J.; McCurdy, A.H. 11.2 dB SBS gain suppression in a large mode area Yb-doped optical fiber. In Proceedings of the Fiber Lasers V: Technology, Systems, and 
Applications; Broeng, J., Headley III, C., Eds.; SPIE, 2008; Vol. 6873, p. 68730N.

52. Harish, A.V.; Nilsson, J. Suppression of Stimulated Brillouin Scattering in Single-Frequency Fiber Raman Amplifier Through Pump Modulation. J. Light. Technol. 2019, 37, 3280-3289, doi:10.1109/JLT.2019.2914081.

53. Huang, Y.; Yan, P.; Wang, Z.; Tian, J.; Li, D.; Xiao, Q.; Gong, M. 2.19 kW narrow linewidth FBG-based MOPA configuration fiber laser. Opt. Express 2019, 27, 3136, doi:10.1364/OE.27.003136.

54. Wang, Y.; Feng, Y.; Ma, Y.; Chang, Z.; Peng, W.; Sun, Y.; Gao, Q.; Zhu, R.; Tang, C. 2.5 kW Narrow Linewidth Linearly Polarized All-Fiber MOPA With Cascaded Phase-Modulation to Suppress SBS Induced Self-Pulsing. IEEE Photonics J. 2020, 12, 1-15, doi:10.1109/JPHOT.2020.2997935.

55. Wang, Y.; Ke, W.; Peng, W.; Chang, Z.; Feng, Y.; Sun, Y.; Gao, Q.; Ma, Y.; Zhu, R.; Tang, C. 3 kW, 0.2 nm narrow linewidth linearly polarized all-fiber laser based on a compact MOPA structure. Laser Phys. Lett. 2020, 17, 075101, doi:10.1088/1612202X/ab8e42.

56. Lin, H.; Tao, R.; Li, C.; Wang, B.; Guo, C.; Shu, Q.; Zhao, P.; Xu, L.; Wang, J.; Jing, F.; et al. 3.7 kW monolithic narrow linewidth single mode fiber laser through simultaneously suppressing nonlinear effects and mode instability. Opt. Express 2019, 27, 9716, doi:10.1364/OE.27.009716.

57. Stolen, R.H.; Lin, C. Self-phase-modulation in silica optical fibers. Phys. Rev. A 1978, 17, 1448-1453, doi:10.1103/PhysRevA.17.1448.

58. Shimizu, F. Frequency Broadening in Liquids by a Short Light Pulse. Phys. Rev. Lett. 1967, 19, 1097-1100, doi:10.1103/PhysRevLett.19.1097.

59. Hasegawa, A.; Tappert, F. Transmission of stationary nonlinear optical pulses in dispersive dielectric fibers. I. Anomalous dispersion. Appl. Phys. Lett. 1973, 23, 142-144, doi:10.1063/1.1654836.

60. Farrow, R.L.; Kliner, D.A. V.; Hadley, G.R.; Smith, A. V. Peak-power limits on fiber amplifiers imposed by self-focusing. Opt. Lett. 2006, 31, 3423, doi:10.1364/OL.31.003423.

61. Dong, L. Approximate Treatment of the Nonlinear Waveguide Equation in the Regime of Nonlinear Self-Focus. J. Light. Technol. 2008, 26, 3476-3485, doi:10.1109/JLT.2008.925685.

62. Schimpf, D.N.; Eidam, T.; Seise, E.; Hädrich, S.; Limpert, J.; Tünnermann, A. Circular versus linear polarization in laseramplifiers with Kerr-nonlinearity. Opt. Express 2009, 17, 18774, doi:10.1364/OE.17.018774.

63. Smith, A. V.; Do, B.T.; Hadley, G.R.; Farrow, R.L. Optical Damage Limits to Pulse Energy From Fibers. IEEE J. Sel. Top. Quantum Electron. 2009, 15, 153-158, doi:10.1109/JSTQE.2008.2010331.

64. Zheng, J.; Zhao, W.; Zhao, B.; Li, Z.; Li, G.; Gao, Q.; Ju, P.; Gao, W.; She, S.; Wu, P. Four-wave mixing effect on high-power continuous-wave all-fiber lasers. Mod. Phys. Lett. B 2018, 32, 1850275, doi:10.1142/S0217984918502755.

65. Eidam, T.; Wirth, C.; Jauregui, C.; Stutzki, F.; Jansen, F.; Otto, H.-J.; Schmidt, O.; Schreiber, T.; Limpert, J.; Tünnermann, A. Experimental observations of the threshold-like onset of mode instabilities in high power fiber amplifiers. Opt. Express 2011, 19, 13218, doi:10.1364/OE.19.013218.

66. Smith, A. V.; Smith, J.J. Influence of pump and seed modulation on the mode instability thresholds of fiber amplifiers. Opt. Express 2012, 20, 24545, doi:10.1364/OE.20.024545.

67. Smith, A. V.; Smith, J.J. Mode instability in high power fiber amplifiers. Opt. Express 2011, 19, 10180, doi:10.1364/OE.19.010180.

68. Jauregui, C.; Eidam, T.; Otto, H.-J.; Stutzki, F.; Jansen, F.; Limpert, J.; Tünnermann, A. Physical origin of mode instabilities in high-power fiber laser systems. Opt. Express 2012, 20, 12912, doi:10.1364/OE.20.012912.

69. Ward, B.; Robin, C.; Dajani, I. Origin of thermal modal instabilities in large mode area fiber amplifiers. Opt. Express 2012, 20, 11407, doi:10.1364/OE.20.011407.

70. Hejaz, K.; Shayganmanesh, M.; Azizi, S.; Abedinajafi, A.; Roohforouz, A.; Rezaei-Nasirabad, R.; Vatani, V. Transverse mode instability of fiber oscillators in comparison with fiber amplifiers. Laser Phys. Lett. 2018, 15, doi:10.1088/1612-202X/aab5f1. 
71. Roohforouz, A.; Eyni Chenar, R.; Rezaei-Nasirabad, R.; Azizi, S.; Hejaz, K.; Hamedani Golshan, A.; Abedinajafi, A.; Vatani, V.; Nabavi, S.H. The effect of population inversion saturation on the transverse mode instability threshold in high power fiber laser oscillators. Sci. Rep. 2021, 11, 21116, doi:10.1038/s41598-021-00400-9.

72. Ke, W.-W.; Wang, X.-J.; Bao, X.-F.; Shu, X.-J. Thermally induced mode distortion and its limit to power scaling of fiber lasers. Opt. Express 2013, 21, 14272, doi:10.1364/OE.21.014272.

73. Zervas, M.N. Power scalability in high power fibre amplifiers. In Proceedings of the 2017 Conference on Lasers and ElectroOptics Europe \& European Quantum Electronics Conference (CLEO/Europe-EQEC); IEEE, 2017; Vol. Part F82-C, pp. 1-1.

74. Brown, D.C.; Hoffman, H.J. Thermal, stress, and thermo-optic effects in high average power double-clad silica fiber lasers. IEEE J. Quantum Electron. 2001, 37, 207-217, doi:10.1109/3.903070.

75. Lapointe, M.-A.; Chatigny, S.; Piché, M.; Cain-Skaff, M.; Maran, J.-N. Thermal effects in high-power CW fiber lasers. In Proceedings of the Fiber Lasers VI: Technology, Systems, and Applications; Gapontsev, D. V., Kliner, D.A., Dawson, J.W., Tankala, K., Eds.; 2009; Vol. 7195, p. 71951U.

76. Stuart, B.C.; Feit, M.D.; Rubenchik, A.M.; Shore, B.W.; Perry, M.D. Laser-Induced Damage in Dielectrics with Nanosecond to Subpicosecond Pulses. Phys. Rev. Lett. 1995, 74, 2248-2251, doi:10.1103/PhysRevLett.74.2248.

77. Smith, A.; Do, B.; Soderlund, M. Deterministic nanosecond laser-induced breakdown thresholds in pure and $\mathrm{Yb} 3+\mathrm{doped}$ fused silica . Fiber Lasers IV Technol. Syst. Appl. 2007, 6453, 645317, doi:10.1117/12.701399.

78. Gapontsev, V.; Gapontsev, D.; Platonov, N.; Shkurikhin, O.; Fomin, V.; Mashkin, A.; Abramov, M.; Ferin, S. 2 kW CW ytterbium fiber laser with record diffraction-limited brightness. In Proceedings of the CLEO/Europe. 2005 Conference on Lasers and Electro-Optics Europe, 2005.; IEEE, 2005; Vol. 12, pp. 508-508.

79. Jain, D.; Jung, Y.; Barua, P.; Alam, S.; Sahu, J.K. Demonstration of ultra-low NA rare-earth doped step index fiber for applications in high power fiber lasers. Opt. Express 2015, 23, 7407, doi:10.1364/OE.23.007407.

80. Midilli, Y.; Ortaç, B. An all-fiber ultra-low numerical aperture high power fiber MOPA system with an output power above 500 W. Opt. InfoBase Conf. Pap. 2019, Part F140-, 14892.

81. Lim, K.-J.; Kai-Wen Seah, S.; Yong'En Ye, J.; Lim, W.W.; Seah, C.-P.; Tan, Y.-B.; Tan, S.; Lim, H.; Sidharthan, R.; Prasadh, A.R.; et al. High absorption large-mode area step-index fiber for tandem-pumped high-brightness high-power lasers. Photonics Res. 2020, 8, 1599, doi:10.1364/PRJ.400755.

82. Paschotta, R.; Nilsson, J.; Barber, P.R.; Caplen, J.E.; Tropper, A.C.; Hanna, D.C. Lifetime quenching in Yb-doped fibres. Opt. Commun. 1997, 136, 375-378, doi:10.1016/S0030-4018(96)00720-1.

83. Huang, L.; Yao, T.; Leng, J.; Guo, S.; Tao, R.; Zhou, P.; Cheng, X. Mode instability dynamics in high-power low-numericalaperture step-index fiber amplifier. Appl. Opt. 2017, 56, 5412, doi:10.1364/AO.56.005412.

84. Midilli, Y.; Ortac, B. Demonstration of an All-Fiber Ultra-Low Numerical Aperture Ytterbium-Doped Large Mode Area Fiber in a Master Oscillator Power Amplifier Configuration Above 1 kW Power Level. J. Light. Technol. 2020, 38, 1915-1920, doi:10.1109/JLT.2020.2970213.

85. Lin, H.; Xu, L.; Li, C.; Shu, Q.; Chu, Q.; Xie, L.; Guo, C.; Zhao, P.; Li, Z.; Wang, J.; et al. 10.6 kW high-brightness cascade-endpumped monolithic fiber lasers directly pumped by laser diodes in step-index large mode area double cladding fiber. Results Phys. 2019, 14, 102479, doi:10.1016/j.rinp.2019.102479.

86. Codemard, C.A.; Sahu, J.K.; Nilsson, J. Tandem Cladding-Pumping for Control of Excess Gain in Ytterbium-Doped Fiber Amplifiers. IEEE J. Quantum Electron. 2010, 46, 1860-1869, doi:10.1109/JQE.2010.2076408.

87. Dai, J.; Li, F.; Liu, N.; Shen, C.; Zhang, L.; Li, H.; Li, Y.; Sun, S.; Li, Y.; Lv, J.; et al. Extraction of more than $10 \mathrm{~kW}$ from Ybdoped tandem-pumping aluminophosphosilicate fiber. In Proceedings of the Global Intelligent Industry Conference 2020; Wang, L., Ed.; SPIE, 2021; Vol. 11780, p. 76.

88. Zhou, P.; Xiao, H.; Leng, J.; Xu, J.; Chen, Z.; Zhang, H.; Liu, Z. High-power fiber lasers based on tandem pumping. J. Opt. Soc. 
Am. B 2017, 34, A29, doi:10.1364/JOSAB.34.000A29.

89. Glick, Y.; Sintov, Y.; Zuitlin, R.; Pearl, S.; Shamir, Y.; Feldman, R.; Horvitz, Z.; Shafir, N. Single-mode 230 W output power $1018 \mathrm{~nm}$ fiber laser and ASE competition suppression. J. Opt. Soc. Am. B 2016, 33, 1392, doi:10.1364/JOSAB.33.001392.

90. Khitrov, V.; Samson, B.; Machewirth, D.; Tankala, K. 242W single -mode CW fiber laser operating at 1030nm lasing wavelength and with $0.35 \mathrm{~nm}$ spectral width. In Proceedings of the Advanced Solid-State Photonics; OSA: Washington, D.C., 2006; p. WD5.

91. Ottenhues, C.; Theeg, T.; Hausmann, K.; Wysmolek, M.; Sayinc, H.; Neumann, J.; Kracht, D. Single-mode monolithic fiber laser with $200 \mathrm{~W}$ output power at a wavelength of $1018 \mathrm{~nm}$. Opt. Lett. 2015, 40, 4851, doi:10.1364/OL.40.004851.

92. Wang, X.; Yan, P.; Wang, Z.; Huang, Y.; Tian, J.; Li, D.; Xiao, Q. The $5.4 \mathrm{~kW}$ output power of the ytterbium-doped tandempumping fiber amplifier. 2018 Conf. Lasers Electro-Optics, CLEO 2018 - Proc. 2018, 4-5.

93. Lafouti, M.; Latifi, H.; Sarabi, H.; Fathi, H.; Ebrahimzadeh, S.; Sarikhani, S. 407 W specially-designed fiber laser at 1018 nm using a gain fiber with a low core/cladding ratio of 20/400 $\mu$ m. Laser Phys. 2018, 28, 115102, doi:10.1088/1555-6611/aad92f.

94. Lafouti, M.; Latifi, H.; Fathi, H.; Ebrahimzadeh, S.; Sarikhani, S.; Sarabi, H. Experimental investigation of a high-power 1018 nm fiber laser using a 20/400 $\mu \mathrm{m}$ ytterbium-doped fiber. Appl. Opt. 2019, 58, 729, doi:10.1364/AO.58.000729.

95. Yan, P.; Wang, X.; Wang, Z.; Huang, Y.; Li, D.; Xiao, Q.; Gong, M. A 1150-W 1018-nm Fiber Laser Bidirectional Pumped by Wavelength-Stabilized Laser Diodes. IEEE J. Sel. Top. Quantum Electron. 2018, 24, 1-6, doi:10.1109/JSTQE.2018.2805801.

96. V. Fomin, M. Abramov, A. Ferin, A. Abramov, D. Mochalov, N. Platonov, and V.G. 10 kW single-mode fiber laser. In Proceedings of the 5th International Symposium on High-Power Fiber Lasers and Their Applications; 2010.

97. Ferin A, Gapontsev V, Fomin V, Abramov A, A.M. and M.D. $17 \mathrm{~kW} \mathrm{CW} \mathrm{laser} \mathrm{with} 50 \mu \mathrm{m}$ delivery fiber. 15th Int. Conf. Laser Opt. (St Petersburg, June 2012). 12AD.

98. Yao, T.; Ji, J.; Nilsson, J. Ultra-Low Quantum-Defect Heating in Ytterbium-Doped Aluminosilicate Fibers. J. Light. Technol. 2014, 32, 429-434, doi:10.1109/JLT.2013.2290284.

99. Naderi, S.; Dajani, I.; Grosek, J.; Madden, T.; Dinh, T.-N. Theoretical analysis of effect of pump and signal wavelengths on modal instabilities in Yb-doped fiber amplifiers. https://doi.org/10.1117/12.2044143 2014, 8964, 264-270, doi:10.1117/12.2044143.

100. Fu, S.; Feng, X.; Si, L.; Guo, Z.; Jia, X.; Zhao, Y.; Yuan, S.; Dong, X. Self-pulsing dynamics of high-power Yb-doped fiber lasers. Microw. Opt. Technol. Lett. 2006, 48, 2282-2285, doi:10.1002/mop.21914.

101. Limpert, J.; Klenke, A.; Kienel, M.; Breitkopf, S.; Eidam, T.; Hadrich, S.; Jauregui, C.; Tunnermann, A. Performance Scaling of Ultrafast Laser Systems by Coherent Addition of Femtosecond Pulses. IEEE J. Sel. Top. Quantum Electron. 2014, 20, 268-277, doi:10.1109/JSTQE.2014.2304135.

102. Klenke, A.; Muller, M.; Stark, H.; Kienel, M.; Jauregui, C.; Tunnermann, A.; Limpert, J. Coherent Beam Combination of Ultrafast Fiber Lasers. IEEE J. Sel. Top. Quantum Electron. 2018, 24, 1-9, doi:10.1109/JSTQE.2018.2808540.

103. Klenke, A.; Seise, E.; Limpert, J.; Tünnermann, A. Basic considerations on coherent combining of ultrashort laser pulses. Opt. Express 2011, 19, 25379, doi:10.1364/OE.19.025379.

104. Uberna, R.; Bratcher, A.; Tiemann, B.G. Coherent Polarization Beam Combination. IEEE J. Quantum Electron. 2010, 46, 11911196, doi:10.1109/JQE.2010.2044976.

105. Fan, T.Y. Laser beam combining for high-power, high-radiance sources. IEEE J. Sel. Top. Quantum Electron. 2005, 11, 567-577, doi:10.1109/JSTQE.2005.850241.

106. Augst, S.J.; Ranka, J.K.; Fan, T.Y.; Sanchez, A. Beam combining of ytterbium fiber amplifiers (Invited). J. Opt. Soc. Am. B 2007, 24, 1707, doi:10.1364/JOSAB.24.001707.

107. Liu, Z.; Zhou, P.; Xu, X.; Wang, X.; Ma, Y. Coherent beam combining of high power fiber lasers: Progress and prospect. Sci. China Technol. Sci. 2013, 56, 1597-1606, doi:10.1007/s11431-013-5260-z.

108. Liu, Z.; Ma, P.; Su, R.; Tao, R.; Ma, Y.; Wang, X.; Zhou, P. High-power coherent beam polarization combination of fiber lasers: 
progress and prospect [Invited]. J. Opt. Soc. Am. B 2017, 34, A7, doi:10.1364/JOSAB.34.0000A7.

109. Bourdon, P.; Le Gouet, J.; Goular, D.; Lombard, L.; Durecu, A. Coherent combining with active phase control: A practical tool for adaptive and nonlinear optics. In Proceedings of the 2017 Conference on Lasers and Electro-Optics Pacific Rim (CLEOPR); IEEE, 2017; Vol. Part F122-, pp. 1-3.

110. Hanna, M.; Guichard, F.; Zaouter, Y.; Papadopoulos, D.N.; Druon, F.; Georges, P. Coherent combination of ultrafast fiber amplifiers. J. Phys. B At. Mol. Opt. Phys. 2016, 49, 062004, doi:10.1088/0953-4075/49/6/062004.

111. Sprangle, P.; Ting, A.; Penano, J.; Fischer, R.; Hafizi, B. Incoherent Combining and Atmospheric Propagation of High-Power Fiber Lasers for Directed-Energy Applications. IEEE J. Quantum Electron. 2009, 45, 138-148, doi:10.1109/JQE.2008.2002501.

112. Sprangle, P.; Hafizi, B.; Ting, A.; Fischer, R. High-power lasers for directed-energy applications. Appl. Opt. 2015, 54, F201, doi:10.1364/AO.54.00F201.

113. Lockheed Martin demonstrates $30 \mathrm{~kW}$ weapons-grade fiber laser Available online: https://www.laserfocusworld.com/laserssources/article/16564243/lockheed-martin-demonstrates-30-kw-weaponsgrade-fiber-laser.

114. Zhu, Y.; Li, P.; Li, C.; Wang, L.; Yao, C.; Zhang, X.; Li, S.; Zhou, Y. Quantizing the coherent polarization beam combination from temporal, spatial, and spectral domains. In Proceedings of the Fourth International Symposium on High Power Laser Science and Engineering (HPLSE 2021); Zhu, J., Ed.; SPIE, 2021; Vol. 11849, p. 18.

115. Lei, C.; Gu, Y.; Chen, Z.; Wang, Z.; Zhou, P.; Ma, Y.; Xiao, H.; Leng, J.; Wang, X.; Hou, J.; et al. Incoherent beam combining of fiber lasers by an all-fiber $7 \times 1$ signal combiner at a power level of $14 \mathrm{~kW}$. Opt. Express 2018, 26, 10421, doi:10.1364/OE.26.010421.

116. Goodno, G.D.; Asman, C.P.; Anderegg, J.; Brosnan, S.; Cheung, E.C.; Hammons, D.; Injeyan, H.; Komine, H.; Long, W.H.; McClellan, M.; et al. Brightness-Scaling Potential of Actively Phase-Locked Solid-State Laser Arrays. IEEE J. Sel. Top. Quantum Electron. 2007, 13, 460-472, doi:10.1109/JSTQE.2007.896618.

117. Augst, S.J.; Goyal, A.K.; Aggarwal, R.L.; Fan, T.Y.; Sanchez, A. Wavelength beam combining of ytterbium fiber lasers. Opt. Lett. 2003, 28, 331, doi:10.1364/OL.28.000331.

118. Sevian, A.; Andrusyak, O.; Ciapurin, I.; Smirnov, V.; Venus, G.; Glebov, L. Efficient power scaling of laser radiation by spectral-beam combining: erratum. Opt. Lett. 2008, 33, 760, doi:10.1364/OL.33.000760.

119. Zhu, Z.; Gou, L.; Jiang, M.; Hui, Y.; Lei, H.; Li, Q. High beam quality in two directions and high efficiency output of a diode laser array by spectral-beam-combining. Opt. Express 2014, 22, 17804, doi:10.1364/OE.22.017804.

120. Antonio Sanchez-Rubio, Tso Yee Fan, Steven J. Augst, Anish K. Goyal, K.J.; Creedon, Juliet T. Gopinath, Vincenzo Daneu, Bien Chann, and R.H. Wavelength Beam Combining for Power and Brightness Scaling of Laser Systems. MIT-Lincoln Lab. J. 2014, 20, 52-66.

121. Wirth, C.; Schmidt, O.; Tsybin, I.; Schreiber, T.; Eberhardt, R.; Limpert, J.; Tünnermann, A.; Ludewigt, K.; Gowin, M.; ten Have, E.; et al. High average power spectral beam combining of four fiber amplifiers to $82 \mathrm{~kW}$. Opt. Lett. 2011, 36, 3118, doi:10.1364/OL.36.003118.

122. Schmidt, O.; Rekas, M.; Wirth, C.; Rothhardt, J.; Rhein, S.; Kliner, A.; Strecker, M.; Schreiber, T.; Limpert, J.; Eberhardt, R.; et al. High power narrow-band fiber-based ASE source. Opt. Express 2011, 19, 4421, doi:10.1364/OE.19.004421.

123. Xu, J.; Liu, W.; Leng, J.; Xiao, H.; Guo, S.; Zhou, P.; Chen, J. Power scaling of narrowband high-power all-fiber superfluorescent fiber source to $1.87 \mathrm{~kW}$. Opt. Lett. 2015, 40, 2973, doi:10.1364/OL.40.002973.

124. Zheng, Y.; Yang, Y.; Wang, J.; Hu, M.; Liu, G.; Zhao, X.; Chen, X.; Liu, K.; Zhao, C.; He, B.; et al. 10.8 kW spectral beam combination of eight all-fiber superfluorescent sources and their dispersion compensation. Opt. Express 2016, 24, 12063, doi:10.1364/OE.24.012063.

125. Zheng, Y.; Zhu, Z.; Liu, X.; Yu, M.; Li, S.; Zhang, L.; Ni, Q.; Wang, J.; Wang, X. High-power, high-beam-quality spectral beam combination of six narrow-linewidth fiber amplifiers with two transmission diffraction gratings. Appl. Opt. 2019, 58, 8339, 


\section{doi:10.1364/AO.58.008339.}

126. Chen, F.; Ma, J.; Wei, C.; Zhu, R.; Zhou, W.; Yuan, Q.; Pan, S.; Zhang, J.; Wen, Y.; Dou, J. 10 kW-level spectral beam combination of two high power broad-linewidth fiber lasers by means of edge filters. Opt. Express 2017, 25, 32783, doi:10.1364/OE.25.032783.

127. Wirth, C.; Schmidt, O.; Tsybin, I.; Schreiber, T.; Peschel, T.; Brückner, F.; Clausnitzer, T.; Limpert, J.; Eberhardt, R.; Tünnermann, A.; et al. $2 \mathrm{~kW}$ incoherent beam combining of four narrow-linewidth photonic crystal fiber amplifiers. Opt. Express 2009, 17, 1178, doi:10.1364/OE.17.001178.

128. Madasamy, P.; Loftus, T.; Thomas, A.; Jones, P.; Honea, E. Comparison of spectral beam combining approaches for high power fiber laser systems. In Proceedings of the Laser Source Technology for Defense and Security IV; Dubinskii, M., Wood, G.L., Eds.; 2008; Vol. 6952, p. 695207.

129. Shi, C.; Zhang, H.; Wang, X.; Zhou, P.; Xu, X. kW-class high power fiber laser enabled by active long tapered fiber. High Power Laser Sci. Eng. 2018, 6, e16, doi:10.1017/hpl.2018.9.

130. Ma, J.; Chen, F.; Wei, C.; Zhu, R. Modeling and Analysis of the Influence of an Edge Filter on the Combining Efficiency and Beam Quality of a 10-kW-Class Spectral Beam-Combining System. Appl. Sci. 2019, 9, 2152, doi:10.3390/app9102152.

131. Lockheed Martin to Deliver World Record-Setting 60kW Laser to U.S. Army - Mar 16, 2017 Available online: https://news.lockheedmartin.com/2017-03-16-Lockheed-Martin-to-Deliver-World-Record-Setting-60kW-Laser-to-U-S-Army (accessed on May 28, 2021).

132. Honea, E.; Afzal, R.S.; Savage-Leuchs, M.; Henrie, J.; Brar, K.; Kurz, N.; Jander, D.; Gitkind, N.; Hu, D.; Robin, C.; et al. Advances in fiber laser spectral beam combining for power scaling. In Proceedings of the Components and Packaging for Laser Systems II; Glebov, A.L., Leisher, P.O., Eds.; SPIE, 2016; Vol. 9730, p. 97300Y.

133. Lockheed Martin to Deliver World Record-Setting 60kW Laser to U.S. Army - Mar 16, 2017 Available online: https://news.lockheedmartin.com/2017-03-16-Lockheed-Martin-to-Deliver-World-Record-Setting-60kW-Laser-to-U-S-Army (accessed on Sep 8, 2021).

134. Brignon, A. Coherent laser beam combining. 2013, 481.

135. Ripper, J.E.; Paoli, T.L. OPTICAL COUPLING OF ADJACENT STRIPE-GEOMETRY JUNCTION LASERS. Appl. Phys. Lett. 1970, 17, 371-373, doi:10.1063/1.1653440.

136. Kozlov, V.A.; Hernández-Cordero, J.; Morse, T.F. All-fiber coherent beam combining of fiber lasers. Opt. Lett. 1999, 24, 1814, doi:10.1364/OL.24.001814.

137. Cheung, E.C.; Weber, M.; Rice, R.R. Phase Locking of a Pulsed Fiber Amplifier. In Proceedings of the Advanced Solid-State Photonics; OSA: Washington, D.C., 2008; p. WA2.

138. McNaught, S.J.; Asman, C.P.; Injeyan, H.; Jankevics, A.; Johnson, A.M.F.; Jones, G.C.; Komine, H.; Machan, J.; Marmo, J.; McClellan, M.; et al. 100-kW Coherently Combined Nd:YAG MOPA Laser Array. In Proceedings of the Frontiers in Optics 2009/Laser Science XXV/Fall 2009 OSA Optics \& Photonics Technical Digest; OSA: Washington, D.C., 2009 ; p. FThD2.

139. Goodno, G.D.; Shih, C.; Rothenberg, J.E. Perturbative analysis of coherent combining efficiency with mismatched lasers. Opt. Express 2010, 18, 25403, doi:10.1364/OE.18.025403.

140. Leshchenko, V.E. Coherent combining efficiency in tiled and filled aperture approaches. Opt. Express 2015, 23, 15944, doi:10.1364/OE.23.015944.

141. Prossotowicz, M.; Heimes, A.; Flamm, D.; Jansen, F.; Otto, H.-J.; Budnicki, A.; Killi, A.; Morgner, U. Coherent beam combining with micro-lens arrays. Opt. Lett. 2020, 45, 6728, doi:10.1364/OL.414388.

142. Zhi, D.; Zhang, Z.; Ma, Y.; Wang, X.; Chen, Z.; Wu, W.; Zhou, P.; Si, L. Realization of large energy proportion in the central lobe by coherent beam combination based on conformal projection system. Sci. Rep. 2017, 7, 2199, doi:10.1038/s41598-01702118-z. 
143. Scifres, D.R.; Streifer, W.; Burnham, R.D. High-power coupled-multiple-stripe phase-locked injection laser. Appl. Phys. Lett. 1979, 34, 259-261, doi:10.1063/1.90773.

144. Tan, Y.; Li, X. Impact of filling factor on correction of piston and tip/tilt in coherent beam combination. In Proceedings of the 14th National Conference on Laser Technology and Optoelectronics (LTO 2019); Xu, H.-L., Chen, F., Ji, L., Li, B., Xie, X., Leng, Y., Sheng, Z., Yang, Y., Zhao, J., Zhu, J., Zhang, Z., Zhong, M., Qiu, J., Hu, M., Qiu, J., Hu, W., Dai, S., Cai, Y., Shi, K., Yao, J., Zhang, Z., Xu, S., Zhao, D., Shan, C., Ding, Y., Zheng, Y., Chen, W., Wang, P., Eds.; SPIE, 2019; Vol. 11170, p. 215.

145. Lachinova, S.L.; Vorontsov, M.A. Laser beam projection with adaptive array of fiber collimators II Analysis of atmospheric compensation efficiency. J. Opt. Soc. Am. A 2008, 25, 1960, doi:10.1364/josaa.25.001960.

146. Le Dortz, J.; Heilmann, A.; Antier, M.; Bourderionnet, J.; Larat, C.; Fsaifes, I.; Daniault, L.; Bellanger, S.; Simon Boisson, C.; Chanteloup, J.-C.; et al. Highly scalable femtosecond coherent beam combining demonstrated with 19 fibers. Opt. Lett. 2017, 42, 1887, doi:10.1364/OL.42.001887.

147. Yu, C.X.; Augst, S.J.; Redmond, S.M.; Goldizen, K.C.; Murphy, D. V.; Sanchez, A.; Fan, T.Y. Coherent combining of a 4 kW, eight-element fiber amplifier array. Opt. Lett. 2011, 36, 2686, doi:10.1364/OL.36.002686.

148. Bourderionnet, J.; Bellanger, C.; Primot, J.; Brignon, A. Coherent phase combining of 64 fibers. In Proceedings of the 2011 Conference on Lasers and Electro-Optics Europe and 12th European Quantum Electronics Conference (CLEO EUROPE/EQEC); IEEE, 2011; Vol. 19, pp. 1-1.

149. Weyrauch, T.; Vorontsov, M.; Mangano, J.; Ovchinnikov, V.; Bricker, D.; Polnau, E.; Rostov, A. Deep turbulence effects mitigation with coherent combining of 21 laser beams over 7 km. Opt. Lett. 2016, 41, 840, doi:10.1364/OL.41.000840.

150. Huang, Z.; Tang, X.; Luo, Y.; Liu, C.; Li, J.; Zhang, D.; Wang, X.; Chen, T.; Han, M. Active phase locking of thirty fiber channels using multilevel phase dithering method. Rev. Sci. Instrum. 2016, 87, 033109, doi:10.1063/1.4943666.

151. Kabeya, D.; Kermène, V.; Fabert, M.; Benoist, J.; Saucourt, J.; Desfarges-Berthelemot, A.; Barthélémy, A. Efficient phaselocking of 37 fiber amplifiers by phase-intensity mapping in an optimization loop. Opt. Express 2017, 25, 13816, doi:10.1364/OE.25.013816.

152. Fsaifes, I.; Daniault, L.; Bellanger, S.; Veinhard, M.; Bourderionnet, J.; Larat, C.; Lallier, E.; Durand, E.; Brignon, A.; Chanteloup, J.-C. Coherent beam combining of 61 femtosecond fiber amplifiers. Opt. Express 2020, 28, 20152, doi:10.1364/OE.394031.

153. Du, Q.; Wang, D.; Zhou, T.; Li, D.; Wilcox, R. Characterization and Control of 81-beam Diffractive Coherent Combining. In Proceedings of the Laser Congress 2020 (ASSL, LAC); OSA: Washington, D.C., 2020; p. ATu4A.5.

154. Chang, H.; Chang, Q.; Xi, J.; Hou, T.; Su, R.; Ma, P.; Wu, J.; Li, C.; Jiang, M.; Ma, Y.; et al. First experimental demonstration of coherent beam combining of more than 100 beams. Photonics Res. 2020, 8, 1943, doi:10.1364/PRJ.409788.

155. Klenke, A.; Müller, M.; Stark, H.; Stutzki, F.; Hupel, C.; Schreiber, T.; Tünnermann, A.; Limpert, J. Coherently combined 16channel multicore fiber laser system. Opt. Lett. 2018, 43, 1519, doi:10.1364/OL.43.001519.

156. Ramirez, L.P.; Hanna, M.; Bouwmans, G.; El Hamzaoui, H.; Bouazaoui, M.; Labat, D.; Delplace, K.; Pouysegur, J.; Guichard, F.; Rigaud, P.; et al. Coherent beam combining with an ultrafast multicore Yb-doped fiber amplifier. Opt. Express 2015, 23, 5406, doi:10.1364/OE.23.005406.

157. Elkin, N.N.; Napartovich, A.P.; Sukharev, A.G.; Troschieva, V.N.; Vysotsky, D.V. Direct numerical simulation of radiation propagation in a multicore fiber. Opt. Commun. 2000, 177, 207-217, doi:10.1016/S0030-4018(00)00556-3.

158. Wrage, M.; Glas, P.; Fischer, D.; Leitner, M.; Vysotsky, D. V.; Napartovich, A.P. Phase locking in a multicore fiber laser by means of a Talbot resonator. Opt. Lett. 2000, 25, 1436, doi:10.1364/OL.25.001436.

159. Gorton, E.K.; Jenkins, R.M. Theory of 1-N-way phase-locked resonators. Appl. Opt. 2001, 40, 916, doi:10.1364/AO.40.000916.

160. Vysotsky, D. V; Napartovich, A.P. Coherent beam combining in optically coupled laser arrays. Quantum Electron. 2019, 49, 989-1007, doi:10.1070/QEL16978. 
161. Michaille, L.; Bennett, C.R.; Taylor, D.M.; Shepherd, T.J.; Broeng, J.; Simonsen, H.R.; Petersson, A. Phase locking and supermode selection in multicore photonic crystal fiber lasers with a large doped area. Opt. Lett. 2005, 30, 1668, doi:10.1364/OL.30.001668.

162. Fang, X.-H.; Hu, M.-L.; Liu, B.-W.; Chai, L.; Wang, C.-Y.; Zheltikov, A.M. Generation of 150 MW, 110 fs pulses by phaselocked amplification in multicore photonic crystal fiber. Opt. Lett. 2010, 35, 2326, doi:10.1364/OL.35.002326.

163. Paurisse, M.; Hanna, M.; Druon, F.; Georges, P. Wavefront control by digital holography in an Yb-doped multi-core fiber amplifier. In Proceedings of the Conference on Lasers and Electro-Optics 2010; OSA: Washington, D.C., 2010; Vol. 35, p. CThO4.

164. Steinkopff, A.; Jauregui, C.; Aleshire, C.; Klenke, A.; Limpert, J. Optimizing the design of coherently combined multicore fiber amplifiers. In Proceedings of the Fiber Lasers XVIII: Technology and Systems; Zervas, M.N., Jauregui-Misas, C., Eds.; SPIE, 2021; Vol. 11665, p. 58.

165. Lin, D.; Carpenter, J.; Feng, Y.; Jain, S.; Jung, Y.; Feng, Y.; Zervas, M.N.; Richardson, D.J. Reconfigurable structured light generation in a multicore fibre amplifier. Nat. Commun. 2020, 11, 3986, doi:10.1038/s41467-020-17809-x.

166. Weyrauch, T.; Vorontsov, M.; Mangano, J.; Ovchinnikov, V.; Bricker, D.; Polnau, E.; Rostov, A. Deep turbulence effects mitigation with coherent combining of 21 laser beams over 7 km. Opt. Lett. 2016, 41, 840, doi:10.1364/OL.41.000840.

167. Shekel, E.; Vidne, Y.; Urbach, B. 16kW single mode CW laser with dynamic beam for material processing. In Proceedings of the Fiber Lasers XVII: Technology and Systems; Dong, L., Zervas, M.N., Eds.; SPIE, 2020; Vol. 11260, p. 73.

168. Ma, P.; Chang, H.; Ma, Y.; Su, R.; Qi, Y.; Wu, J.; Li, C.; Long, J.; Lai, W.; Chang, Q.; et al. 7.1 kW coherent beam combining system based on a seven-channel fiber amplifier array. Opt. Laser Technol. 2021, 140, 107016, doi:10.1016/j.optlastec.2021.107016.

169. Müller, M.; Aleshire, C.; Klenke, A.; Haddad, E.; Légaré, F.; Tünnermann, A.; Limpert, J. 10.4 kW coherently combined ultrafast fiber laser. Opt. Lett. 2020, 45, 3083, doi:10.1364/OL.392843.

170. Regelskis, K.; Gavrilinas, N.; Trusovas, R.; Račiukaitis, G. Coherent addition of orthogonally polarized fibre lasers with high combining efficiency. Lith. J. Phys. 2010, 50, 209-214, doi:10.3952/lithjphys.50208.

171. Seise, E.; Klenke, A.; Limpert, J.; Tünnermann, A. Coherent addition of fiber-amplified ultrashort laser pulses. Opt. Express 2010, 18, 27827, doi:10.1364/OE.18.027827.

172. Klenke, A.; Breitkopf, S.; Kienel, M.; Gottschall, T.; Eidam, T.; Hädrich, S.; Rothhardt, J.; Limpert, J.; Tünnermann, A. 530 W, $13 \mathrm{~mJ}$, four-channel coherently combined femtosecond fiber chirped-pulse amplification system. Opt. Lett. 2013, 38, 2283, doi:10.1364/OL.38.002283.

173. Kienel, M.; Müller, M.; Klenke, A.; Limpert, J.; Tünnermann, A. $12 \mathrm{~mJ}$ kW-class ultrafast fiber laser system using multidimensional coherent pulse addition. Opt. Lett. 2016, 41, 3343, doi:10.1364/OL.41.003343.

174. Müller, M.; Kienel, M.; Klenke, A.; Gottschall, T.; Shestaev, E.; Plötner, M.; Limpert, J.; Tünnermann, A. 1 kW 1 mJ eightchannel ultrafast fiber laser. Opt. Lett. 2016, 41, 3439, doi:10.1364/OL.41.003439.

175. Stark, H.; Buldt, J.; Müller, M.; Klenke, A.; Tünnermann, A.; Limpert, J. 23 mJ high-power fiber CPA system using electrooptically controlled divided-pulse amplification. Opt. Lett. 2019, 44, 5529, doi:10.1364/OL.44.005529.

176. Stark, H.; Buldt, J.; Müller, M.; Klenke, A.; Limpert, J. 1 kW, 10 mJ, 120 fs coherently combined fiber CPA laser system. Opt. Lett. 2021, 46, 969, doi:10.1364/OL.417032.

177. Müller, M.; Aleshire, C.; Buldt, J.; Stark, H.; Grebing, C.; Klenke, A.; Limpert, J. Scaling potential of beam-splitter-based coherent beam combination. Opt. Express 2021, 29, 27900, doi:10.1364/OE.433596.

178. Veldkamp, W.B.; Leger, J.R.; Swanson, G.J. Coherent summation of laser beams using binary phase gratings. Opt. Lett. 1986, 11, 303, doi:10.1364/OL.11.000303.

179. Ehbets, P.; Gale, M.T.; Herzig, H.P.; Prongué, D. High-efficiency continuous surface-relief gratings for two-dimensional array 
generation. Opt. Lett. 1992, 17, 908, doi:10.1364/OL.17.000908.

180. Coherent Laser Beam Combining - Google Books Available online: https://books.google.fi/books/about/Coherent_Laser_Beam_Combining.html?id=OccZAgAAQBAJ\&source=kp_book_descri ption\&redir_esc=y (accessed on Jun 8, 2021).

181. Flores, A.; Dajani, I.; Holten, R.; Ehrenreich, T.; Anderson, B. Multi-kilowatt diffractive coherent combining of pseudorandom-modulated fiber amplifiers. Opt. Eng. 2016, 55, 096101, doi:10.1117/1.OE.55.9.096101.

182. Hebling, J. Derivation of the pulse front tilt caused by angular dispersion. Opt. Quantum Electron. 1996, 28, 1759-1763, doi:10.1007/BF00698541.

183. Zhou, T.; Sano, T.; Wilcox, R. Coherent combination of ultrashort pulse beams using two diffractive optics. Opt. Lett. 2017, 42, 4422, doi:10.1364/OL.42.004422.

184. Cheung, E.C.; Ho, J.G.; Goodno, G.D.; Rice, R.R.; Rothenberg, J.; Thielen, P.; Weber, M.; Wickham, M. Diffractive-opticsbased beam combination of a phase-locked fiber laser array. Opt. Lett. 2008, 33, 354, doi:10.1364/OL.33.000354.

185. Redmond, S.M.; Ripin, D.J.; Yu, C.X.; Augst, S.J.; Fan, T.Y.; Thielen, P.A.; Rothenberg, J.E.; Goodno, G.D. Diffractive coherent combining of a $25 \mathrm{~kW}$ fiber laser array into a $19 \mathrm{~kW}$ Gaussian beam. Opt. Lett. 2012, 37, 2832, doi:10.1364/OL.37.002832.

186. Thielen, P.A.; Ho, J.G.; Burchman, D.A.; Goodno, G.D.; Rothenberg, J.E.; Wickham, M.G.; Flores, A.; Lu, C.A.; Pulford, B.; Robin, C.; et al. Two-dimensional diffractive coherent combining of 15 fiber amplifiers into a $600 \mathrm{~W}$ beam. Opt. Lett. 2012, 37, 3741, doi:10.1364/OL.37.003741.

187. McNaught, S.J.; Thielen, P.A.; Adams, L.N.; Ho, J.G.; Johnson, A.M.; Machan, J.P.; Rothenberg, J.E.; Chun-Ching Shih; Shimabukuro, D.M.; Wacks, M.P.; et al. Scalable Coherent Combining of Kilowatt Fiber Amplifiers Into a 2.4-kW Beam. IEEE J. Sel. Top. Quantum Electron. 2014, 20, 174-181, doi:10.1109/JSTQE.2013.2296771.

188. Wilcox, R.; Dahlen, D.; Sano, T. Femtosecond Beam Combination Using Diffractive Optic Pairs. In Proceedings of the Conference on Lasers and Electro-Optics; OSA: Washington, D.C., 2017; Vol. 2017-Janua, p. SM4I.2.

189. Zhou, T.; Du, Q.; Sano, T.; Wilcox, R.; Leemans, W. Two-dimensional combination of eight ultrashort pulsed beams using a diffractive optic pair. Opt. Lett. 2018, 43, 3269, doi:10.1364/OL.43.003269.

190. Du, Q.; Zhou, T.; Doolittle, L.R.; Huang, G.; Li, D.; Wilcox, R. Deterministic stabilization of eight-way 2D diffractive beam combining using pattern recognition. Opt. Lett. 2019, 44, 4554, doi:10.1364/OL.44.004554.

191. Liu, M.; Shen, H.; Yang, Y.; Xian, Y.; Zhang, J.; Wang, H.; Li, B.; Niu, X.; He, B. Investigation of combining-efficiency loss induced by a diffractive optical element in a single-aperture coherent beam combining system. Opt. Express 2021, 29, 5179, doi:10.1364/OE.413459.

192. Klenke, A.; Müller, M.; Stark, H.; Tünnermann, A.; Limpert, J. Sequential phase locking scheme for a filled aperture intensity coherent combination of beam arrays. Opt. Express 2018, 26, 12072, doi:10.1364/OE.26.012072.

193. Aleshire, C.; Steinkopff, A.; Jauregui, C.; Klenke, A.; Tünnermann, A.; Limpert, J. Simplified design of optical elements for filled-aperture coherent beam combination. Opt. Express 2020, 28, 21035, doi:10.1364/OE.394084.

194. Harder, I.; Lano, M.; Lindlein, N.; Schwider, J. Homogenization and beam shaping with microlens arrays. In Proceedings of the Photon Management; SPIE, 2004; Vol. 5456, p. 99.

195. Zimmermann, M.; Lindlein, N.; Voelkel, R.; Weible, K.J. Microlens laser beam homogenizer: from theory to application. Laser Beam Shap. VIII 2007, 6663, 666302, doi:10.1117/12.731391.

196. Jin, Y.; Hassan, A.; Jiang, Y. Freeform microlens array homogenizer for excimer laser beam shaping. Opt. Express 2016, 24, 24846, doi:10.1364/OE.24.024846.

197. Streibl, N.; Nölscher, U.; Jahns, J.; Walker, S. Array generation with lenslet arrays. Appl. Opt. 1991, 30, 2739, doi:10.1364/AO.30.002739.

198. Prossotowicz, M.; Heimes, A.; Flamm, D.; Jansen, F.; Otto, H.-J.; Budnicki, A.; Morgner, U.; Killi, A. Dynamic coherent beam 
combining based on a setup of microlens arrays. In Proceedings of the Laser Resonators, Microresonators, and Beam Control XXII; Armani, A.M., Kudryashov, A. V., Paxton, A.H., Ilchenko, V.S., Eds.; SPIE, 2020; Vol. 1126612, p. 38.

199. Prossotowicz, M.; Flamm, D.; Heimes, A.; Jansen, F.; Otto, H.-J.; Budnicki, A.; Killi, A.; Morgner, U. Dynamic focus shaping with mixed-aperture coherent beam combining. Opt. Lett. 2021, 46, 1660, doi:10.1364/OL.422135.

200. Kringlebotn, J.T.; Archambault, J.-L.; Reekie, L.; Payne, D.N. Er3+:Yb3+-codoped fiber distributed-feedback laser. Opt. Lett. 1994, 19, 2101, doi:10.1364/OL.19.002101.

201. Yelen, K.; Zervas, M.N.; Hickey, L.M.B. Fibre DFB lasers with ultimate efficiency. OSA Trends Opt. Photonics Ser. 2004, 95 B, 703-705.

202. Bjarklev, A.; Broeng, J.; Bjarklev, A.S. Photonic Crystal Fibres; Springer US: Boston, MA, 2003; ISBN 978-1-4613-5095-8.

203. Limpert, J.; Liem, A.; Reich, M.; Schreiber, T.; Nolte, S.; Zellmer, H.; Tunnermann, A.; Broeng, J.; Petersson, A.; Jakobsen, C. Low-nonlinearity single-transverse-mode ytterbium-doped photonic crystal fiber amplifier. Opt. Express 2004, 12, 1313, doi:10.1364/OPEX.12.001313.

204. Kawamura, T.; Shirakawa, A.; Saito, K. Phase-locked and mode-locked multicore photonic crystal fiber laser with a saturable absorber. Opt. Express 2021, 29, 17023, doi:10.1364/OE.421083.

205. Limpert, J.; Stutzki, F.; Jansen, F.; Otto, H.-J.; Eidam, T.; Jauregui, C.; Tünnermann, A. Yb-doped large-pitch fibres: effective single-mode operation based on higher-order mode delocalisation. Light Sci. Appl. 2012, 1, e8-e8, doi:10.1038/lsa.2012.8.

206. Filippov, V.; Chamorovskii, Y.; Kerttula, J.; Golant, K.; Pessa, M.; Okhotnikov, O.G. Double clad tapered fiber for high power applications. Opt. Express 2008, 16, 1929, doi:10.1364/OE.16.001929.

207. Filippov, V.; Chamorovskii, Y.K.; Golant, K.M.; Vorotynskii, A.; Okhotnikov, O.G. Optical amplifiers and lasers based on tapered fiber geometry for power and energy scaling with low signal distortion. In Proceedings of the Fiber Lasers XIII: Technology, Systems, and Applications; Ballato, J., Ed.; 2016; Vol. 9728, p. 97280V.

208. Kerttula, J.; Filippov, V.; Chamorovskii, Y.; Ustimchik, V.; Golant, K.; Okhotnikov, O.G. Principles and performance of tapered fiber lasers: from uniform to flared geometry. Appl. Opt. 2012, 51, 7025, doi:10.1364/AO.51.007025.

209. Huang, L.; Ma, P.; Su, R.; Lai, W.; Ma, Y.; Zhou, P. Comprehensive investigation on the power scaling of a tapered Yb-doped fiber-based monolithic linearly polarized high-peak-power near-transform-limited nanosecond fiber laser. Opt. Express 2021, 29, 761, doi:10.1364/OE.414788.

210. Bobkov, K.K.; Aleshkina, S.S.; Khudyakov, M.M.; Lipatov, D.S.; Likhachev, M.E. Active tapered fibers for high peak power fiber lasers. In Proceedings of the Micro-structured and Specialty Optical Fibres VII; Peterka, P., Kalli, K., Mendez, A., Eds.; SPIE, 2021; Vol. 11773, p. 7.

211. Guesmi, K.; Mugnier, A.; Canat, G.; Canal, C.; Maine, P. Simple design for high energy femtosecond tapered double clad fiber amplifier. In Proceedings of the Fiber Lasers XVIII: Technology and Systems; Zervas, M.N., Jauregui-Misas, C., Eds.; SPIE, 2021; Vol. 11665, p. 39.

212. Ye, Y.; Xi, X.; Shi, C.; Yang, B.; Wang, X.; Zhang, H.; Zhou, P.; Xu, X. Comparative study on transverse mode instability of fiber amplifiers based on long tapered fiber and conventional uniform fiber. Laser Phys. Lett. 2019, 16, 085109, doi:10.1088/1612-202X/ab2acf.

213. Fedotov, A.; Noronen, T.; Gumenyuk, R.; Ustimchik, V.; Chamorovskii, Y.; Golant, K.; Odnoblyudov, M.; Rissanen, J.; Niemi, T.; Filippov, V. Ultra-large core birefringent $\mathrm{Yb}$-doped tapered double clad fiber for high power amplifiers. Opt. Express 2018, 26, 6581, doi:10.1364/OE.26.006581.

214. Filippov, V.; Chamorovskii, Y.; Kerttula, J.; Kholodkov, A.; Okhotnikov, O.G. 600 W power scalable single transverse mode tapered double-clad fiber laser. Opt. Express 2009, 17, 1203, doi:10.1364/OE.17.001203.

215. Filippov, V.; Kerttula, J.; Chamorovskii, Y.; Golant, K.; Okhotnikov, O.G. Highly efficient 750 W tapered double-clad ytterbium fiber laser. Opt. Express 2010, 18, 12499, doi:10.1364/OE.18.012499. 
216. An, Y.; Yang, H.; Chen, X.; Xiao, H.; Huang, L.; Ma, P.; Xi, X.; Wang, X.; Zhou, P.; Pan, Z.; et al. 4 kW single-mode laser output assisted by a double-tapered double-clad fiber. In Proceedings of the Fiber Lasers XVIII: Technology and Systems; Zervas, M.N., Jauregui-Misas, C., Eds.; SPIE, 2021; Vol. 11665, p. 2.

217. Zeng, L.; Pan, Z.; Xi, X.; Yang, H.; Ye, Y.; Huang, L.; Zhang, H.; Wang, X.; Wang, Z.; Zhou, P.; et al. 5 kW monolithic fiber amplifier employing homemade spindle-shaped ytterbium-doped fiber. Opt. Lett. 2021, 46, 1393, doi:10.1364/OL.418194.

218. Yang, B.; Zhang, H.; Shi, C.; Wang, X.; Pan, Z.; Wang, Z.; Zhou, P.; Xu, X. High power monolithic tapered ytterbium-doped fiber laser oscillator. Opt. Express 2019, 27, 7585, doi:10.1364/OE.27.007585.

219. Patokoski, K.; Rissanen, J.; Noronen, T.; Gumenyuk, R.; Chamorovskii, Y.; Filippov, V.; Toivonen, J. Single-frequency 100 ns / $05 \mathrm{~mJ}$ laser pulses from all-fiber double clad ytterbium doped tapered fiber amplifier. Opt. Express 2019, 27, 31532, doi:10.1364/OE.27.031532.

220. Kerttula, J.; Filippov, V.; Chamorovskii, Y.; Golant, K.; Okhotnikov, O.G. Actively Q-switched 16-mJ tapered double-clad ytterbium-doped fiber laser. Opt. Express 2010, 18, 18543, doi:10.1364/OE.18.018543.

221. Ustimchik, V.E.; Stoliarov, D.A.; Korobko, D.A.; Chamorovskii, Y.K.; Filippov, V.N. Passively Q-switched $0.24 \mathrm{~mJ}$ ring laser based on anisotropic tapered fiber. Results Phys. 2021, 20, 103640, doi:10.1016/j.rinp.2020.103640.

222. Bobkov, K.; Levchenko, A.; Kashaykina, T.; Aleshkina, S.; Bubnov, M.; Lipatov, D.; Laptev, A.; Guryanov, A.; Leventoux, Y.; Granger, G.; et al. Scaling of average power in sub-MW peak power Yb-doped tapered fiber picosecond pulse amplifiers. Opt. Express 2021, 29, 1722, doi:10.1364/OE.413528.

223. Petrov, A.; Odnoblyudov, M.; Gumenyuk, R.; Minyonok, L.; Chumachenko, A.; Filippov, V. Picosecond Yb-doped tapered fiber laser system with 1.26 MW peak power and $200 \mathrm{~W}$ average output power. Sci. Rep. 2020, 10, 17781, doi:10.1038/s41598020-74895-z.

224. Andrianov, A. V.; Kim, A. V.; Anashkina, E.A.; Meyerov, I.B.; Lebedev, S.A.; Sergeev, A.M.; Koenig, K.; Mourou, G. Modeling of coherent beam combining from multimillijoule chirped pulse tapered fiber amplifiers. Eur. Phys. J. Spec. Top. 2015, 224, 2579-2583, doi:10.1140/epjst/e2015-02568-6.

225. Bruesselbach, H.; Jones, D.C.; Mangir, M.S.; Minden, M.; Rogers, J.L. Self-organized coherence in fiber laser arrays. Opt. Lett. 2005, 30, 1339, doi:10.1364/OL.30.001339.

226. Wang, B.; Mies, E.; Minden, M.; Sanchez, A. All-fiber $50 \mathrm{~W}$ coherently combined passive laser array. Opt. Lett. 2009, 34, 863, doi:10.1364/OL.34.000863.

227. Wang, B.; Sanchez, A. All-fiber Passive Coherent Beam Combining of Fiber Lasers and Challenges. In Proceedings of the Lasers, Sources, and Related Photonic Devices; OSA: Washington, D.C., 2012; p. FTh3A.2.

228. Wu, T.; Chang, W.; Galvanauskas, A.; Winful, H.G. Dynamical, bidirectional model for coherent beam combining in passive fiber laser arrays. Opt. Express 2010, 18, 25873, doi:10.1364/OE.18.025873.

229. Jeux, F.; Desfarges-Berthelemot, A.; Kermène, V.; Barthelemy, A. Experimental demonstration of passive coherent combining of fiber lasers by phase contrast filtering. Opt. Express 2012, 20, 28941, doi:10.1364/OE.20.028941.

230. Napartovich, A.P.; Vysotsky, D. V. Phase-locking of multicore fibre laser due to talbot self-reproduction. J. Mod. Opt. 2003, 50, 2715-2725, doi:10.1080/09500340308234581.

231. Huo, Y.; Cheo, P.K.; King, G.G. Fundamental mode operation of a 19-core phase-locked Yb-doped fiber amplifier. Opt. Express 2004, 12, 6230, doi:10.1364/opex.12.006230.

232. He, B.; Lou, Q.; Wang, W.; Zhou, J.; Zheng, Y.; Dong, J.; Wei, Y.; Chen, W. Experimental demonstration of phase locking of a two-dimensional fiber laser array using a self-imaging resonator. Appl. Phys. Lett. 2008, 92, 251115, doi:10.1063/1.2952508.

233. Corcoran, C.J.; Durville, F. Experimental demonstration of a phase-locked laser array using a self-Fourier cavity. Appl. Phys. Lett. 2005, 86, 201118, doi:10.1063/1.1925310.

234. Kong, H.J.; Yoon, J.W.; Shin, J.S.; Beak, D.H. Long-term stabilized two-beam combination laser amplifier with stimulated 
Brillouin scattering mirrors. Appl. Phys. Lett. 2008, 92, 021120, doi:10.1063/1.2831659.

235. Zhou, P.; Liu, Z.; Wang, X.; Ma, Y.; Ma, H.; Xu, X.; Guo, S. Coherent Beam Combining of Fiber Amplifiers Using Stochastic Parallel Gradient Descent Algorithm and Its Application. IEEE J. Sel. Top. Quantum Electron. 2009, 15, 248-256, doi:10.1109/JSTQE.2008.2010231.

236. Yang, Y.; Hu, M.; He, B.; Zhou, J.; Liu, H.; Dai, S.; Wei, Y.; Lou, Q. Passive coherent beam combining of four Yb-doped fiber amplifier chains with injection-locked seed source. Opt. Lett. 2013, 38, 854, doi:10.1364/OL.38.000854.

237. Fridman, M.; Nixon, M.; Davidson, N.; Friesem, A.A. Passive phase locking of 25 fiber lasers. Opt. Lett. 2010, 35, 1434, doi:10.1364/OL.35.001434.

238. Cao, J.; Hou, J.; Lu, Q.; Xu, X. Numerical research on self-organized coherent fiber laser arrays with circulating field theory. J. Opt. Soc. Am. B 2008, 25, 1187, doi:10.1364/JOSAB.25.001187.

239. Chang, W.-Z.; Wu, T.-W.; Winful, H.; Galvanauskas, A. Array size scalability of passively coherently phased fiber laser arrays. In Proceedings of the Conference on Lasers and Electro-Optics/International Quantum Electronics Conference; OSA: Washington, D.C., 2009; Vol. 18, p. JTuD63.

240. Bruesselbach, H.; Minden, M.; Rogers, J.L.; Jones, D.C.; Mangir, M.S. 200 W self-organized coherent fiber arrays. Conf. Lasers Electro-Optics/Quantum Electron. Laser Sci. Photonic Appl. Syst. Technol. 2005, paper CMDD, 532-534.

241. Loftus, T.H.; Thomas, A.M.; Norsen, M.; Minelly, J.D.; Jones, P.; Honea, E.; Shakir, S.A.; Hendow, S.; Culver, W.; Nelson, B.; et al. Four-Channel, High Power, Passively Phase Locked Fiber Array. In Proceedings of the Advanced Solid-State Photonics; OSA: Washington, D.C., 2008; p. WA4.

242. Jeux, F.; Desfarges-Berthelemot, A.; Kermene, V.; Barthelemy, A. Passive coherent combining of 15 fiber lasers by phase contrast filtering. In Proceedings of the 2013 Conference on Lasers \& Electro-Optics Europe \& International Quantum Electronics Conference CLEO EUROPE/IQEC; IEEE, 2013; pp. 1-1.

243. Zaouter, Y.; Daniault, L.; Hanna, M.; Papadopoulos, D.N.; Morin, F.; Hönninger, C.; Druon, F.; Mottay, E.; Georges, P. 2 GW peak power ultrafast fiber system using passive coherent beam combining. In Proceedings of the Lasers, Sources, and Related Photonic Devices; OSA: Washington, D.C., 2012; p. AM6A.1.

244. Daniault, L.; Hanna, M.; Papadopoulos, D.N.; Zaouter, Y.; Mottay, E.; Druon, F.; Georges, P. Coherent combining of two femtosecond chirped-pulse amplifiers in a passive architecture. In Proceedings of the Lasers, Sources, and Related Photonic Devices; OSA: Washington, D.C., 2012; p. AT4A.29.

245. Zaouter, Y.; Daniault, L.; Hanna, M.; Papadopoulos, D.N.; Morin, F.; Hönninger, C.; Druon, F.; Mottay, E.; Georges, P. Passive coherent combination of two ultrafast rod type fiber chirped pulse amplifiers. Opt. Lett. 2012, 37, 1460, doi:10.1364/OL.37.001460.

246. Bai, G.; Shen, H.; Liu, M.; Liu, K.; Zhang, H.; Niu, X.; Yang, Y.; He, B.; Zhou, J. Single-aperture passive coherent beam combining of fiber lasers based on diffractive optical element. In Proceedings of the Conference on Lasers and Electro-Optics; OSA: Washington, D.C., 2019; p. JW2A.95.

247. Tilseth, E.; Kunkel, W.M.; Leger, J.R. Demonstration of Spatial Mode Selection in a Coherently Combined Fiber Laser. In Proceedings of the 2019 Conference on Lasers and Electro-Optics Europe \& European Quantum Electronics Conference (CLEO/Europe-EQEC); IEEE, 2019; pp. 1-1.

248. Liu, H.; He, B.; Zhou, J.; Yang, Y.; Zheng, Y.; Hu, M.; Lou, Q. Experiments and Perturbative Analysis of Dammann-GratingBased Aperture Filling in a Passive Coherent Beam Combination. J. Light. Technol. 2014, 32, 2220-2227, doi:10.1109/JLT.2014.2322863.

249. Yang, Y.; Zheng, Y.; Hu, M.; Zhao, C.; Chen, X.; Liu, K.; He, B.; Zhou, J. Coherent Beam Combining of Fiber Amplifiers by means of Dammann Grating Spatial Filter. In Proceedings of the Advanced Solid State Lasers; OSA: Washington, D.C., 2014; p. AM5A.51. 
250. Jain, A.; Spiegelberg, C.; Smirnov, V.; Bochove, E.; Glebov, L. Efficient Coherent Beam Combining of Fiber Lasers Using Multiplexed Volume Bragg Gratings. In Proceedings of the Conference on Lasers and Electro-Optics 2012; OSA: Washington, D.C., 2012; p. CF2N.8.

251. Bochove, E.J.; Shakir, S.A. Analysis of a Spatial-Filtering Passive Fiber Laser Beam Combining System. IEEE J. Sel. Top. Quantum Electron. 2009, 15, 320-327, doi:10.1109/JSTQE.2008.2011999.

252. Shakir, S.A.; Culver, B.; Nelson, B.; Starcher, Y.; Bates, G.M.; Hedrick, Jr., J.W. Power scaling of passively phased fiber amplifier arrays. In Proceedings of the Optical Technologies for Arming, Safing, Fuzing, and Firing IV; Dickey, F.M., Beyer, R.A., Eds.; SPIE, 2008; Vol. 7070, p. 70700N.

253. Li, L.; Schülzgen, A.; Chen, S.; Temyanko, V.L.; Moloney, J. V.; Peyghambarian, N. Phase locking and in-phase supermode selection in monolithic multicore fiber lasers. Opt. Lett. 2006, 31, 2577, doi:10.1364/ol.31.002577.

254. Steinhausser, B.; Brignon, A.; Lallier, E.; Huignard, J.P.; Georges, P. High energy, single-mode, narrow-linewidth fiber laser source using stimulated Brillouin scattering beam cleanup. Opt. Express 2007, 15, 6464, doi:10.1364/OE.15.006464.

255. Chiang, H.-S.; Leger, J.R.; Nilsson, J.; Sahu, J. Passive Coherent Beam Combining of Fiber Lasers: Accurate Measurements of Phase Error Tolerance. In Proceedings of the CLEO: 2013; OSA: Washington, D.C., 2013; p. CW3M.6.

256. Wu, T.; Chang, W.; Galvanauskas, A.; Winful, H.G. Model for passive coherent beam combining in fiber laser arrays. Opt. Express 2009, 17, 19509, doi:10.1364/OE.17.019509.

257. Wang, B.; Mies, E.; Minden, M.; Sanchez, A. All-fiber 50 W coherently combined passive laser array. Opt. Lett. 2009, 34, 863, doi:10.1364/OL.34.000863.

258. Bloom, G.; Larat, C.; Lallier, E.; Lehoucq, G.; Bansropun, S.; Lee-Bouhours, M.-S.L.; Loiseaux, B.; Carras, M.; Marcadet, X.; Lucas-Leclin, G.; et al. Passive coherent beam combining of quantum-cascade lasers with a Dammann grating. Opt. Lett. 2011, 36, 3810, doi:10.1364/OL.36.003810.

259. Corcoran, C.J.; Durville, F. Passive Phasing in a Coherent Laser Array. IEEE J. Sel. Top. Quantum Electron. 2009, 15, 294-300, doi:10.1109/JSTQE.2008.2011494.

260. Bloom, G.; Larat, C.; Lallier, E.; Carras, M.; Marcadet, X. Coherent combining of two quantum-cascade lasers in a Michelson cavity. Opt. Lett. 2010, 35, 1917, doi:10.1364/OL.35.001917.

261. Zhao, P.; Dong, Z.; Zhang, J.; Lin, X. Passive coherent beam combination of three Nd:YAG lasers using cascaded Michelsontype compound cavities. Opt. Express 2018, 26, 18019, doi:10.1364/OE.26.018019.

262. Golubentsev, A.A.; Likhanskii, V. V.; Napartovich, A.P. Theory of phase locking of an array of lasers. In Proceedings of the Soviet Physics-JETP, American Institute of Physics; Lebedev, F. V., Napartovich, A.P., Eds.; 1987; Vol. 2109, pp. $205-218$.

263. Rockwell, D.A.; Giuliano, C.R. Coherent coupling of laser gain media using phase conjugation. Opt. Lett. 1986, 11, 147, doi:10.1364/OL.11.000147.

264. Carroll, D.L.; Johnson, R.; Pfeifer, S.J.; Moyer, R.H. Experimental investigations of stimulated Brillouin scattering beam combination. J. Opt. Soc. Am. B 1992, 9, 2214, doi:10.1364/JOSAB.9.002214.

265. Eggleston, J. Steady-state coherent Raman beam combining with multiaxial mode lasers. IEEE J. Quantum Electron. 1986, 22, 1942-1952, doi:10.1109/JQE.1986.1072899.

266. Park, S.; Cha, S.; Oh, J.; Lee, H.; Ahn, H.; Churn, K.S.; Kong, H.J. Coherent beam combination using self-phase locked stimulated Brillouin scattering phase conjugate mirrors with a rotating wedge for high power laser generation. Opt. Express 2016, 24, 8641, doi:10.1364/OE.24.008641.

267. Botez, D. Monolithic phase-locked semiconductor laser arrays. In Diode Laser Arrays; Cambridge University Press, 1994; pp. $1-71$.

268. Bochove, E.J.; Cheo, P.K.; King, G.G. Self-organization in a multicore fiber laser array. Opt. Lett. 2003, 28, 1200, doi:10.1364/OL.28.001200. 
269. Cheo, P.K.; Liu, A.; King, G.G. A high-brightness laser beam from a phase-locked multicore Yb-doped fiber laser array. IEEE Photonics Technol. Lett. 2001, 13, 439-441, doi:10.1109/68.920744.

270. Cheo, P.K. Clad pumped, eye-safe and multi-core phase-locked fiber lasers. U.S. Pat. 6 031 850, Feb. $29,2000$.

271. Minden, M.L.; Bruesselbach, H.W.; Rogers, J.L.; Mangir, M.S.; Jones, D.C.; Dunning, G.J.; Hammon, D.L.; Solis, A.J.; Vaughan, L. Self-organized coherence in fiber laser arrays. In Proceedings of the Fiber Lasers: Technology, Systems, and Applications; Durvasula, L.N., Ed.; 2004; Vol. 5335, p. 89.

272. Zhou, P.; Chen, Z.; Xu, X.; Hou, J.; Liu, Z. Modeling self-organized coherent fiber laser array. In Proceedings of the HighPower Lasers and Applications IV; Fan, D., Walter, R.F., Eds.; 2007; Vol. 6823, p. 68230G.

273. Chen, Z.; Hou, J.; Zhou, P.; Wang, X.; Xu, X.; Jiang, Z.; Liu, Z. Mutual injection locking and coherent combining of three individual fiber lasers. Opt. Commun. 2009, 282, 60-63, doi:10.1016/j.optcom.2008.09.037.

274. Chen, Z.; Hou, J.; Wang, Z.; Chen, S. Self-organized coherence in fiber laser arrays by mutual injection locking. In Proceedings of the Laser Optics 2010; Rosanov, N.N., Venediktov, V.Y., Eds.; 2010; Vol. 7822, p. 782203.

275. Hansch, T.W.; Couillaud, B. Laser frequency stabilization by polarization spectroscopy of a reflecting reference cavity. Opt. Commun. 1980, 35, 441-444, doi:10.1016/0030-4018(80)90069-3.

276. Anderegg, J.; Brosnan, S.; Cheung, E.; Epp, P.; Hammons, D.; Komine, H.; Weber, M.; Wickham, M. Coherently coupled highpower fiber arrays. In Proceedings of the LEOS Summer Topical Meeting; Brown, A.J.W., Nilsson, J., Harter, D.J., Tünnermann, A., Eds.; 2006; Vol. 6102, p. 61020U.

277. O'Meara, T.R. The multidither principle in adaptive optics. J. Opt. Soc. Am. 1977, 67, 306, doi:10.1364/JOSA.67.000306.

278. Ma, Y.; Zhou, P.; Wang, X.; Ma, H.; Xu, X.; Si, L.; Liu, Z.; Zhao, Y. Coherent beam combination with single frequency dithering technique. Opt. Lett. 2010, 35, 1308, doi:10.1364/OL.35.001308.

279. Ma, Y.; Wang, X.; Leng, J.; Xiao, H.; Dong, X.; Zhu, J.; Du, W.; Zhou, P.; Xu, X.; Si, L.; et al. Coherent beam combination of $1.08 \mathrm{~kW}$ fiber amplifier array using single frequency dithering technique. Opt. Lett. 2011, 36, 951, doi:10.1364/OL.36.000951.

280. Ma, P.F.; Zhou, P.; Su, R.T.; Ma, Y.X.; Liu, Z.J. Coherent polarization beam combining of eight fiber lasers using singlefrequency dithering technique. Laser Phys. Lett. 2012, 9, 456-458, doi:10.7452/lapl.201210016.

281. Tang, X.; Huang, Z.; Zhang, D.; Wang, X.; Li, J.; Liu, C. An active phase locking of multiple fiber channels via square wave dithering algorithm. Opt. Commun. 2014, 321, 198-204, doi:10.1016/j.optcom.2014.01.076.

282. Shay, T.M.; Benham, V.; Baker, J.T.; Sanchez, A.D.; Pilkington, D.; Lu, C.A. Self-Synchronous and Self-Referenced Coherent Beam Combination for Large Optical Arrays. IEEE J. Sel. Top. Quantum Electron. 2007, 13, 480-486, doi:10.1109/JSTQE.2007.897173.

283. Shay, T.M. Theory of electronically phased coherent beam combination without a reference beam. Opt. Express 2006, 14, 12188, doi:10.1364/OE.14.012188.

284. Daniault, L.; Hanna, M.; Lombard, L.; Zaouter, Y.; Mottay, E.; Goular, D.; Bourdon, P.; Druon, F.; Georges, P. Coherent beam combining of two femtosecond fiber chirped-pulse amplifiers. Opt. Lett. 2011, 36, 621, doi:10.1364/OL.36.000621.

285. Wagner, T.J. Fiber laser beam combining and power scaling progress: Air Force Research Laboratory Laser Division. In Proceedings of the Fiber Lasers IX: Technology, Systems, and Applications; 2012; Vol. 8237, p. 823718.

286. Bourdon, P.; Jacqmin, H.; Augère, B.; Durécu, A.; Goular, D.; Rouzé, B.; Domel, R.; Fleury, D.; Planchat, C.; Lombard, L. Target-in-the-loop frequency-tagging coherent combining of 7 fiber lasers up to $1 \mathrm{~km}$ range. In Proceedings of the Fiber Lasers XVIII: Technology and Systems; Zervas, M.N., Jauregui-Misas, C., Eds.; SPIE, 2021; p. 50.

287. Kabeya, D.; Kermene, V.; Fabert, M.; Benoist, J.; Desfarges-Berthelemot, A.; Barthelemy, A. Active coherent combining of laser beam arrays by means of phase-intensity mapping in an optimization loop. Opt. Express 2015, 23, 31059, doi:10.1364/OE.23.031059.

288. Wang, D.; Du, Q.; Zhou, T.; Li, D.; Wilcox, R. Stabilization of the 81-channel coherent beam combination using machine 
learning. Opt. Express 2021, 29, 5694, doi:10.1364/OE.414985.

289. Du, Q.; Wang, D.; Zhou, T.; Li, D.; Wilcox, R. 81-beam coherent combination using a programmable array generator. Opt. Express 2021, 29, 5407, doi:10.1364/OE.416499.

290. Su, R.; Zhou, P.; Wang, X.; Zhang, H.; Xu, X. Impact of temporal and spectral aberrations on coherent beam combination of nanosecond fiber lasers. Appl. Opt. 2013, 52, 2187, doi:10.1364/AO.52.002187.

291. Yu, H.L.; Ma, P.F.; Wang, X.L.; Su, R.T.; Zhou, P.; Chen, J.B. Influence of temporal-spectral effects on ultrafast fiber coherent polarization beam combining system. Laser Phys. Lett. 2015, 12, 105301, doi:10.1088/1612-2011/12/10/105301.

292. Ma, P.; Tao, R.; Wang, X.; Ma, Y.; Su, R.; Zhou, P. Coherent polarization beam combination of four mode-locked fiber MOPAs in picosecond regime. Opt. Express 2014, 22, 4123, doi:10.1364/OE.22.004123.

293. Yu, C.X.; Kansky, J.E.; Shaw, S.E.J.; Murphy, D.V.; Higgs, C. Coherent beam combining of large number of PM fibres in 2-D fibre array. Electron. Lett. 2006, 42, 1024, doi:10.1049/el:20061938.

294. Lombard, L.; Azarian, A.; Cadoret, K.; Bourdon, P.; Goular, D.; Canat, G.; Jolivet, V.; Jaouën, Y.; Vasseur, O. Coherent beam combination of narrow-linewidth $15 \mu \mathrm{m}$ fiber amplifiers in a long-pulse regime. Opt. Lett. 2011, 36, 523, doi:10.1364/OL.36.000523.

295. Siiman, L.A.; Chang, W.; Zhou, T.; Galvanauskas, A. Coherent femtosecond pulse combining of multiple parallel chirped pulse fiber amplifiers. Opt. Express 2012, 20, 18097, doi:10.1364/OE.20.018097.

296. Müller, M.; Klenke, A.; Steinkopff, A.; Stark, H.; Tünnermann, A.; Limpert, J. 3.5 kW coherently combined ultrafast fiber laser. Opt. Lett. 2018, 43, 6037, doi:10.1364/OL.43.006037.

297. Zhou, S.; Wise, F.W.; Ouzounov, D.G. Divided-pulse amplification of ultrashort pulses. Opt. Lett. 2007, 32, 871, doi:10.1364/OL.32.000871.

298. Stark, H.; Müller, M.; Kienel, M.; Klenke, A.; Limpert, J.; Tünnermann, A. Electro-optically controlled divided-pulse amplification. Opt. Express 2017, 25, 13494, doi:10.1364/OE.25.013494.

299. Guichard, F.; Lavenu, L.; Hanna, M.; Zaouter, Y.; Georges, P. Coherent combining efficiency in strongly saturated dividedpulse amplification systems. Opt. Express 2016, 24, 25329, doi:10.1364/OE.24.025329.

300. Zaouter, Y.; Guichard, F.; Daniault, L.; Hanna, M.; Morin, F.; Hönninger, C.; Mottay, E.; Druon, F.; Georges, P. Femtosecond fiber chirped- and divided-pulse amplification system. Opt. Lett. 2013, 38, 106, doi:10.1364/OL.38.000106.

301. Kienel, M.; Klenke, A.; Eidam, T.; Hädrich, S.; Limpert, J.; Tünnermann, A. Energy scaling of femtosecond amplifiers using actively controlled divided-pulse amplification. Opt. Lett. 2014, 39, 1049, doi:10.1364/OL.39.001049.

302. Guichard, F.; Zaouter, Y.; Hanna, M.; Mai, K.-L.; Morin, F.; Hönninger, C.; Mottay, E.; Georges, P. High-energy chirped- and divided-pulse Sagnac femtosecond fiber amplifier. Opt. Lett. 2015, 40, 89, doi:10.1364/OL.40.000089.

303. Daniault, L.; Hanna, M.; Papadopoulos, D.N.; Zaouter, Y.; Mottay, E.; Druon, F.; Georges, P. High peak-power stretcher-free femtosecond fiber amplifier using passive spatio-temporal coherent combining. Opt. Express 2012, 20, 21627, doi:10.1364/OE.20.021627.

304. Kienel, M.; Müller, M.; Klenke, A.; Eidam, T.; Limpert, J.; Tünnermann, A. Multidimensional coherent pulse addition of ultrashort laser pulses. Opt. Lett. 2015, 40, 522, doi:10.1364/OL.40.000522.

305. Loftus, T.H.; Liu, A.; Hoffman, P.R.; Thomas, A.M.; Norsen, M.; Royse, R.; Honea, E. 522 W average power, spectrally beamcombined fiber laser with near-diffraction-limited beam quality. Opt. Lett. 2007, 32, 349, doi:10.1364/OL.32.000349.

306. Andrusyak, O.; Smirnov, V.; Venus, G.; Vorobiev, N.; Glebov, L. Applications of volume Bragg gratings for spectral control and beam combining of high power fiber lasers. In Proceedings of the Fiber Lasers VI: Technology, Systems, and Applications; Gapontsev, D. V., Kliner, D.A., Dawson, J.W., Tankala, K., Eds.; 2009; Vol. 7195, p. 71951Q.

307. Regelskis, K.; Hou, K.-C.; Raciukaitis, G.; Galvanauskas, A. Spatial-dispersion-free spectral beam combining of high power pulsed Yb-doped fiber lasers. In Proceedings of the 2008 Conference on Lasers and Electro-Optics; IEEE, 2008; pp. 1-2. 
308. Chang, W.-Z.; Zhou, T.; Siiman, L.A.; Galvanauskas, A. Femtosecond pulse spectral synthesis in coherently-spectrally combined multi-channel fiber chirped pulse amplifiers. Opt. Express 2013, 21, 3897, doi:10.1364/OE.21.003897.

309. Guichard, F.; Hanna, M.; Lombard, L.; Zaouter, Y.; Hönninger, C.; Morin, F.; Druon, F.; Mottay, E.; Georges, P. Two-channel pulse synthesis to overcome gain narrowing in femtosecond fiber amplifiers. Opt. Lett. 2013, 38, 5430, doi:10.1364/OL.38.005430.

310. Rigaud, P.; Kermene, V.; Bouwmans, G.; Bigot, L.; Desfarges-Berthelemot, A.; Labat, D.; Le Rouge, A.; Mansuryan, T.; Barthélémy, A. Spatially dispersive amplification in a 12-core fiber and femtosecond pulse synthesis by coherent spectral combining. Opt. Express 2013, 21, 13555, doi:10.1364/OE.21.013555.

311. Guichard, F.; Hanna, M.; Lombard, L.; Zaouter, Y.; Hönninger, C.; Morin, F.; Druon, F.; Mottay, E.; Georges, P. Spectral pulse synthesis in large-scale ultrafast coherent combining systems. Eur. Phys. J. Spec. Top. 2015, 224, 2545-2549, doi:10.1140/epjst/e2015-02563-y.

312. Weyrauch, T.; Vorontsov, M.A.; Carhart, G.W.; Beresnev, L.A.; Rostov, A.P.; Polnau, E.E.; Liu, J.J. Experimental demonstration of coherent beam combining over a $7 \mathrm{~km}$ propagation path. Opt. Lett. 2011, 36, 4455, doi:10.1364/OL.36.004455.

313. Goodno, G.D.; McNaught, S.J.; Rothenberg, J.E.; McComb, T.S.; Thielen, P.A.; Wickham, M.G.; Weber, M.E. Active phase and polarization locking of a $14 \mathrm{~kW}$ fiber amplifier. Opt. Lett. 2010, 35, 1542, doi:10.1364/OL.35.001542.

314. Prieto, C.; Vaamonde, E.; Diego-Vallejo, D.; Jimenez, J.; Urbach, B.; Vidne, Y.; Shekel, E. Dynamic laser beam shaping for laser aluminium welding in e-mobility applications. Procedia CIRP 2020, 94, 596-600, doi:10.1016/j.procir.2020.09.084.

315. Montoya, J.; Hwang, C.; Martz, D.; Aleshire, C.; Fan, T.Y.; Ripin, D.J. Photonic lantern kW-class fiber amplifier. Opt. Express 2017, 25, 27543, doi:10.1364/OE.25.027543.

316. Shekel, E.; Vidne, Y.; Urbach, B. 16kW single mode CW laser with dynamic beam for material processing. In Proceedings of the Fiber Lasers XVII: Technology and Systems; Dong, L., Zervas, M.N., Eds.; SPIE, 2020; Vol. 11260, p. 73.

317. Uberna, R.; Bratcher, A.; Alley, T.G.; Sanchez, A.D.; Flores, A.S.; Pulford, B. Coherent combination of high power fiber amplifiers in a two-dimensional re-imaging waveguide. Opt. Express 2010, 18, 13547, doi:10.1364/OE.18.013547.

318. Gerstner, E. Extreme light. Nat. Mater. 2016, 15, 1-1, doi:10.1038/nmat4533. 